Supporting Information for

\title{
Prescribing silver chirality with DNA origami
}

Yinan Zhang, ${ }^{\dagger}{ }^{\dagger}$ Zhi-bei Qu ${ }^{\dagger}$ Chu Jiang, ${ }^{\S}$ Yingying Liu, ${ }^{\dagger}$ Raghu Pradeep Narayanan, ${ }^{\ddagger}$ Dewight Williams, ${ }^{\prime}$ Xiaolei Zuo, ${ }^{\perp}$ Lihua Wang, ${ }^{\# \&}$ Hao Yan, ${ }^{\ddagger}$ Huajie Liu*, ${ }^{*}$ and Chunhai Fan ${ }^{\dagger}$

${ }^{\dagger}$ School of Chemistry and Chemical Engineering, Frontiers Science Center for Transformative Molecules and National Center for Translational Medicine, Shanghai Jiao Tong University, Shanghai, China.

${ }^{\ddagger}$ Center for Molecular Design and Biomimetics, The Biodesign Institute, School of Molecular Sciences, Arizona State University, Tempe, AZ, USA.

${ }^{\S}$ School of Chemical Science and Engineering, Shanghai Research Institute for Intelligent Autonomous Systems, Key Laboratory of Advanced Civil Engineering Materials of Ministry of Education, Tongji University, Shanghai, China.

"Erying Materials Center, Office of Knowledge Enterprise Development, Arizona State University, Tempe, AZ, USA.

${ }^{\perp}$ Institute of Molecular Medicine, Shanghai Key Laboratory for Nucleic Acids Chemistry and Nanomedicine, Renji Hospital, School of Medicine, Shanghai Jiao Tong University, Shanghai, China.

\#Bioimaging Center, Shanghai Synchrotron Radiation Facility, Zhangjiang Laboratory, Shanghai Advanced Research Institute, Chinese Academy of Sciences, Shanghai, China.

${ }^{\&}$ Shanghai Key Laboratory of Green Chemistry and Chemical Processes, School of Chemistry and Molecular Engineering, East China Normal University, 500 Dongchuan Road, Shanghai, China.

*Correspondence to: liuhuajie@tongji.edu.cn 


\section{Experimental Section}

Materials. M13mp18 viral DNA was purchased from Tilibit nanosystems and used without further purification. The staple strands with PAGE purification were purchased from Jie Li Biology. Thiolated DNA strands with HPLC purification were purchased from Takara Bio. Silver nitrate was purchased from Sigma. All other chemicals were purchased from Sinopharm. Water was purified with a Millipore Milli-Q Integral water purification system (resistivity=18.2 $\mathrm{M} \Omega \cdot \mathrm{cm}$ ).

Folding DNA origami substrate. $2 \mathrm{nM} \mathrm{M} 13 \mathrm{mp} 18$ viral DNA was mixed with $20 \mathrm{nM}$ of each staple in $1 \times$ TAE buffer ( $40 \mathrm{mM}$ Tris, $20 \mathrm{mM}$ acetic acid, $2 \mathrm{mM}$ EDTA, pH 8.0) containing $12.5 \mathrm{mM} \mathrm{Mg}^{2+}$ and subjected to an annealing ramp that cooled from $85^{\circ} \mathrm{C}$ to $25^{\circ} \mathrm{C}$ in $120 \mathrm{~min}$. Excess staples were removed by centrifugation for $10 \mathrm{~min}$ at $3000 \times \mathrm{g}$ for three times with ultra-centrifugal filters (Amicon).

Assembly of DNA origami dimers and 1D filaments. For assembly of DNA origami dimers, two origami monomers with their left or right edge staples omitted respectively were mixed at a stoichiometric ratio. The edge staples engineered through interexchange of eight bases were added at ten times the concentration of monomers, followed by a 4-hour incubation at $35^{\circ} \mathrm{C}$. For assembly of $1 \mathrm{D}$ filaments, there were onepot and two-step protocols. In the former, the engineered edge staples were mixed with the other staples in DNA origami folding. In the latter, DNA origami monomers with staples of both edges omitted were mixed with the engineered edge staples and incubated overnight at $35^{\circ} \mathrm{C}$.

Guiding silver metallization with DNA origami. Ammonia solution (10\%) was added to the silver nitrate solution $(100 \mathrm{mM})$ drop by drop until the resulting turbid solution turned clear again. Subsequently, diluted nitric acid (4 M) was slowly added to maintain the $\mathrm{pH}$ at $\sim 8.5$. Varied concentrations of silver(I) precursors were fully mixed with DNA origami solution ( $2 \mathrm{nM}$ ). The mixture was incubated at RT for $1 \mathrm{~min}$, followed by rapid addition of glucose at a doubled concentration to that of precursors. The mixture was held for specified times before characterization. The optimal reaction time for silver deposition on pcDNA is $10 \mathrm{~min}$.

AFM characterization. A droplet of sample $(2 \mu \mathrm{L})$ was deposited on freshly cleaved mica surface and left to absorb for $3 \mathrm{~min}$. Then $40 \mu \mathrm{L} 1 \times \mathrm{TAE}$ buffer with $12.5 \mathrm{mM}$ $\mathrm{Mg}^{2+}$ was added to the liquid cell and a SNL-10 (Bruker, Inc.) tip was used to image the sample in PeakForce-tapping mode on a Multimode VIII AFM (Bruker, Inc.)

TEM characterization. A droplet of sample $(7 \mu \mathrm{L})$ was deposited on a glowdischarged grid and left to absorb for $10 \mathrm{~min}$. The remaining buffer was removed by a Whatman paper and a droplet of water was used to clean the grid and then wicked. TEM images were obtained with a Tecnei G2-20S TWIN system (FEI, Inc) or JEOL JEMF200, operated at $200 \mathrm{kV}$ in a bright field mode.

XPS characterization. A droplet of sample $(10 \mu \mathrm{L})$ was deposited on a glowdischarged silicon plate and left to absorb for 2 min. Excess buffer was wicked by a Whatman paper. The sample was analyzed using a Kratos Axis ULTRADLD system with an Al Ka mono X-ray source of $1486.69 \mathrm{eV}$ at a take-off angle of $54.7^{\circ}$. All the 
peaks were aligned with respect to the $\mathrm{C} 1 \mathrm{~s}$ binding energy $(\mathrm{BE})$ at $285 \mathrm{eV}$. The data was analyzed by XPSPEAK 4.1 software with Shirley background subtractions and Gaussian-Lorentzian functions for peak fittings.

UV characterization. Absorbance spectra of $100-\mu \mathrm{L}$ DNA origami solution $(6 \mathrm{nM})$ were obtained with a quartz cuvette (1-cm path length) on a UV-vis spectrometer (Shimadzu UV-2600). DNA origami was incubated with silver precursors (final concentration: $2 \mathrm{mM}$ ) for $10 \mathrm{~min}$ before measuring the absorbance of silver-DNA solution.

FTIR characterization. A droplet of sample $(10 \mu \mathrm{L})$ was deposited on a glowdischarged silicon plate and left to absorb for 2 min. Excess buffer was wicked by a Whatman paper. The sample was analyzed using a Thermo Scientific Nicolet iS10 system.

CD characterization. Circular dichroism measurements were performed with a quartz cuvette (1-cm path length) using a Chirascan CD photometer (Applied Photophysics, Inc.).

Cyro-EM characterization. A droplet of sample $(3 \mu \mathrm{L})$ was deposited on a glowdischarged ultrathin carbon film on Lacey grids. The grids were blotted for 5 seconds and then plunged into liquid ethane cooled by liquid nitrogen, using a Vitrobot Mark IV (FEI). The samples were analyzed with a Titan Krios transmission electron microscope (FEI) operated at $300 \mathrm{kV}$.

Height analysis of silver protrusion. Ratios of protruded areas of silver patterns at different heights were analyzed by Gwyddion software (http://gwyddion.net/). The theoretical ratios of protrusion were calculated following a reported literature (Ref. 41 in the main text). Distorted DNA origamis were excluded from the analysis.

DFT simulation. All $a b$ initio calculations were carried out using the Gaussian 09 program (version: Rev. E.01, 2015) and GAUSSVIEW 5.0 visualization program. Linear configuration of $\mathrm{Ag}\left(\mathrm{NH}_{3}\right)_{2}{ }^{+}$coordination complex was employed to study the interaction to bases through coordination replacements, hydrogen bonds or metal- $\pi$ interactions. Unrestricted functional B3LYP ${ }^{1}$ with LANL2DZ basis $\operatorname{set}^{2}$ was used for silver in geometric optimizations, while polarized basis sets of $6-31+G(d, p)$ were used for the $\mathrm{H}, \mathrm{C}, \mathrm{N}$, and $\mathrm{O}$ atoms ${ }^{3}$. The ground state was converged to the threshold of $10^{-4}$ Hartree/ $\AA$ on the Cartesian gradients while the energy was converged to a tight threshold of $10^{-6}$ Hartree. Frequency analyses were used to verify that the optimized structures were at their least local minimum that no imaginary frequencies were found in the final structures. Ultrafine integration grids were employed for all the calculations. The interaction energy between the nucleic bases with $\mathrm{Ag}\left(\mathrm{NH}_{3}\right)_{2}{ }^{+}$adsorption is defined as:

$$
E_{\text {abs }}=E_{\text {total }}-E_{A g(N H 3) 2}-E_{\text {base }}
$$

$E_{a b s}$ represent the absorption energy, and $E_{\text {total }}, E_{A g(N H 3) 2}, E_{\text {base }}$ represent the energy of final complex, $\mathrm{Ag}\left(\mathrm{NH}_{3}\right)_{2}{ }^{+}$, and the corresponding nucleic base, respectively. 


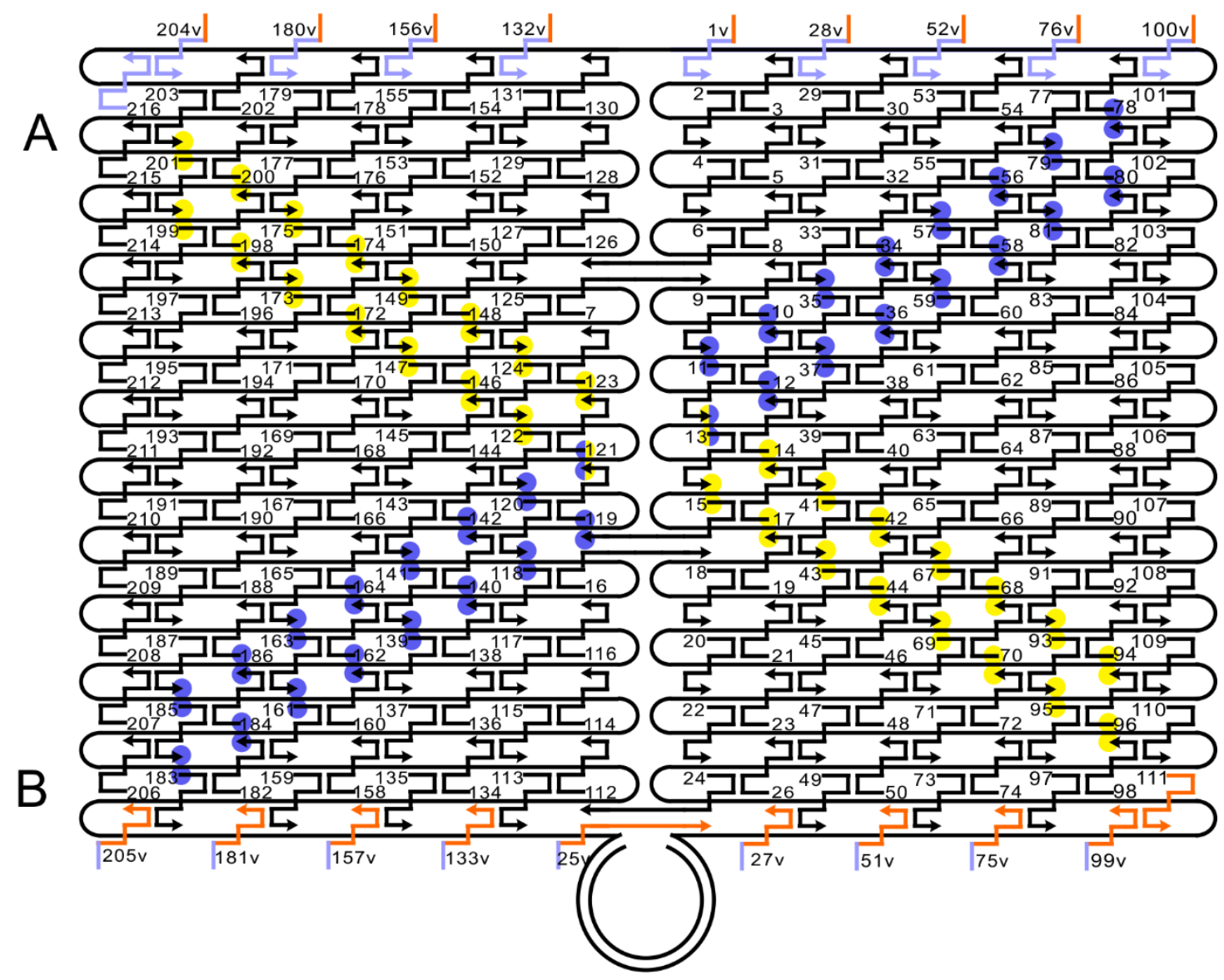

Figure S1. Schematic design of helical pcDNA templates with a pitch of $100 \mathrm{~nm}$ on DNA origami. DNA protrusions are placed along the diagonal line (A or B). Eight bases were exchanged between the staples at the bottom and top edge of DNA origami to roll the rectangle into a tube. 


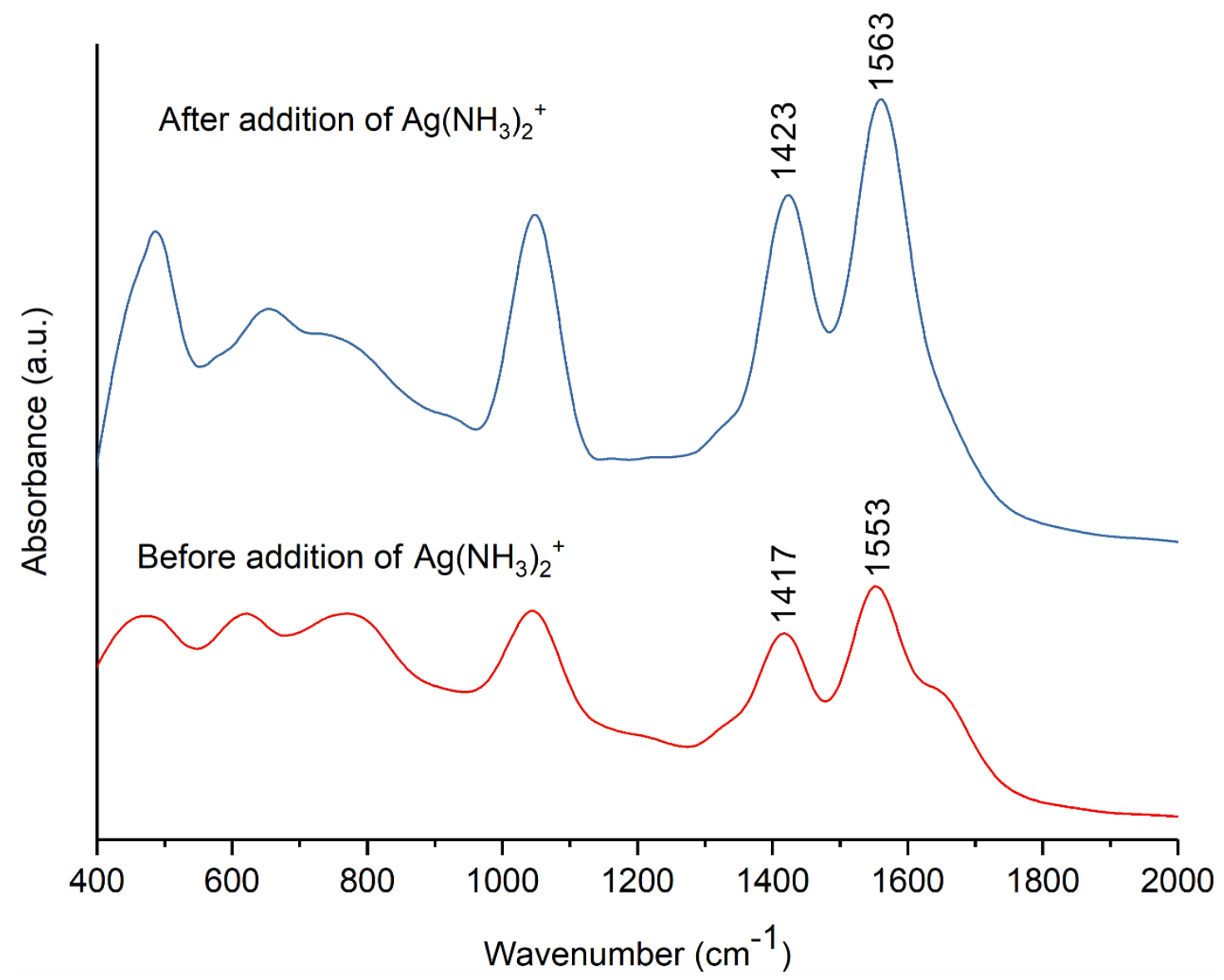

Figure S2. FTIR spectra of the DNA origami carrying pcDNA before and after addition of diamine silver(I) complexes. 


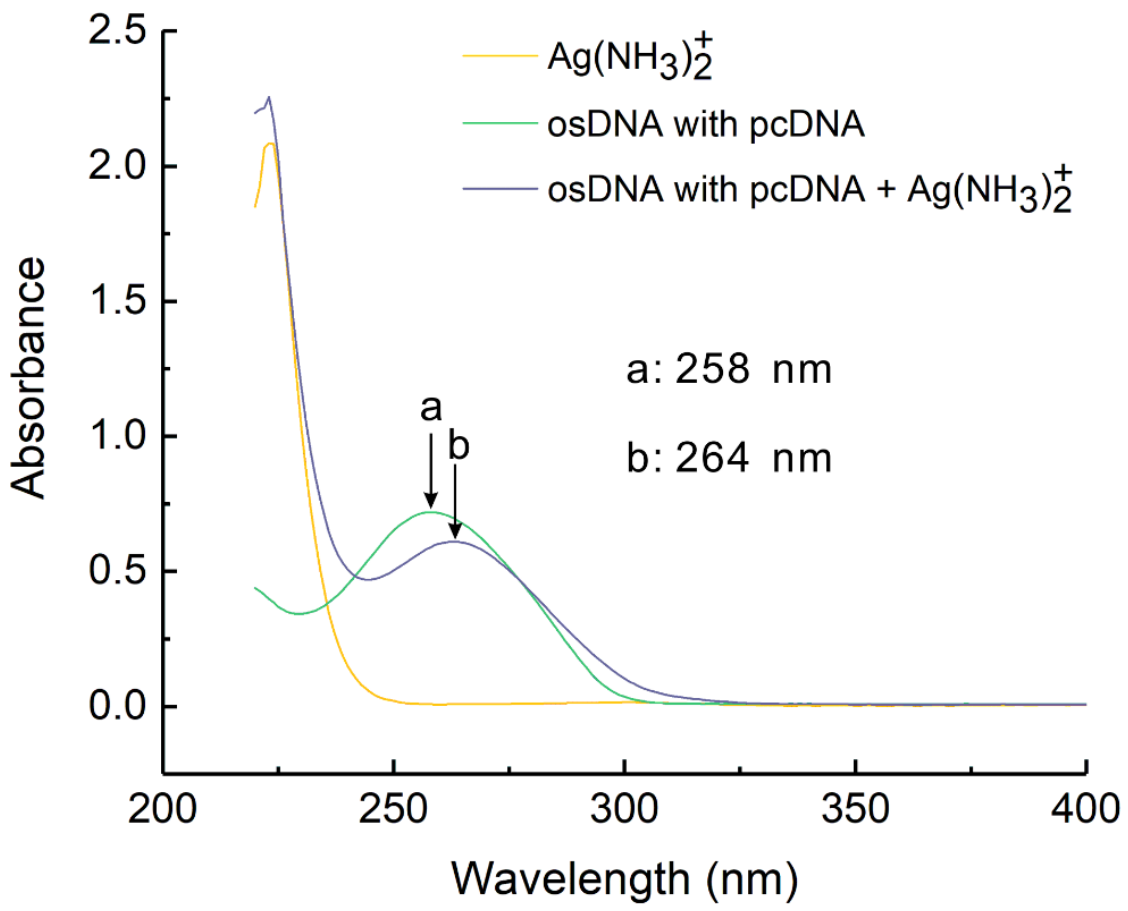

Figure S3. UV absorbance spectra of DNA origami carrying pcDNA before and after addition of diamine silver(I) complexes. 


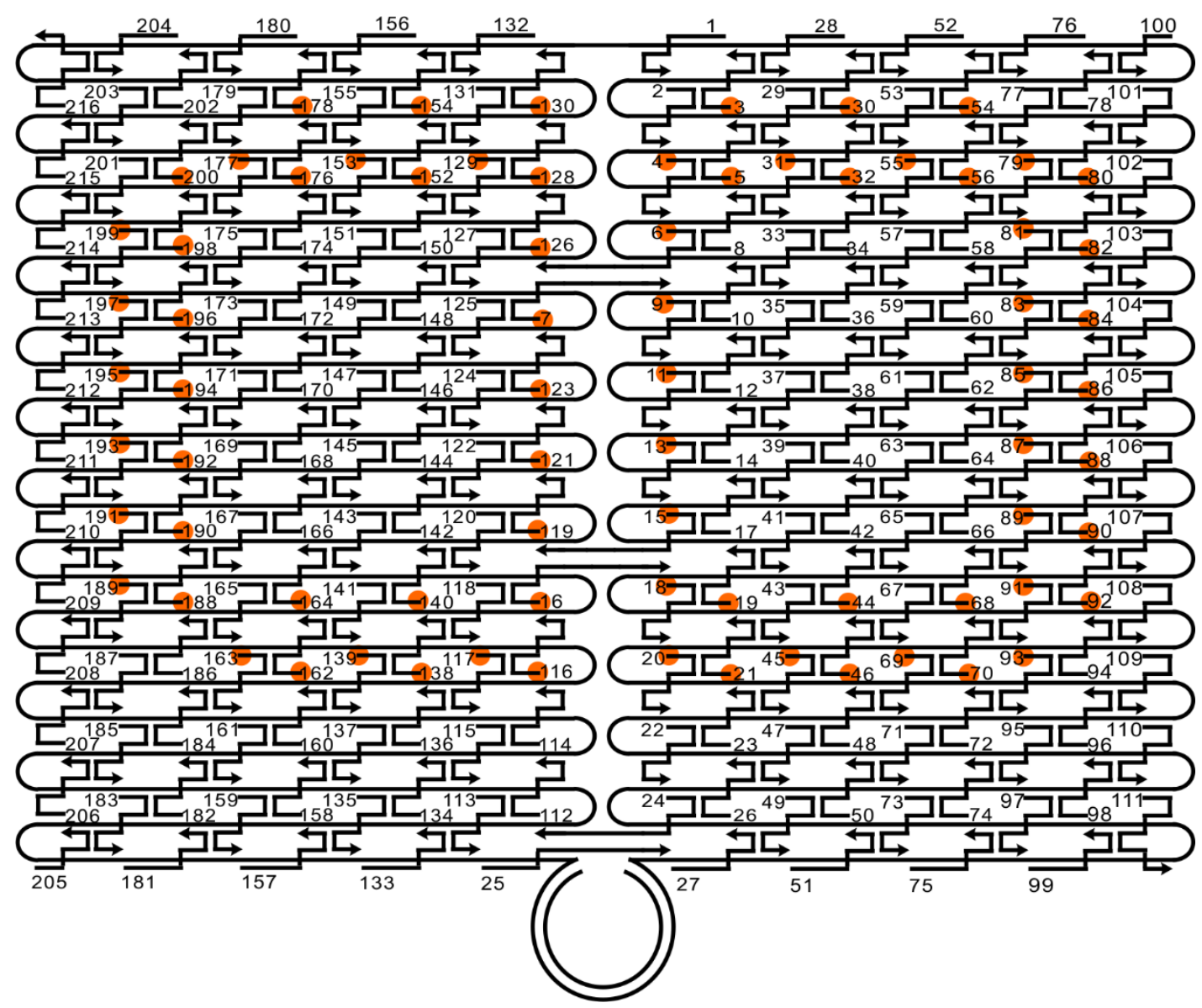

Figure S4. Schematic design of " 8 "-shaped pcDNA template on the rectangular DNA origami. DNA protrusions are denoted by orange dots. 


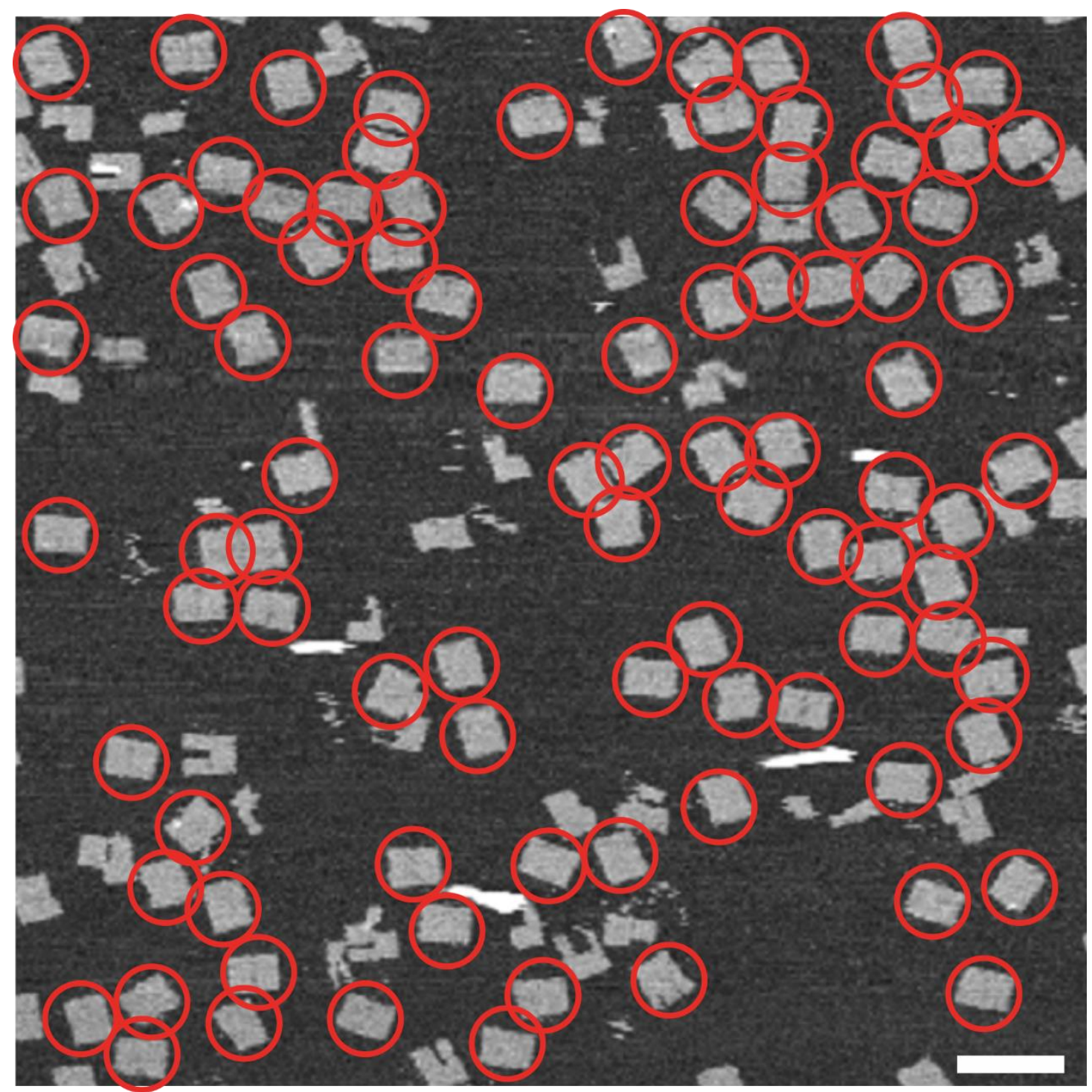

Figure S5. Silver patterns on DNA origami without silver precursors. Reaction time: 10 min. Reductant: glucose. Scale bar: $200 \mathrm{~nm}$. DNA origamis included in the statistics are circled in red. 


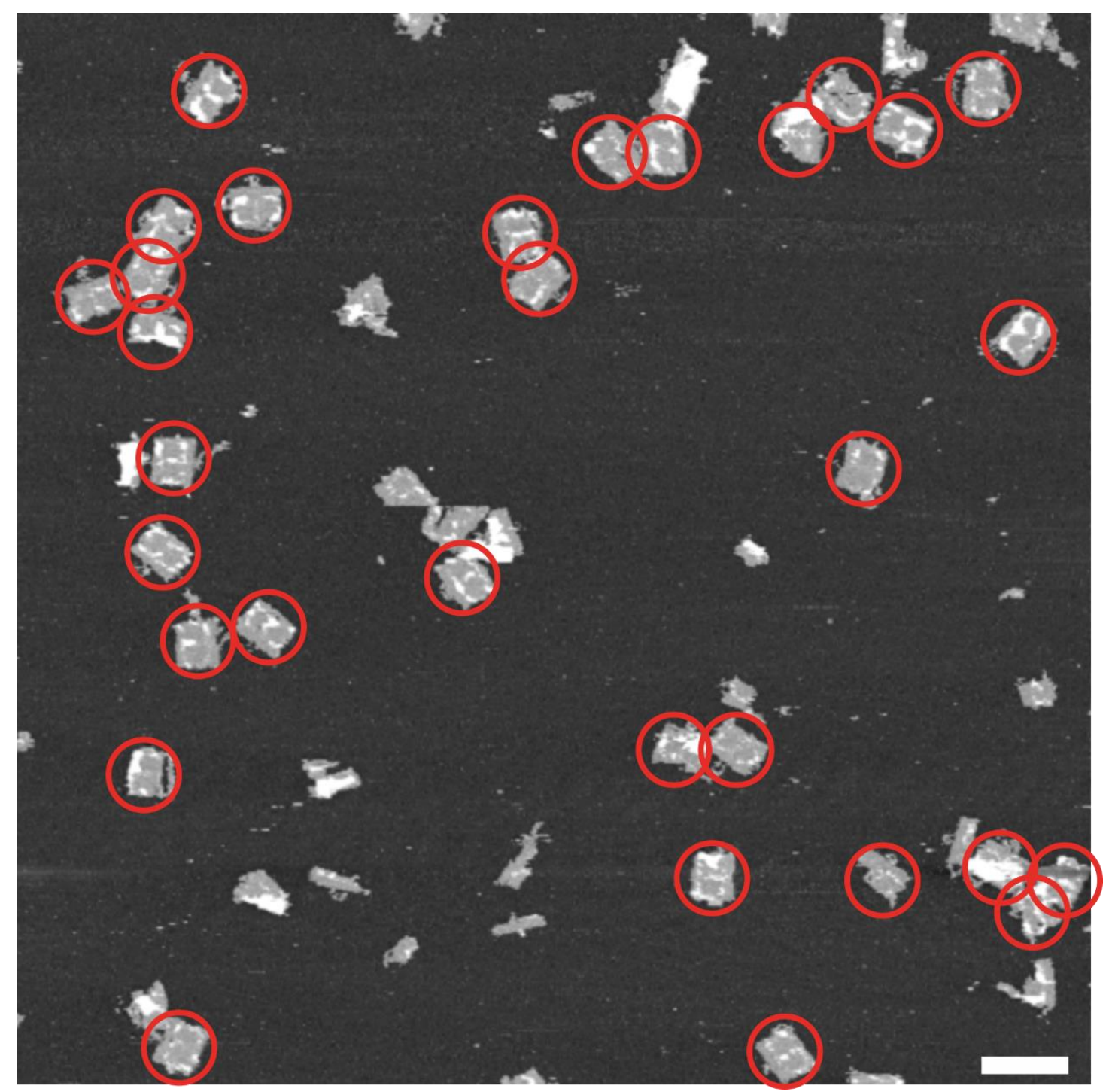

Figure S6. Silver patterns on DNA origami at $0.5 \mathrm{mM}$ silver precursors. Reaction time: 10 min. Reductant: glucose. Scale bar: $200 \mathrm{~nm}$. DNA origamis included in the statistics are circled in red. 


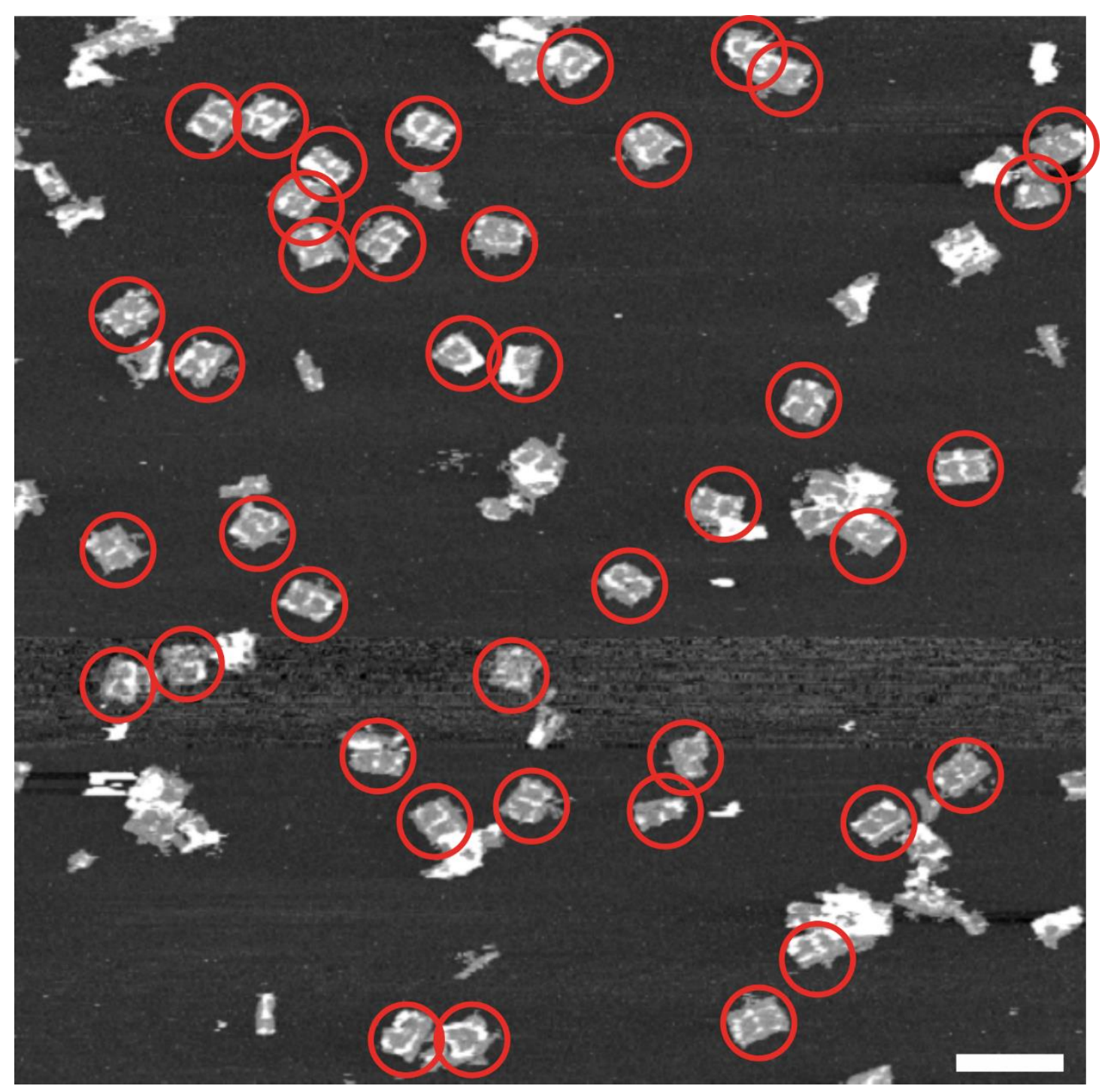

Figure S7. Silver patterns on DNA origami at $1 \mathrm{mM}$ silver precursors. Reaction time: 10 min. Reductant: glucose. Scale bar: $200 \mathrm{~nm}$. DNA origamis included in the statistics are circled in red. 


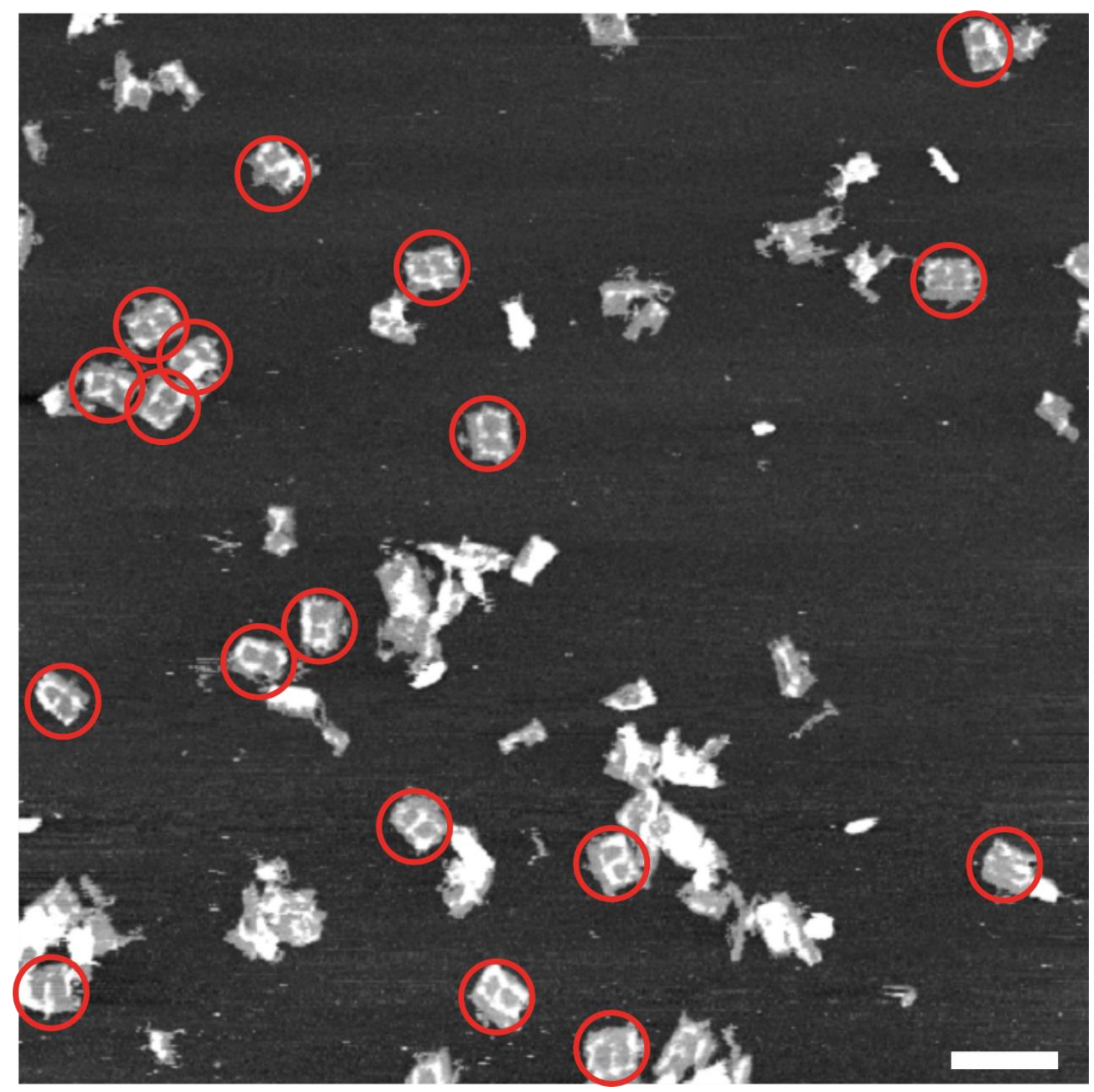

Figure S8. Silver patterns on DNA origami at $2 \mathrm{mM}$ silver precursors. Reaction time: 10 min. Reductant: glucose. Scale bar: $200 \mathrm{~nm}$. DNA origamis included in the statistics are circled in red. 


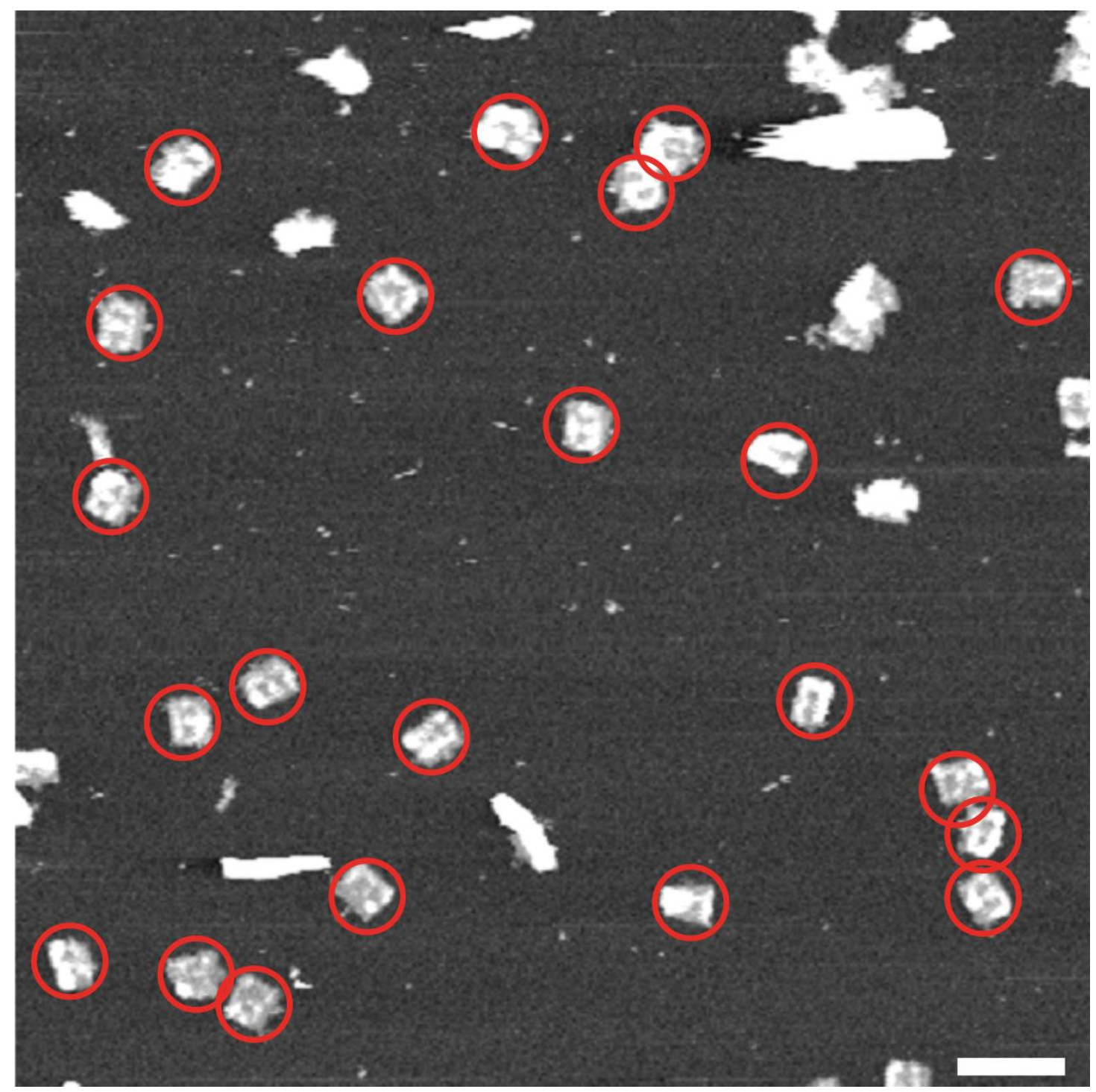

Figure S9. Silver patterns on DNA origami at $4 \mathrm{mM}$ silver precursors. Reaction time: 10 min. Reductant: glucose. Scale bar: $200 \mathrm{~nm}$. DNA origamis included in the statistics are circled in red. 


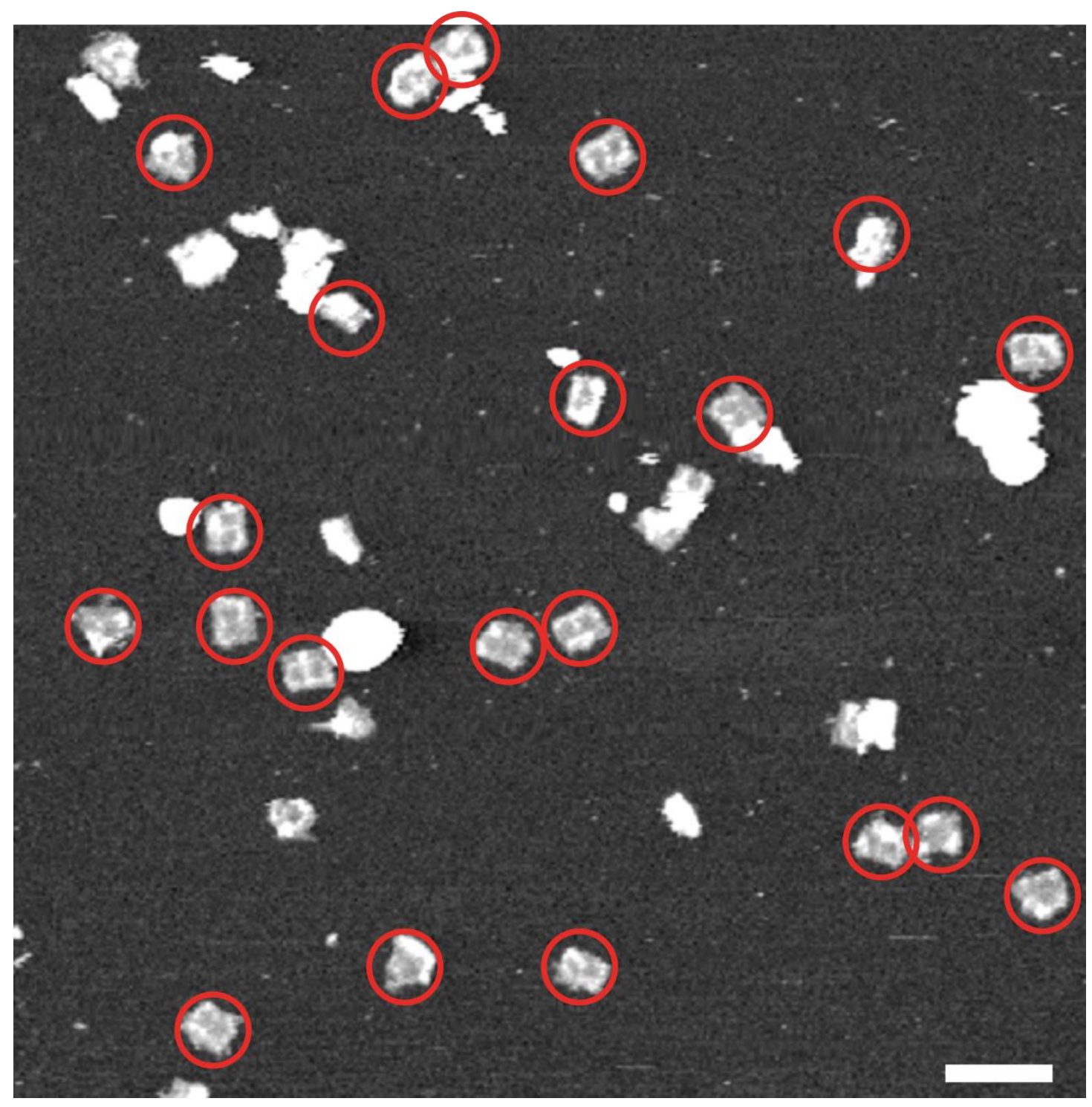

Figure S10. Silver patterns on DNA origami at $6 \mathrm{mM}$ silver precursors. Reaction time: 10 min. Reductant: glucose. Scale bar: $200 \mathrm{~nm}$. DNA origamis included in the statistics are circled in red. 


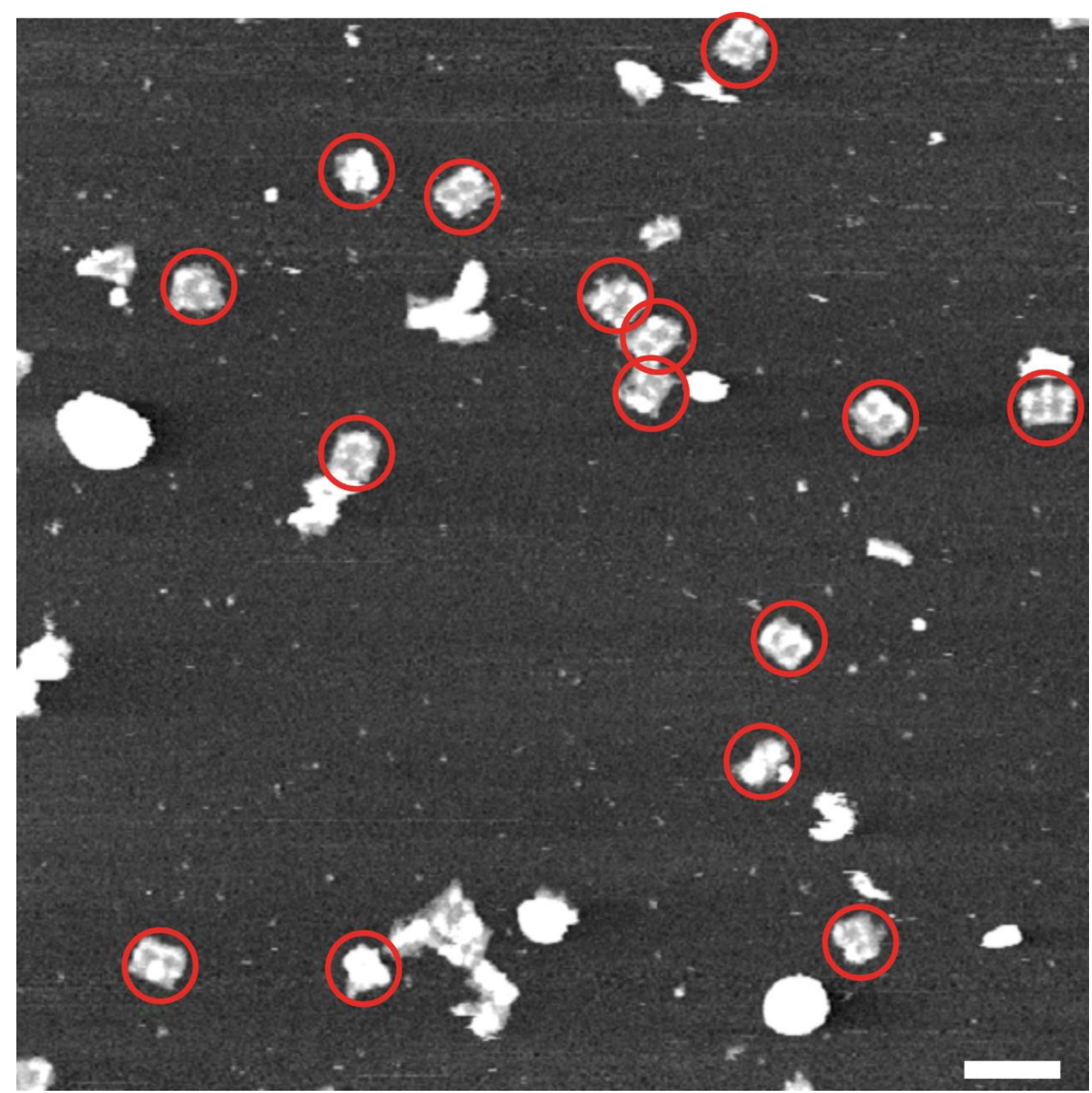

Figure S11. Silver patterns on DNA origami at $8 \mathrm{mM}$ silver precursors. Reaction time: 10 min. Reductant: glucose. Scale bar: $200 \mathrm{~nm}$. DNA origamis included in the statistics are circled in red. 


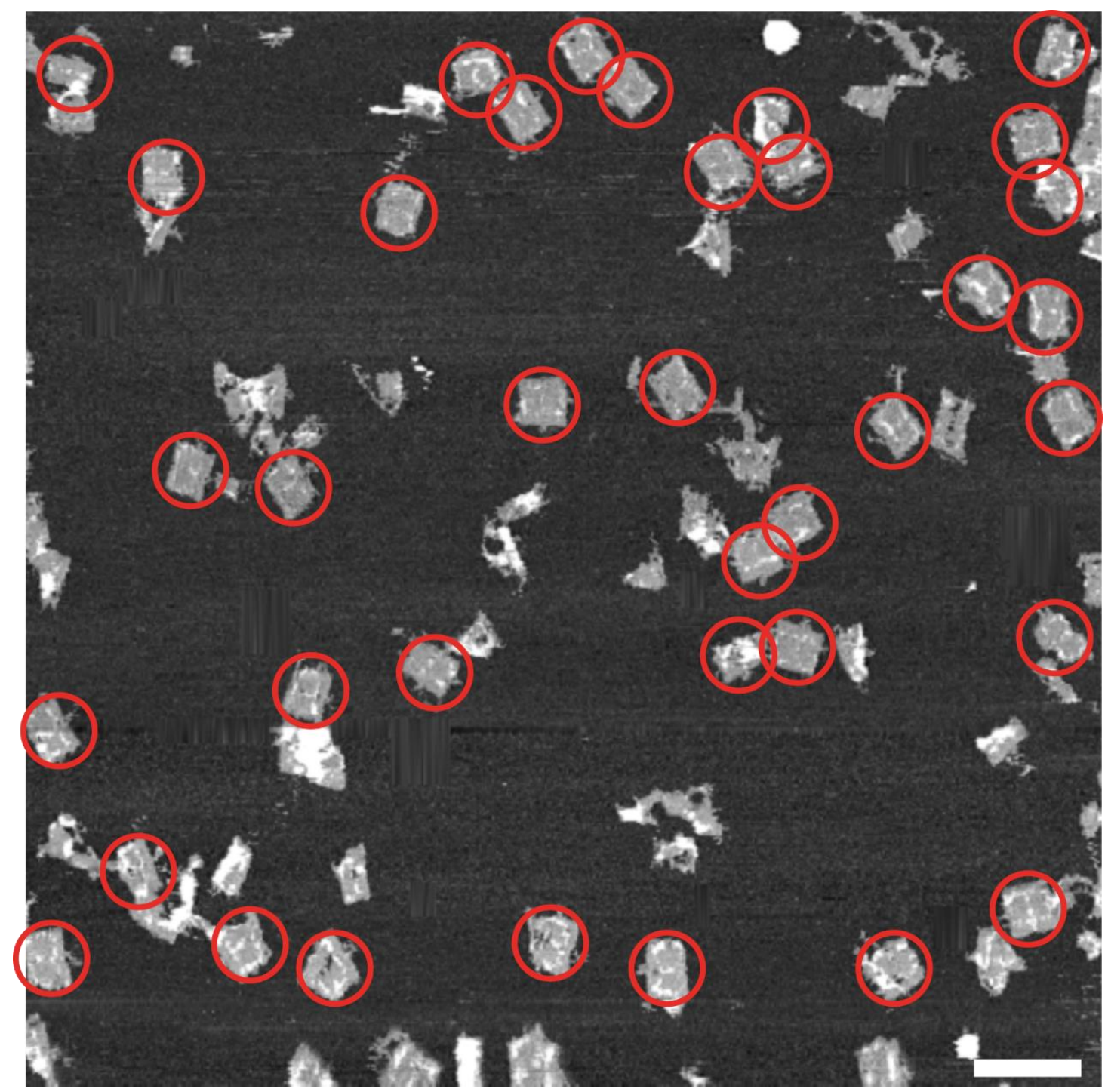

Figure S12. Silver patterns on DNA origami after a reaction of 1 min. Silver precursors: $2 \mathrm{mM}$. Reductant: glucose. Scale bar: $200 \mathrm{~nm}$. DNA origamis included in the statistics are circled in red. 


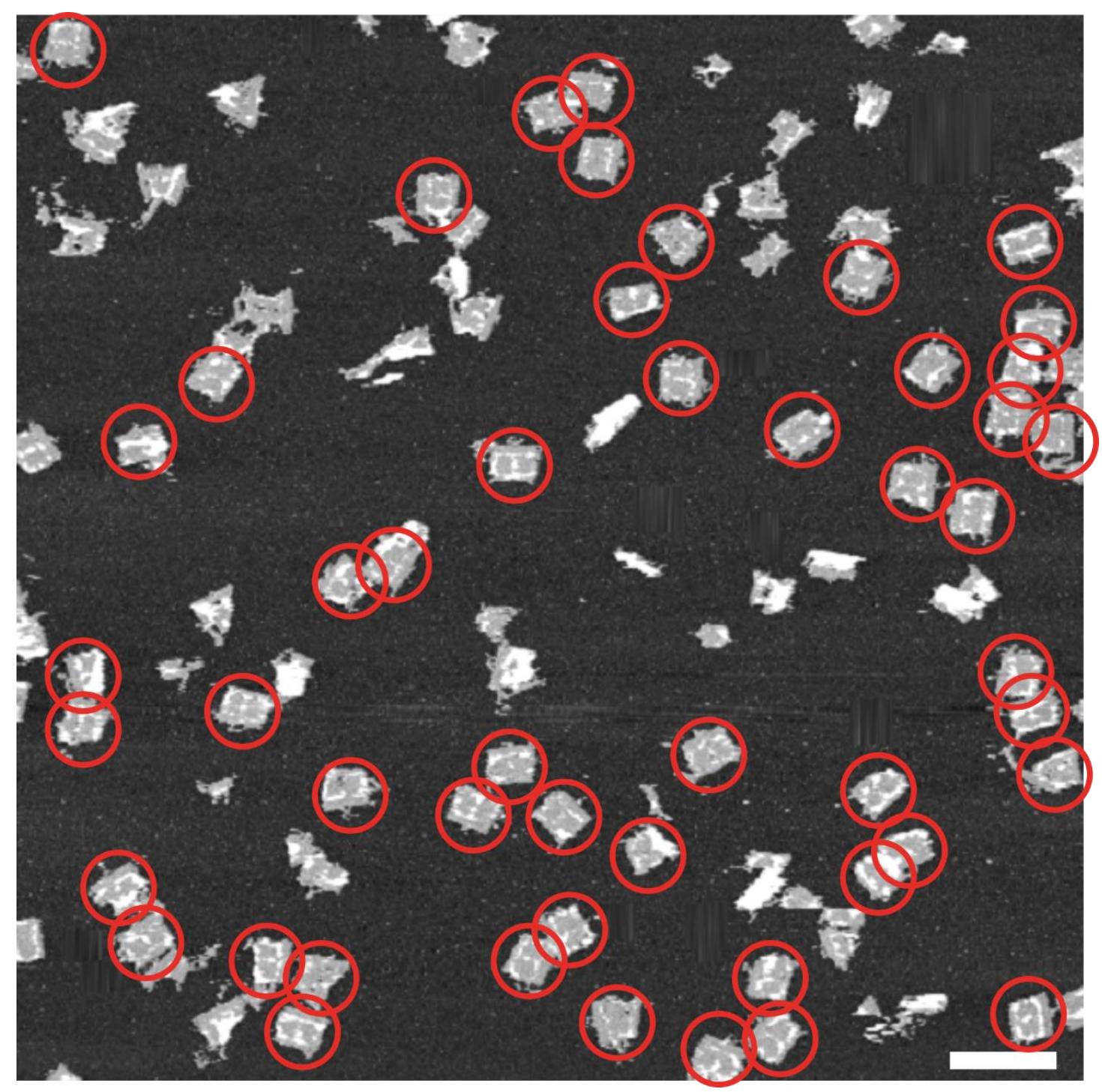

Figure S13. Silver patterns on DNA origami after a reaction of $5 \mathrm{~min}$. Silver precursors: $2 \mathrm{mM}$. Reductant: glucose. Scale bar: $200 \mathrm{~nm}$. DNA origamis included in the statistics are circled in red. 


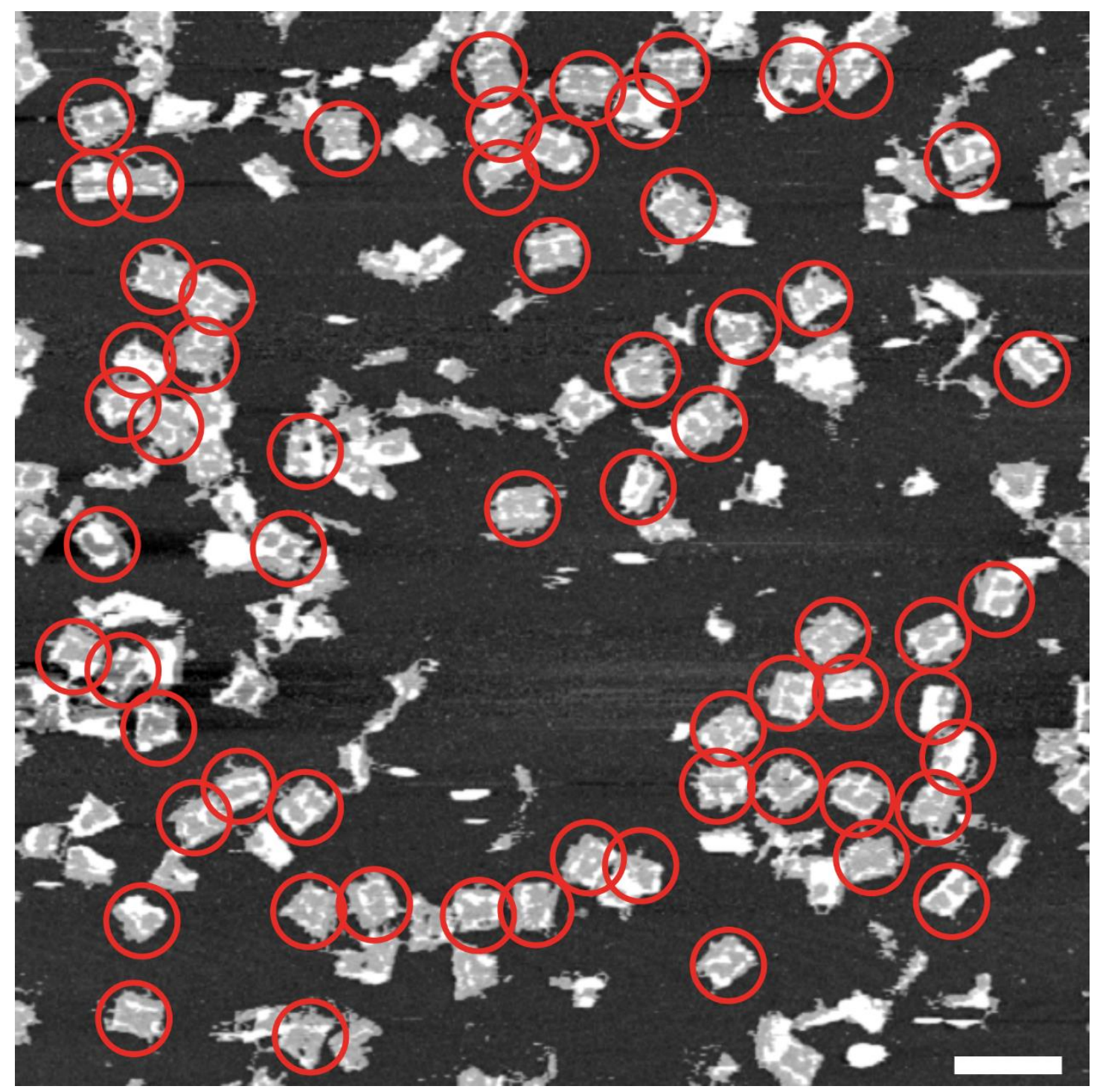

Figure S14. Silver patterns on DNA origami after a reaction of $30 \mathrm{~min}$. Silver precursors: $2 \mathrm{mM}$. Reductant: glucose. Scale bar: $200 \mathrm{~nm}$. DNA origamis included in the statistics are circled in red. 


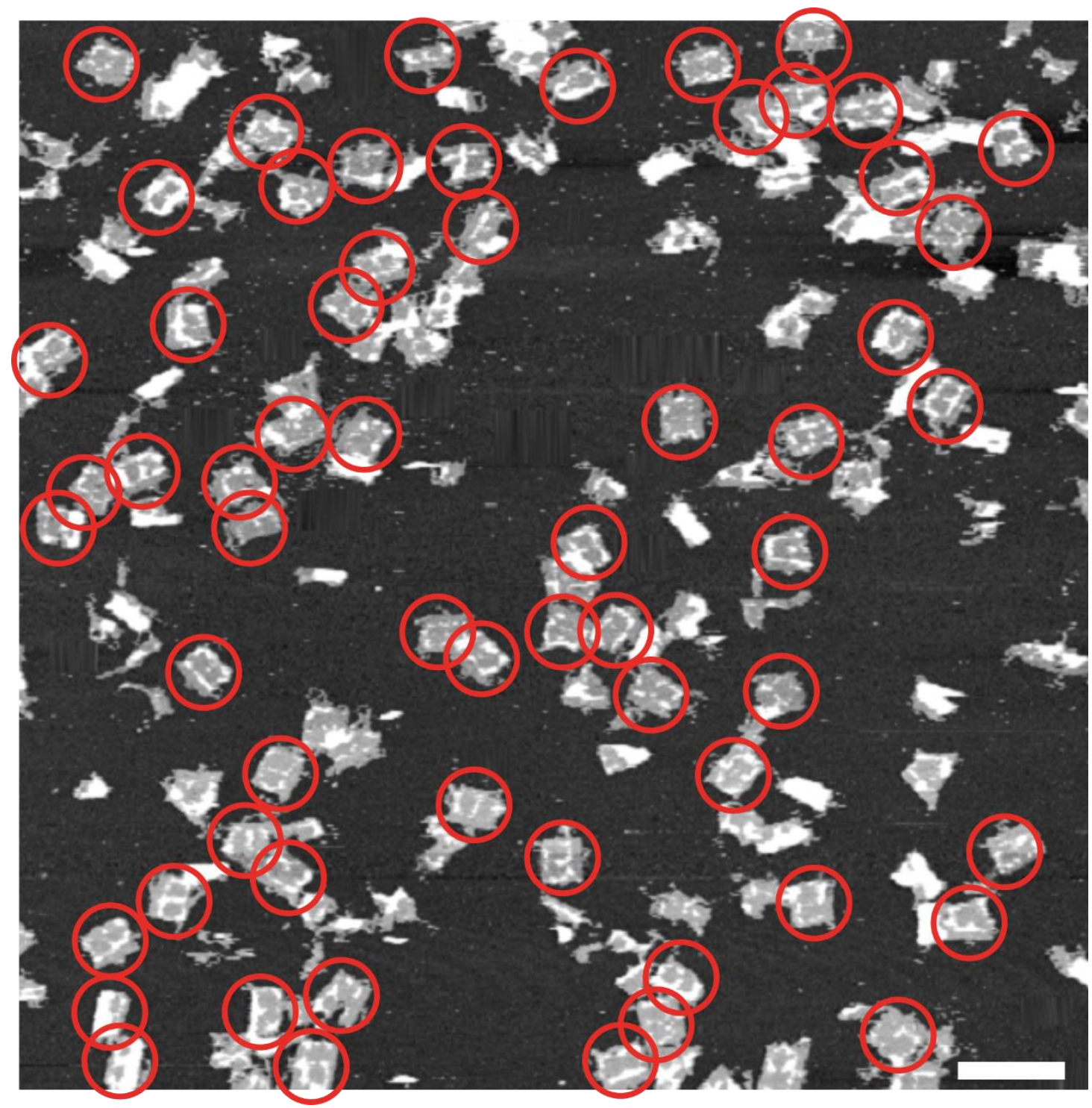

Figure S15. Silver patterns on DNA origami after a reaction of $60 \mathrm{~min}$. Silver precursors: $2 \mathrm{mM}$. Reductant: glucose. Scale bar: $200 \mathrm{~nm}$. DNA origamis included in the statistics are circled in red. 


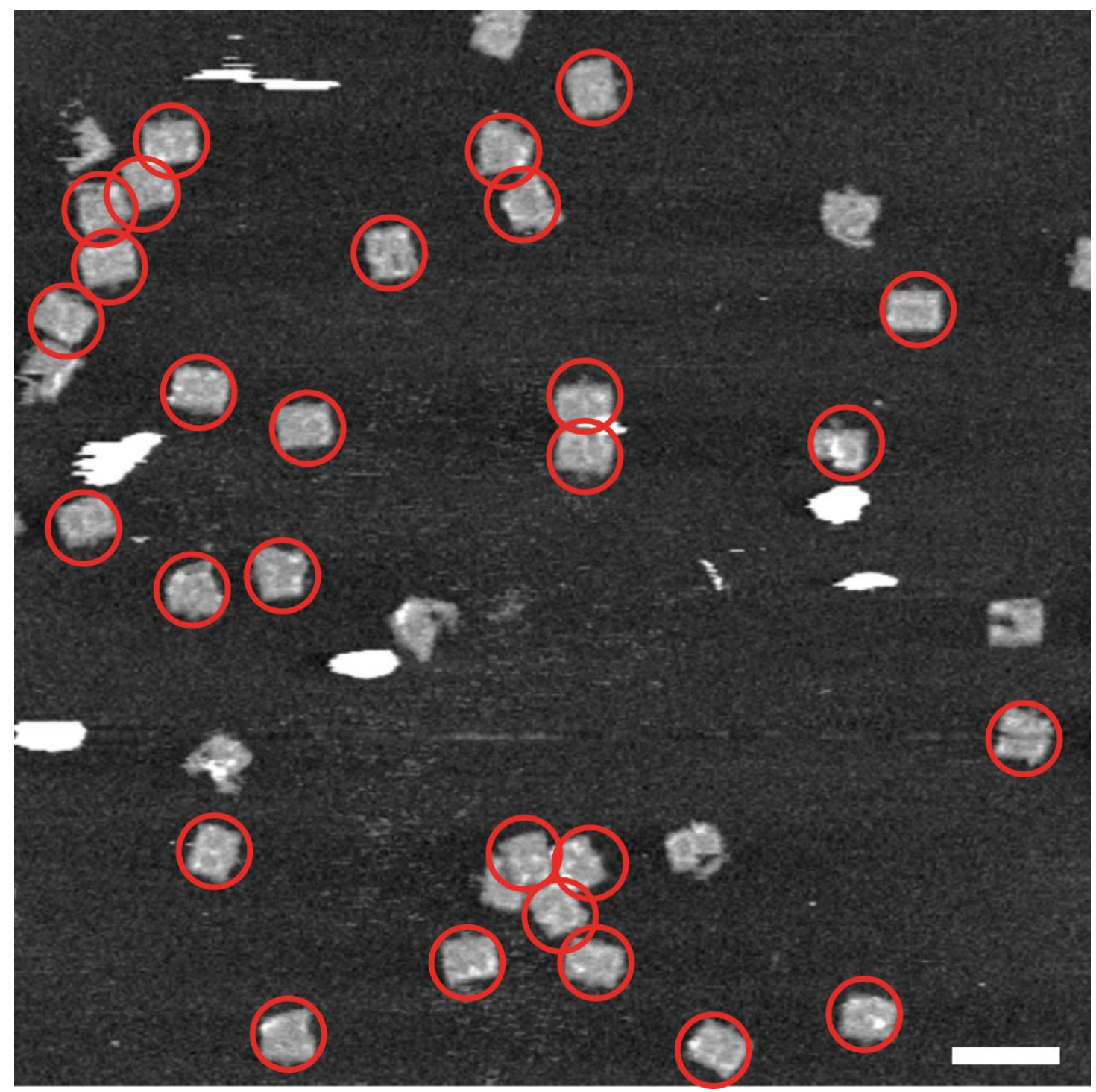

Figure S16. Silver patterns on DNA origami reduced by sodium borohydride (SB). Silver precursors: $2 \mathrm{mM}$. Reaction time: $10 \mathrm{~min}$. Scale bar: $200 \mathrm{~nm}$. DNA origamis included in the statistics are circled in red. 


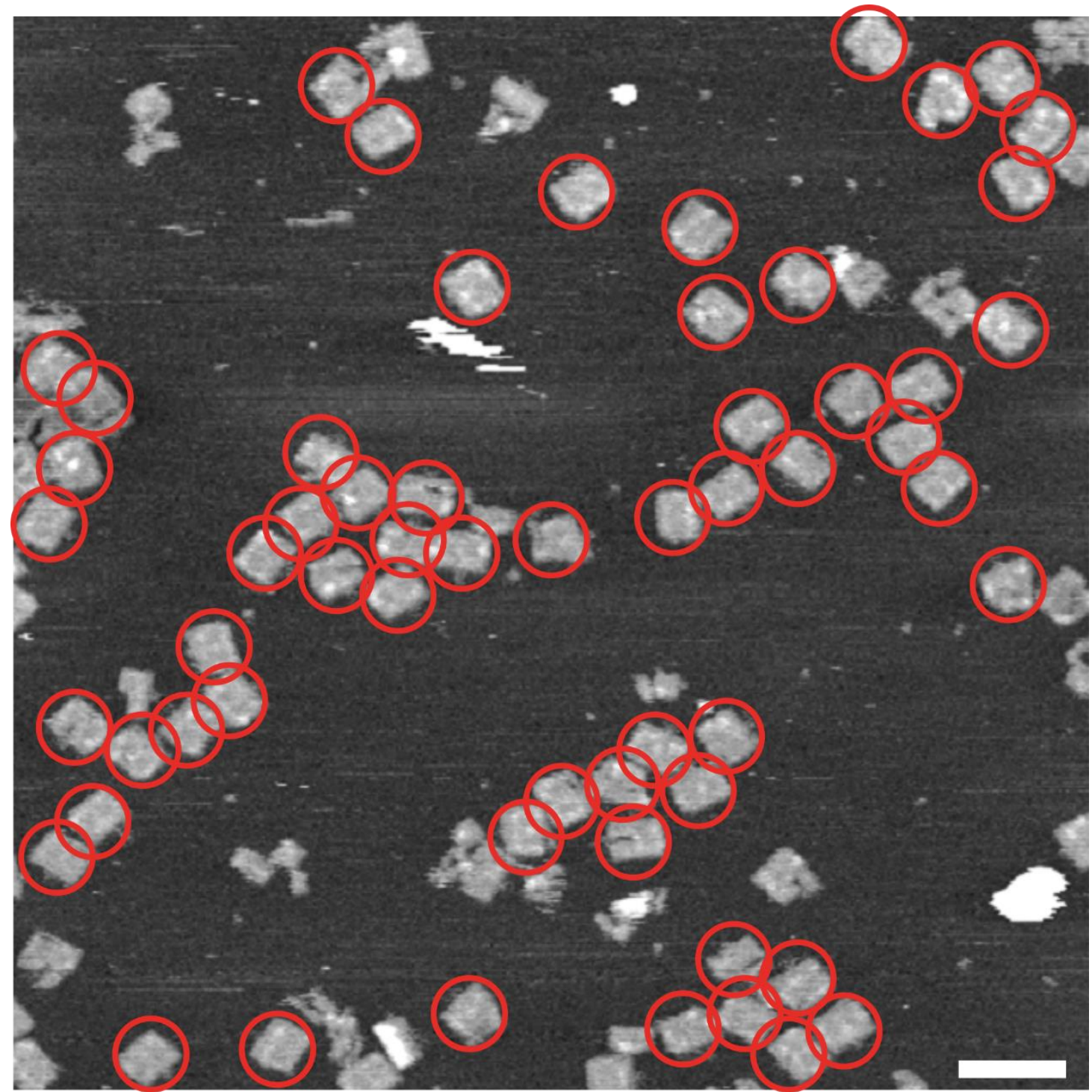

Figure S17. Silver patterns on DNA origami reduced by ascorbic acid (AA). Silver precursors: $2 \mathrm{mM}$. Reaction time: $10 \mathrm{~min}$. Scale bar: $200 \mathrm{~nm}$. DNA origamis included in the statistics are circled in red. 


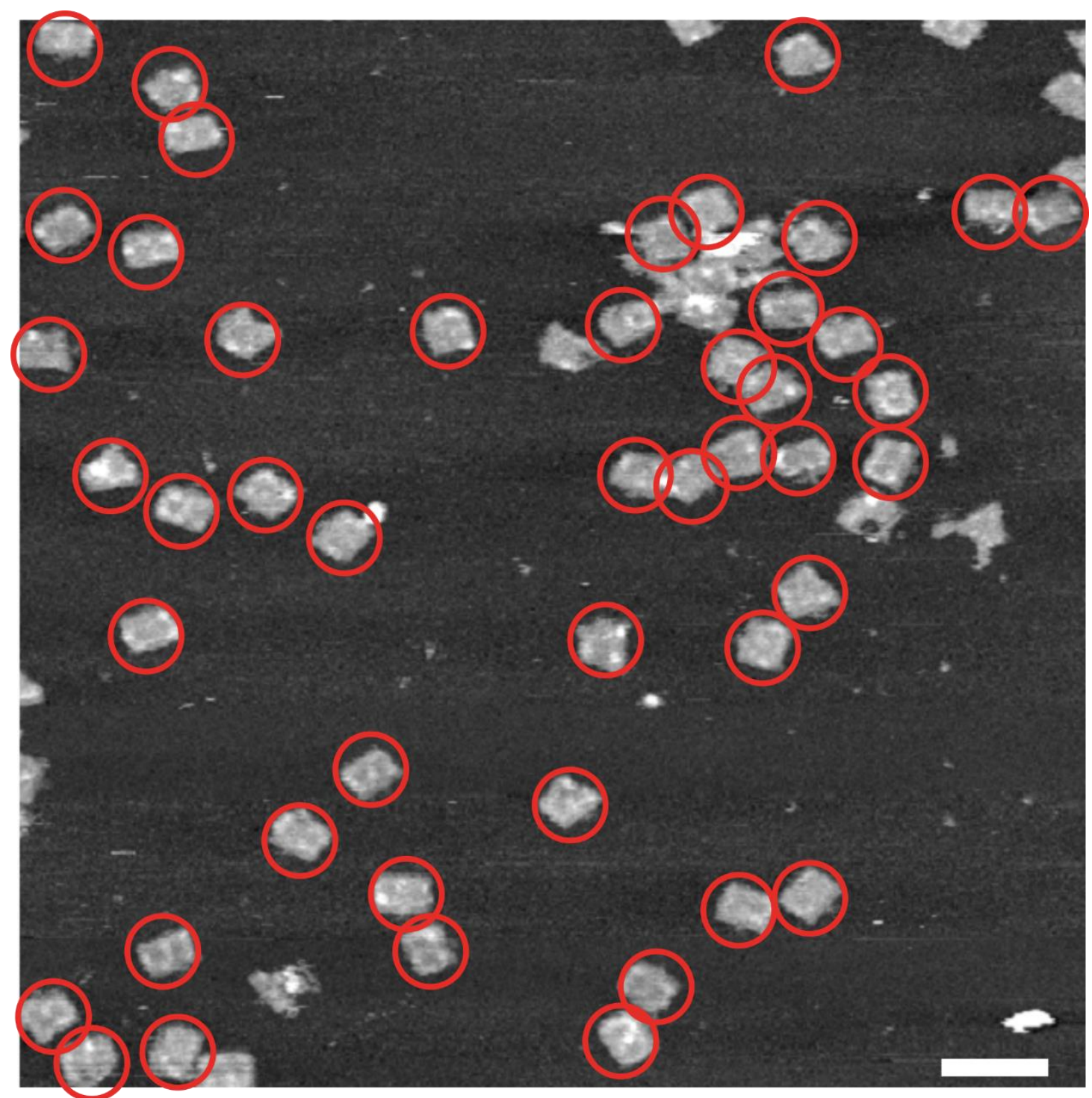

Figure S18. Silver patterns on DNA origami reduced by hydroxylamine (HA). Silver precursors: $2 \mathrm{mM}$. Reaction time: $10 \mathrm{~min}$. Scale bar: $200 \mathrm{~nm}$. DNA origamis included in the statistics are circled in red. 


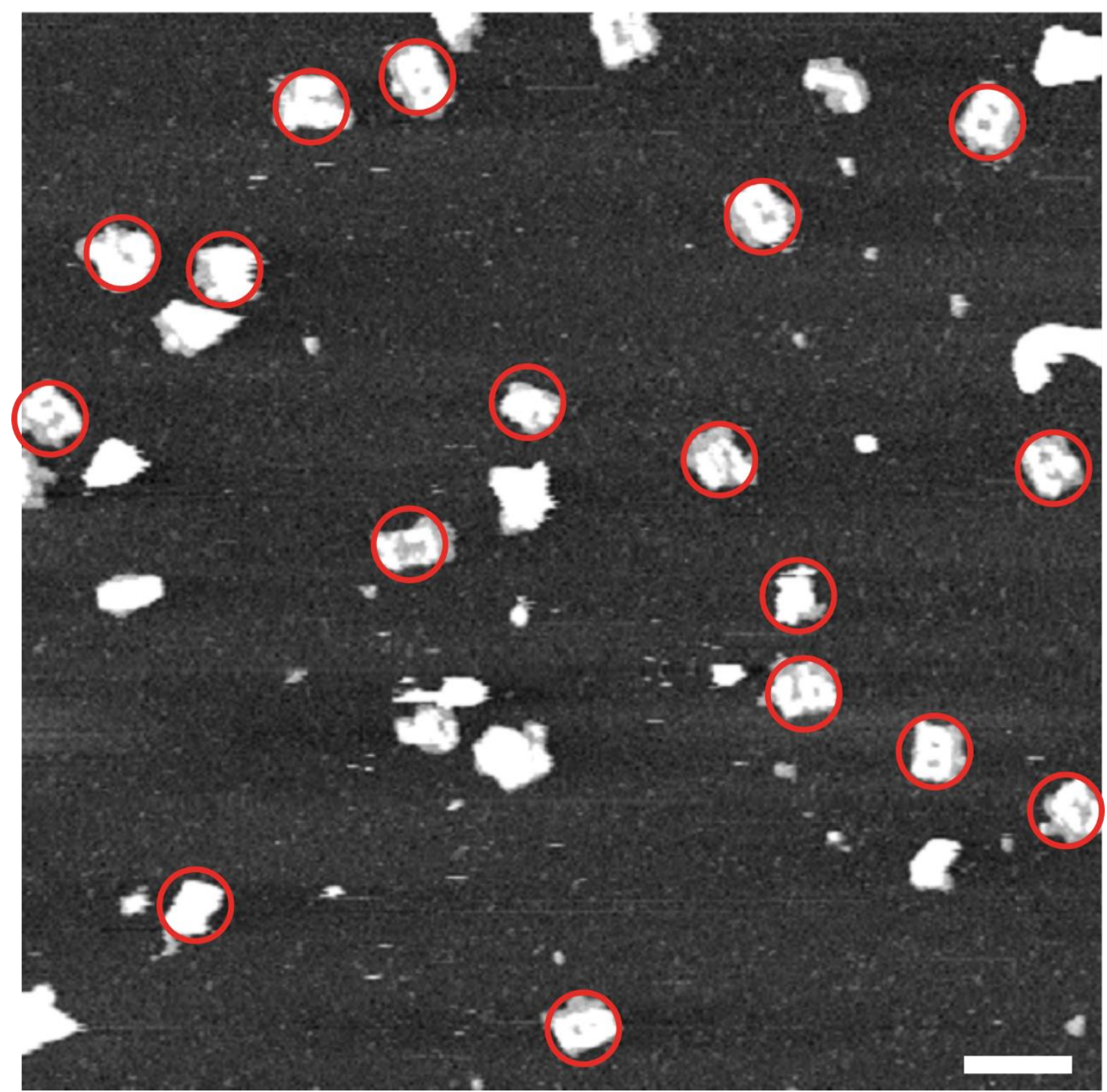

Figure S19. Silver patterns on DNA origami reduced by glutaraldehyde (GA). Silver precursors: $2 \mathrm{mM}$. Reaction time: $10 \mathrm{~min}$. Scale bar: $200 \mathrm{~nm}$. DNA origamis included in the statistics are circled in red. 


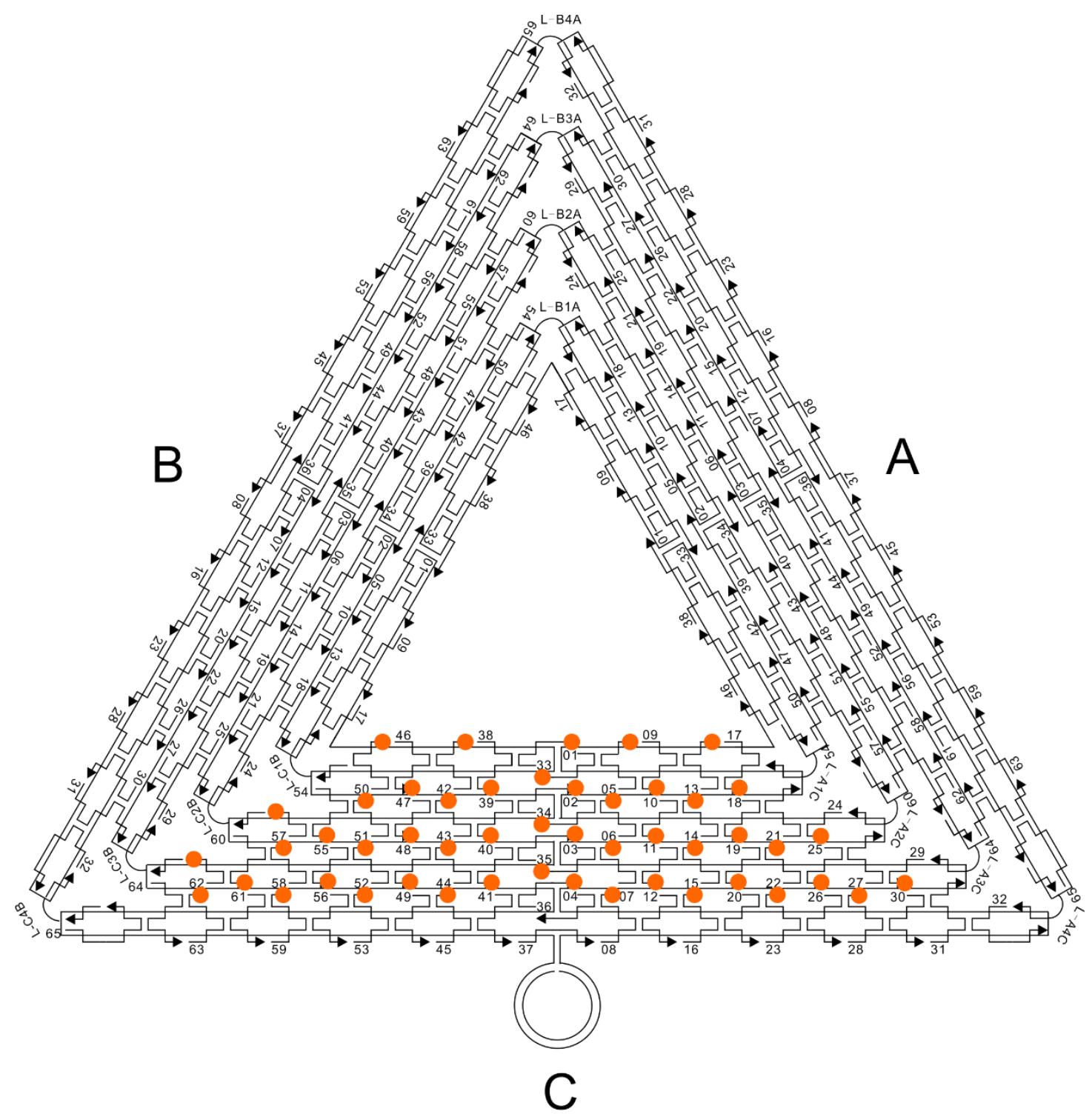

Figure S20. Schematic design of pcDNA blueprint on one edge of triangular DNA origami. DNA protrusions are denoted by orange dots. 


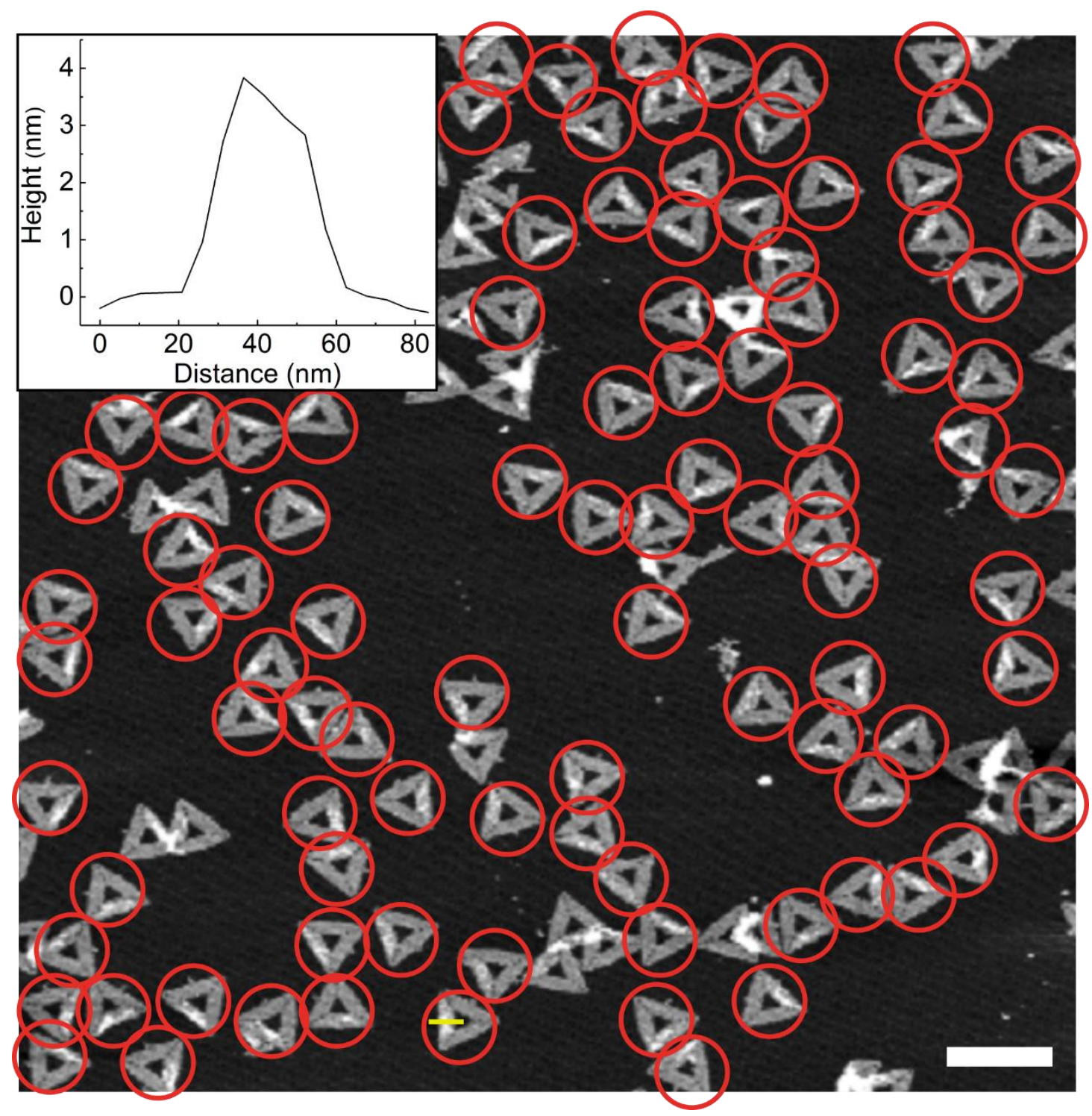

Figure S21. Silver patterns on DNA origami with 10-nt protrusion on one edge of the triangle. Silver precursors: $2 \mathrm{mM}$. Reaction time: $10 \mathrm{~min}$. Reductant: glucose. Scale bar: $200 \mathrm{~nm}$. DNA origamis included in the statistics are circled in red. Inset denotes the height plot along the yellow line. 


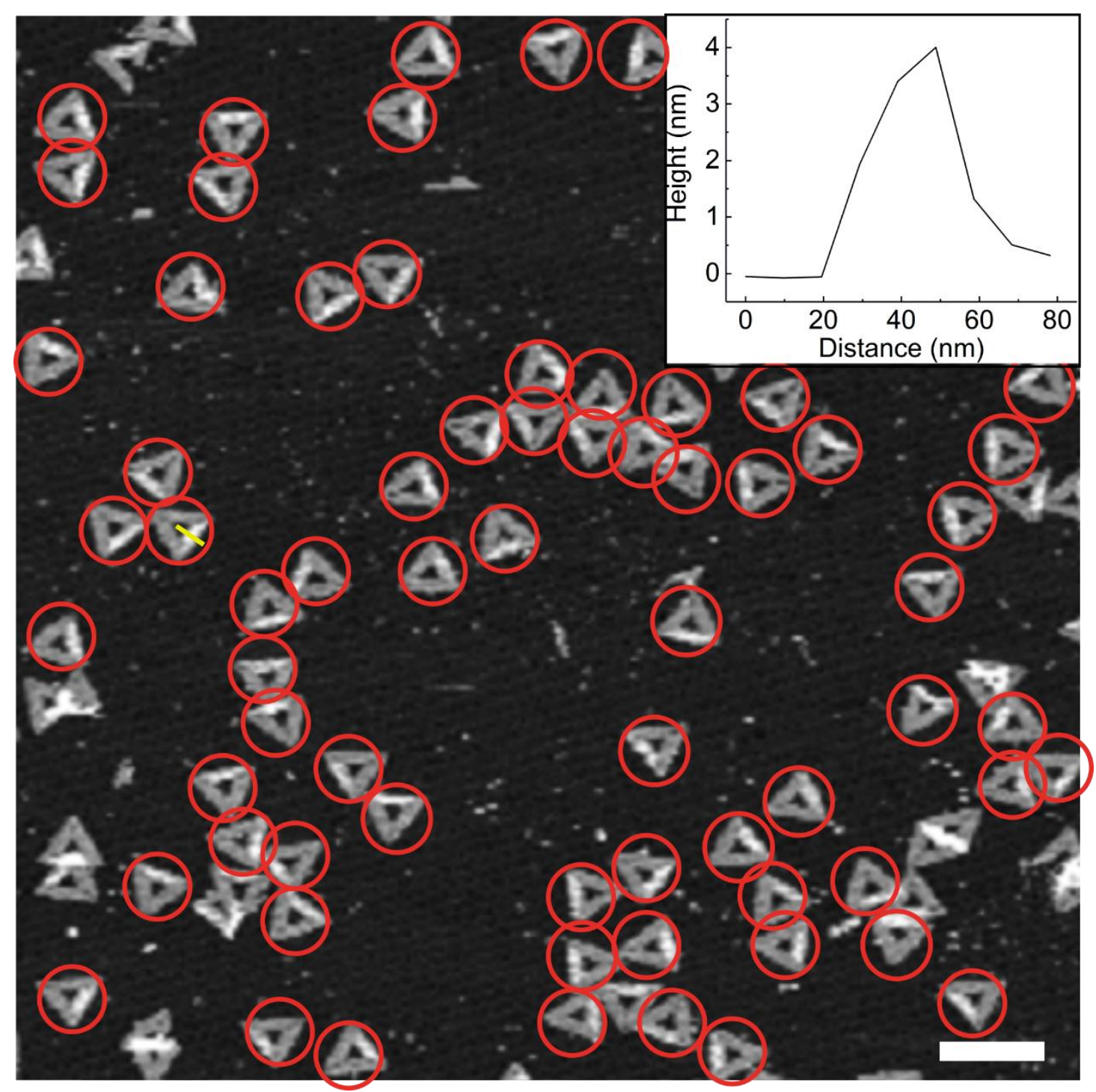

Figure S22. Silver patterns on DNA origami with 15-nt protrusion on one edge of the triangle. Silver precursors: $2 \mathrm{mM}$. Reaction time: $10 \mathrm{~min}$. Reductant: glucose. Scale bar: $200 \mathrm{~nm}$. DNA origamis included in the statistics are circled in red. Inset denotes the height plot along the yellow line. 


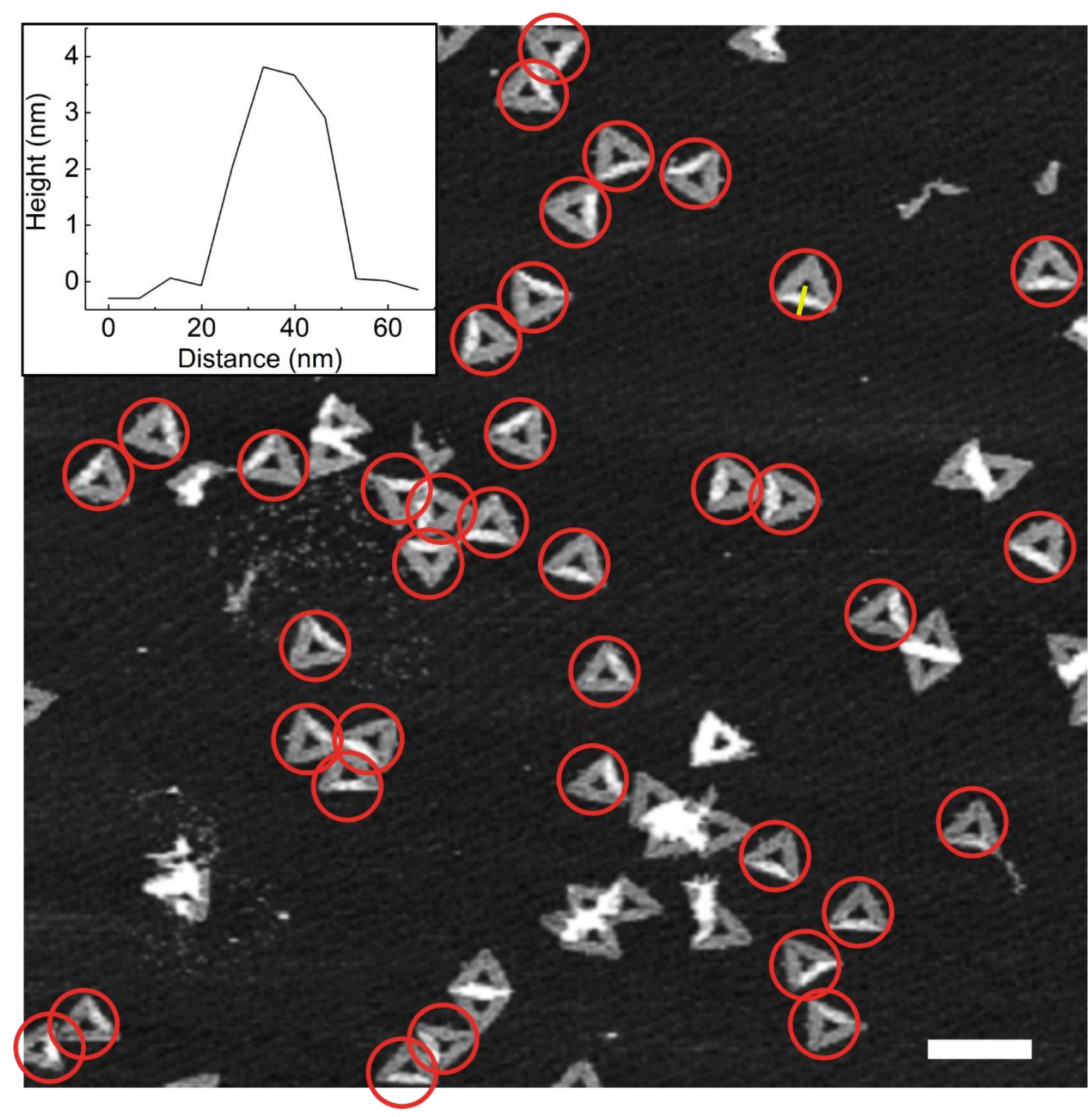

Figure S23. Silver patterns on DNA origami with 20-nt protrusion on one edge of the triangle. Silver precursors: $2 \mathrm{mM}$. Reaction time: $10 \mathrm{~min}$. Reductant: glucose. Scale bar: $200 \mathrm{~nm}$. DNA origamis included in the statistics are circled in red. Inset denotes the height plot along the yellow line. 


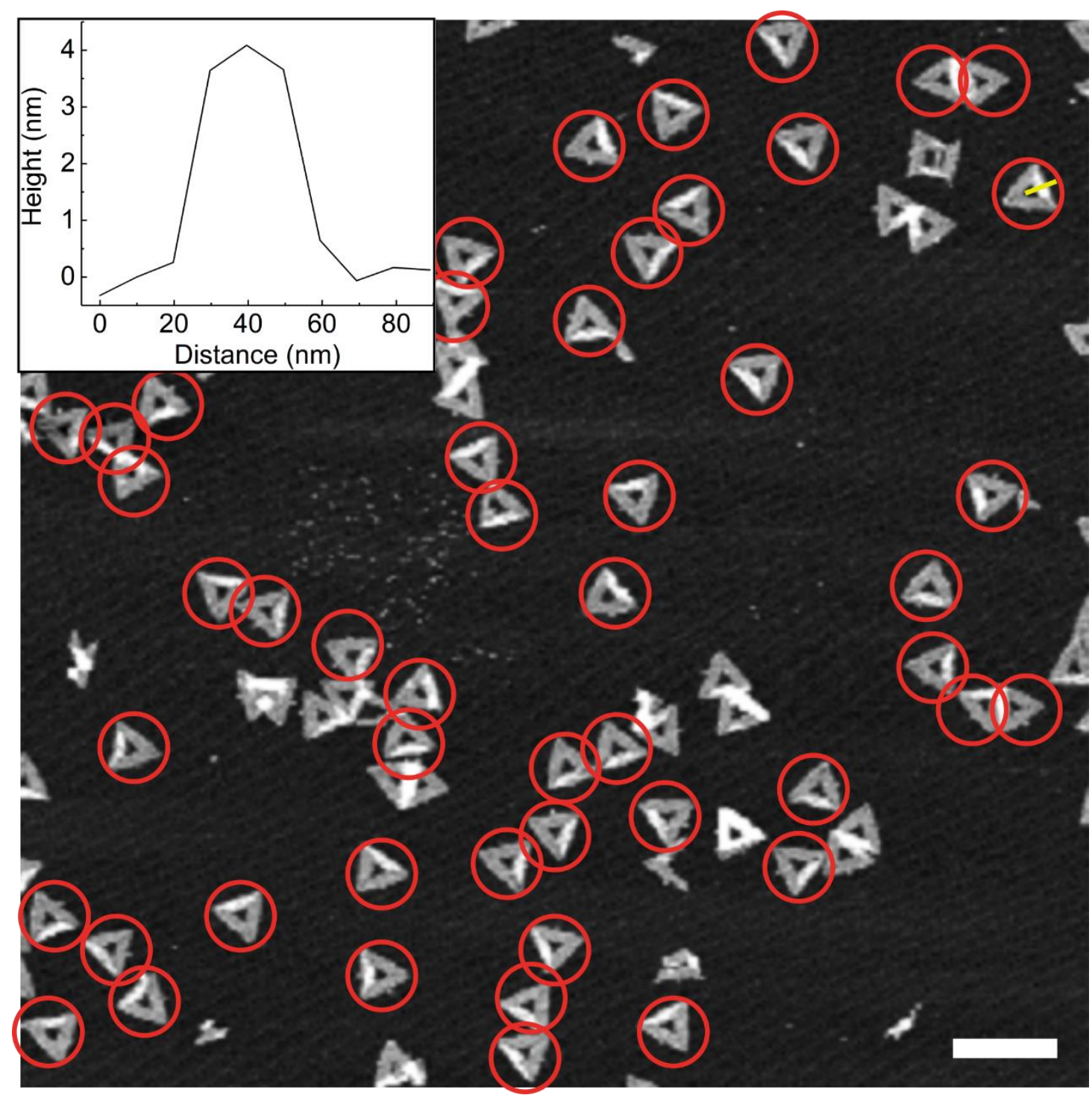

Figure S24. Silver patterns on DNA origami with 30-nt protrusion on one edge of the triangle. Silver precursors: $2 \mathrm{mM}$. Reaction time: $10 \mathrm{~min}$. Reductant: glucose. Scale bar: $200 \mathrm{~nm}$. DNA origamis included in the statistics are circled in red. Inset denotes the height plot along the yellow line. 


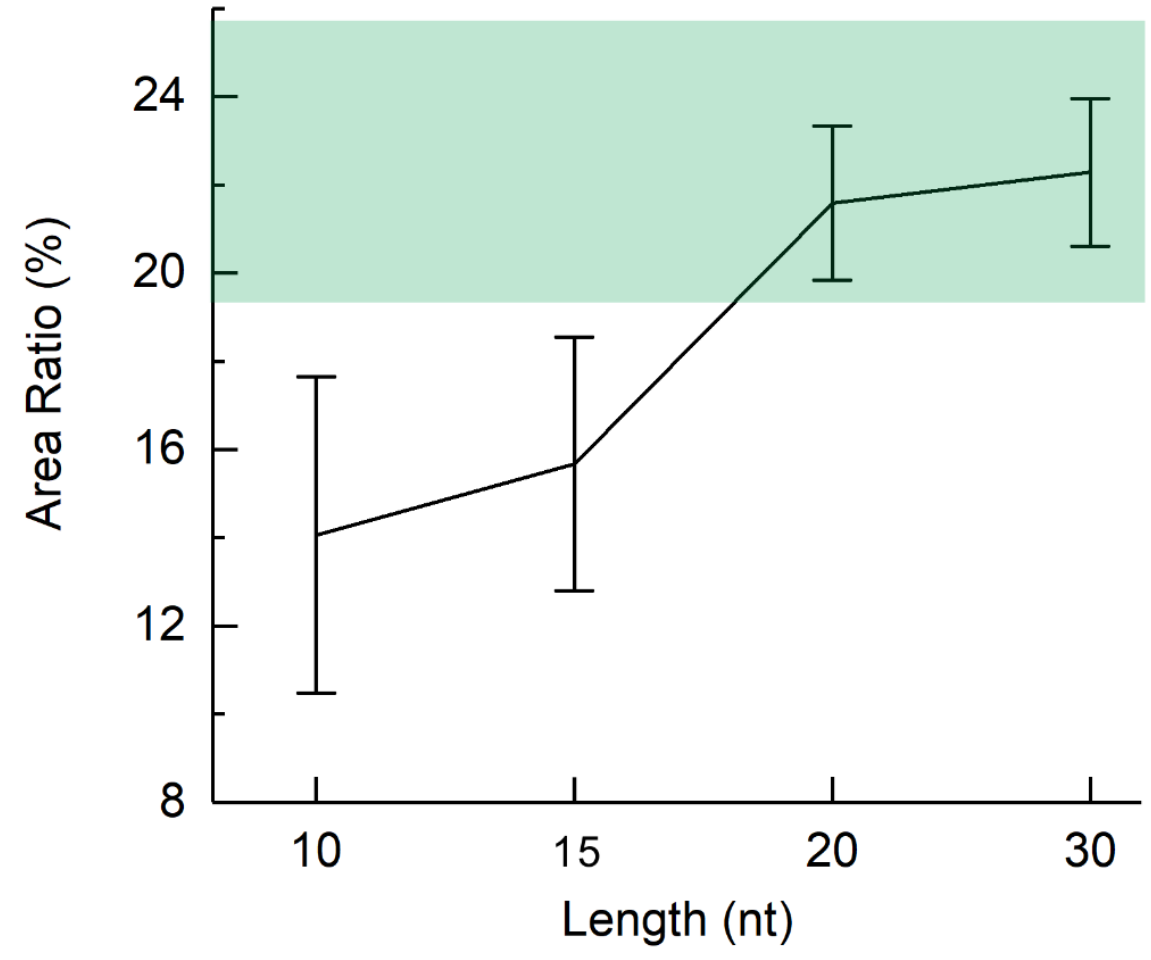

Figure S25. Area ratios of silver coverage to that of the osDNA when protrusions of different lengths were used. The green box denotes the expected range estimated from the pcDNA blueprint. Error bars indicate the standard deviation of three independent measurements. 


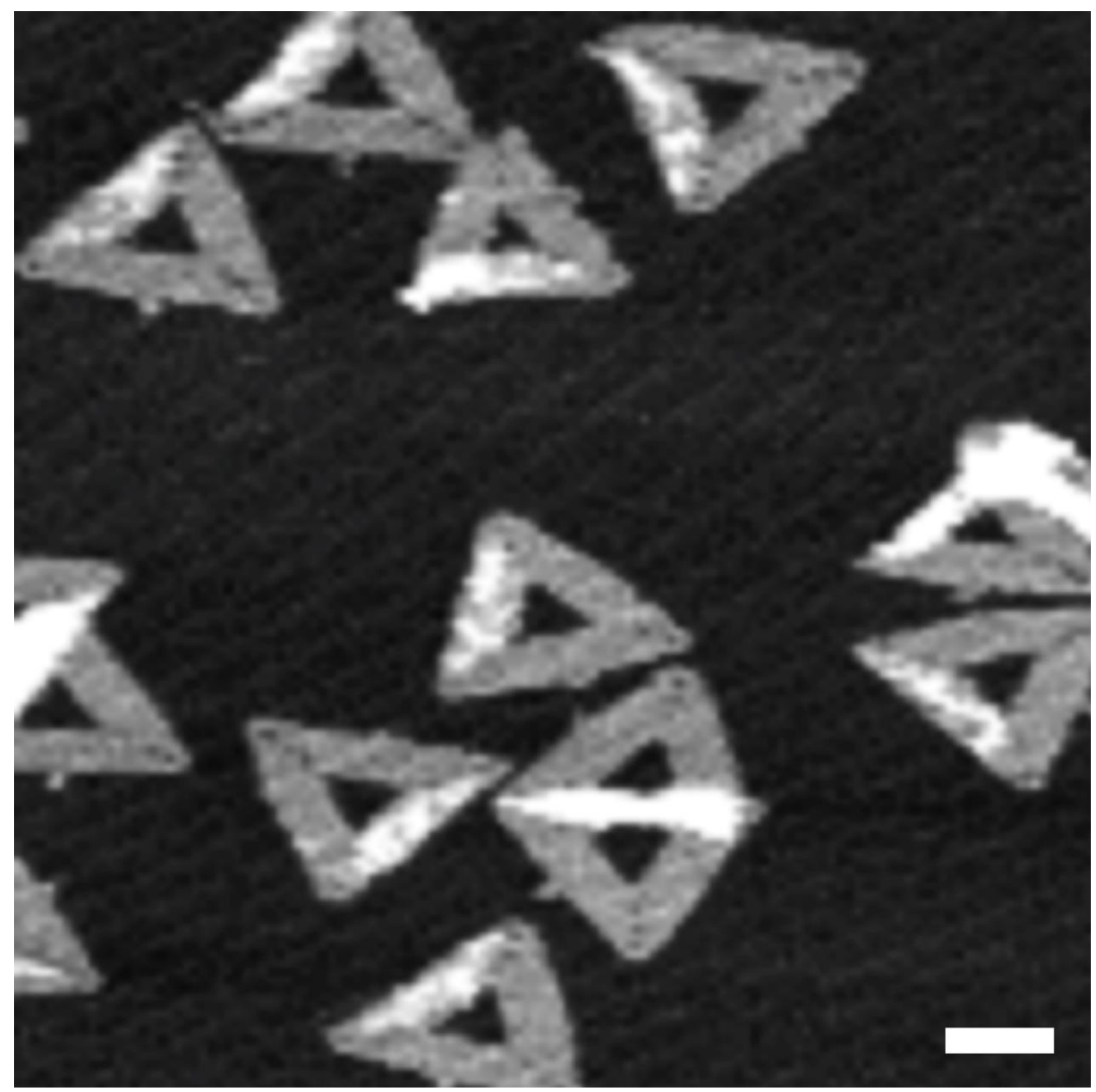

Figure S26. Silver patterns on one edge of DNA origami at a higher magnification. Protrusion: $20 \mathrm{nt}$. Silver precursors: $2 \mathrm{mM}$. Reaction time: $10 \mathrm{~min}$. Reductant: glucose. Scale bar: $50 \mathrm{~nm}$. 


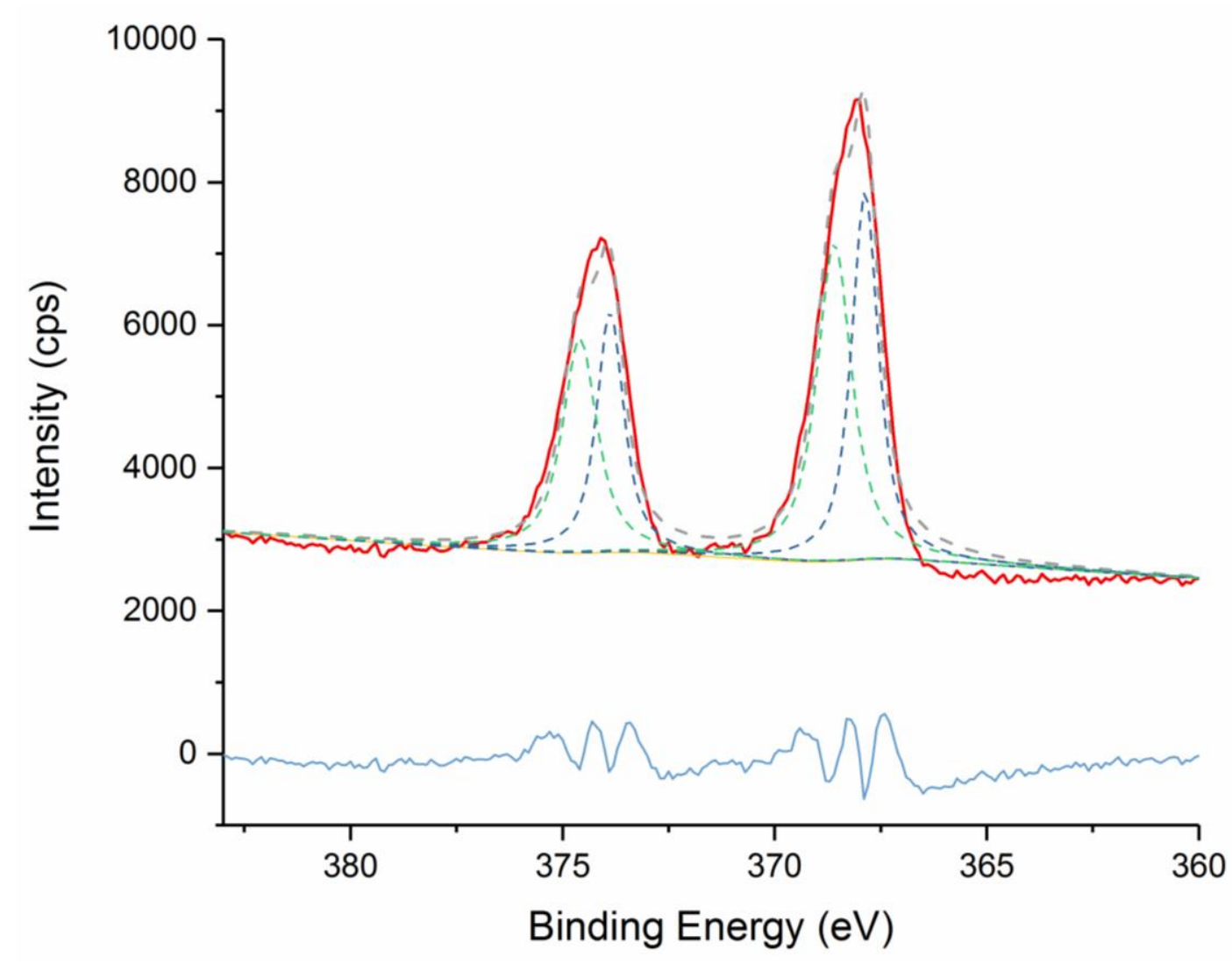

Figure S27. X-ray photoelectron spectra of silver patterns on DNA origami. The dashed lines denoted in green and blue indicate the signals from $\operatorname{Ag}(0)$ and $\operatorname{Ag}(\mathrm{I})$ species, respectively. 


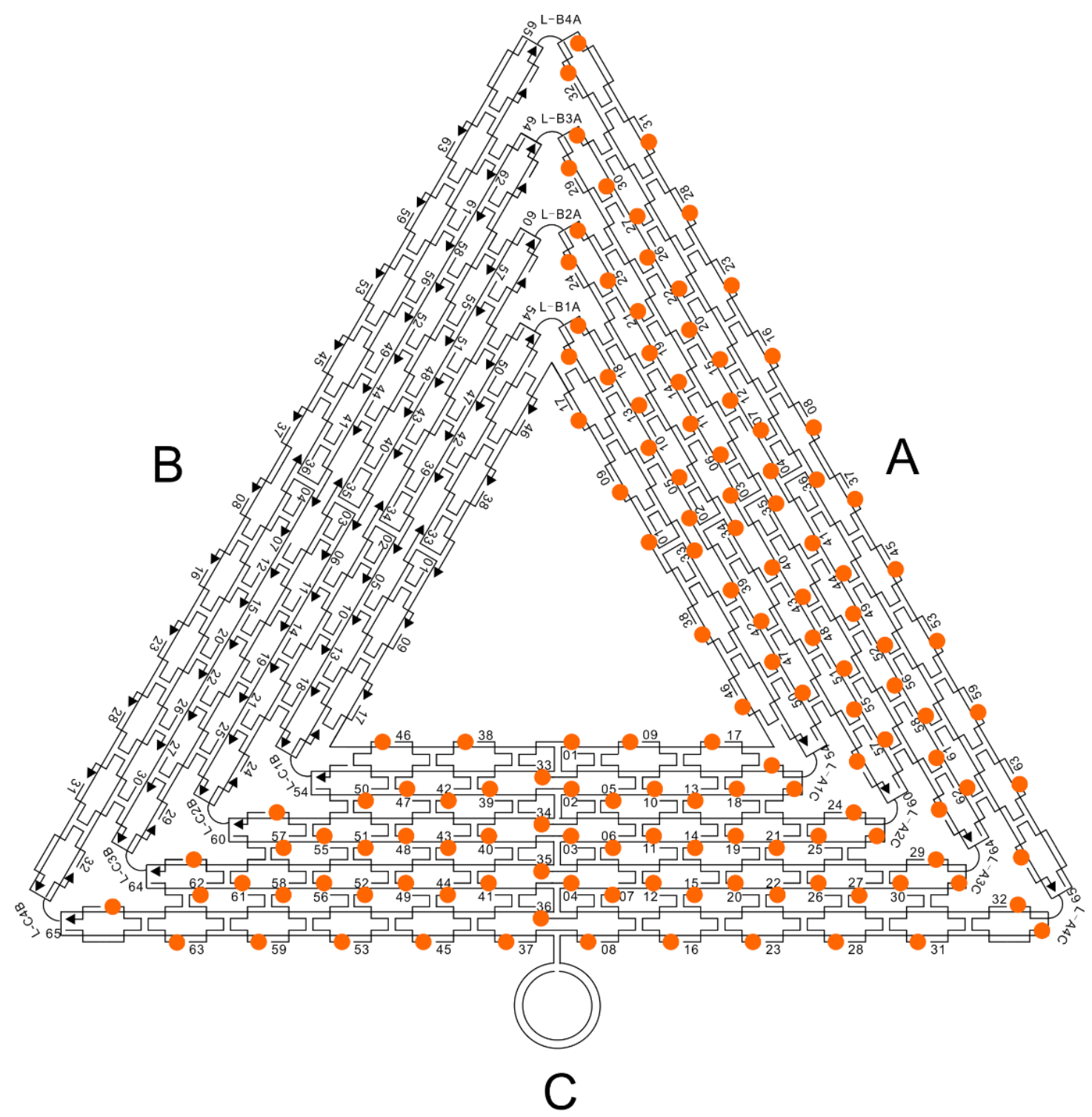

Figure S28. Schematic design of bilateral pcDNA template on triangular DNA origami. DNA protrusions are denoted by orange dots. 

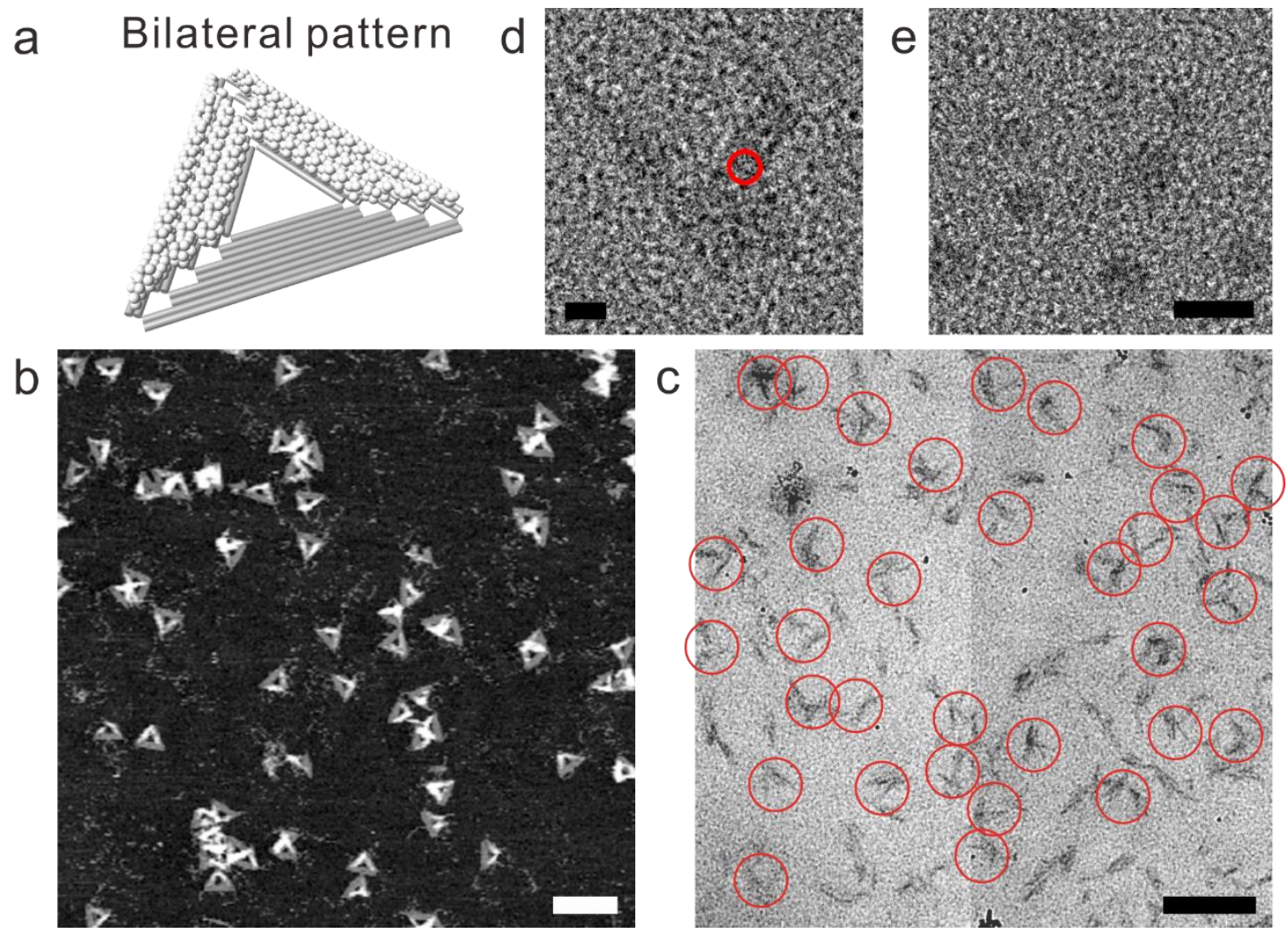

Figure S29. AFM and TEM images of bilateral silver patterns on triangular DNA origami. (a) Schematic cartoon of the silver pattern on osDNA. (b) AFM image of the silver pattern at a large view. (c) TEM image of silver patterns at a large view. (d) Magnified view of a single bilateral silver pattern. (e) High-resolution image of the selected area denoted in red in (d). Scale bar: (b) and (c) $200 \mathrm{~nm}$, (d) $20 \mathrm{~nm}$, (e) $5 \mathrm{~nm}$. 

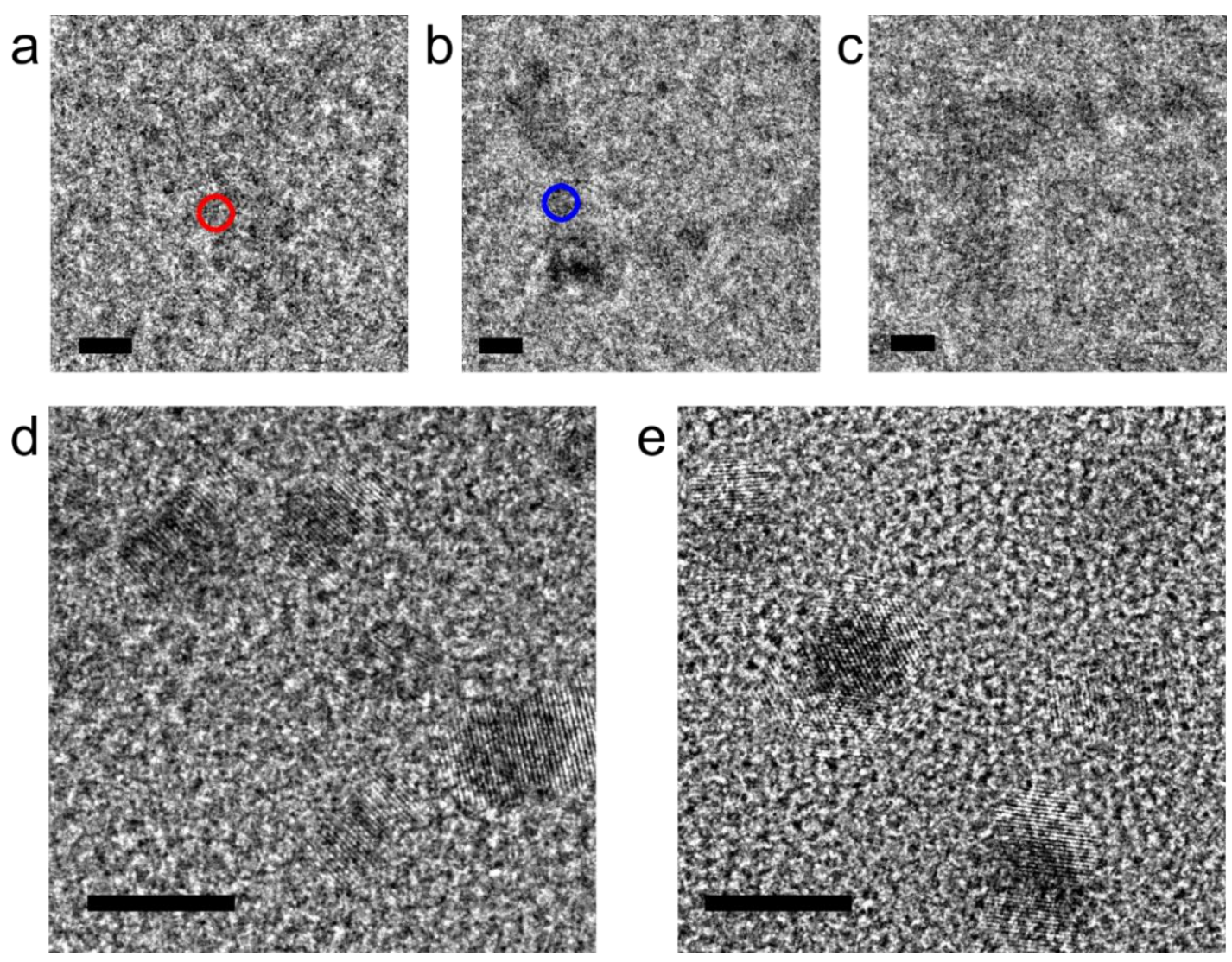

Figure S30. Typical TEM images with higher magnification. (a)-(c) Single bilateral silver patterns. (d) High-resolution image of the selected area denoted in red in (a). (e) High-resolution image of the selected area denoted in blue in (b). Scale bars: (a)-(c) 20 $\mathrm{nm},(\mathrm{d})$ and (e) $5 \mathrm{~nm}$. 


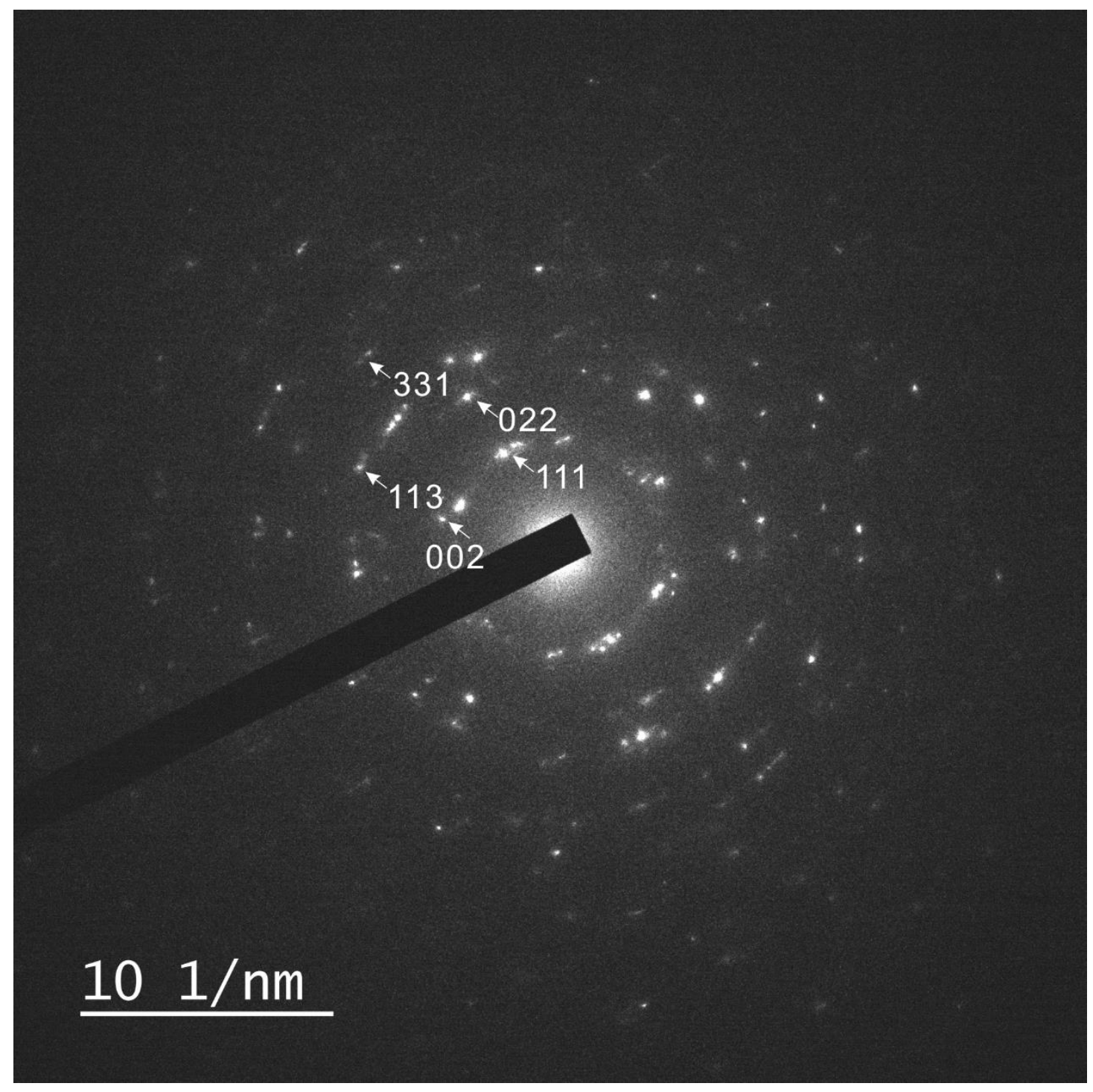

Figure S31. SAED image of silver bilateral silver patterns on triangular DNA origami. 


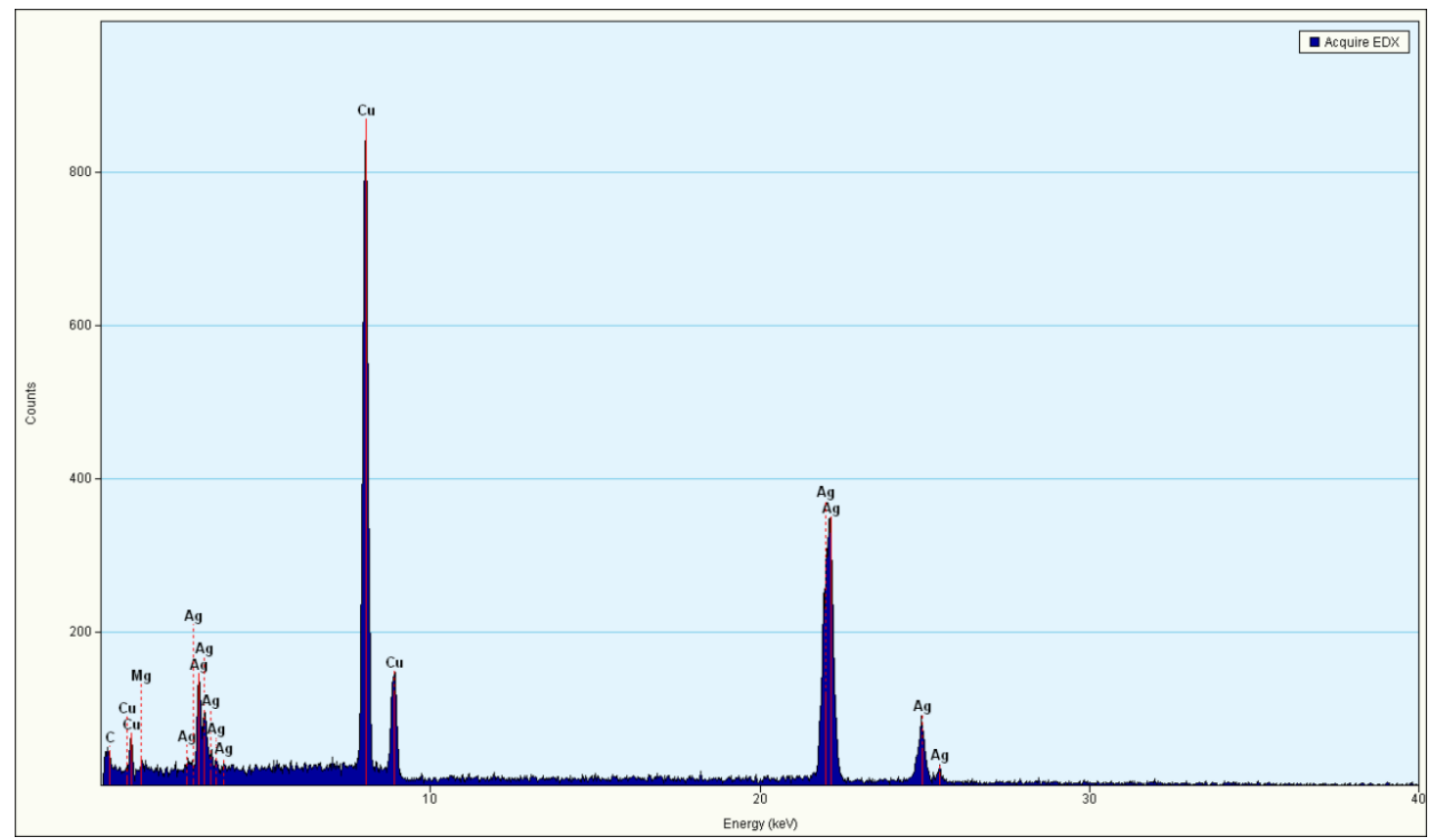

Figure S32. EDS spectrum of the bilateral silver pattern on triangular DNA origami. The signal of copper element is from the TEM grid. 


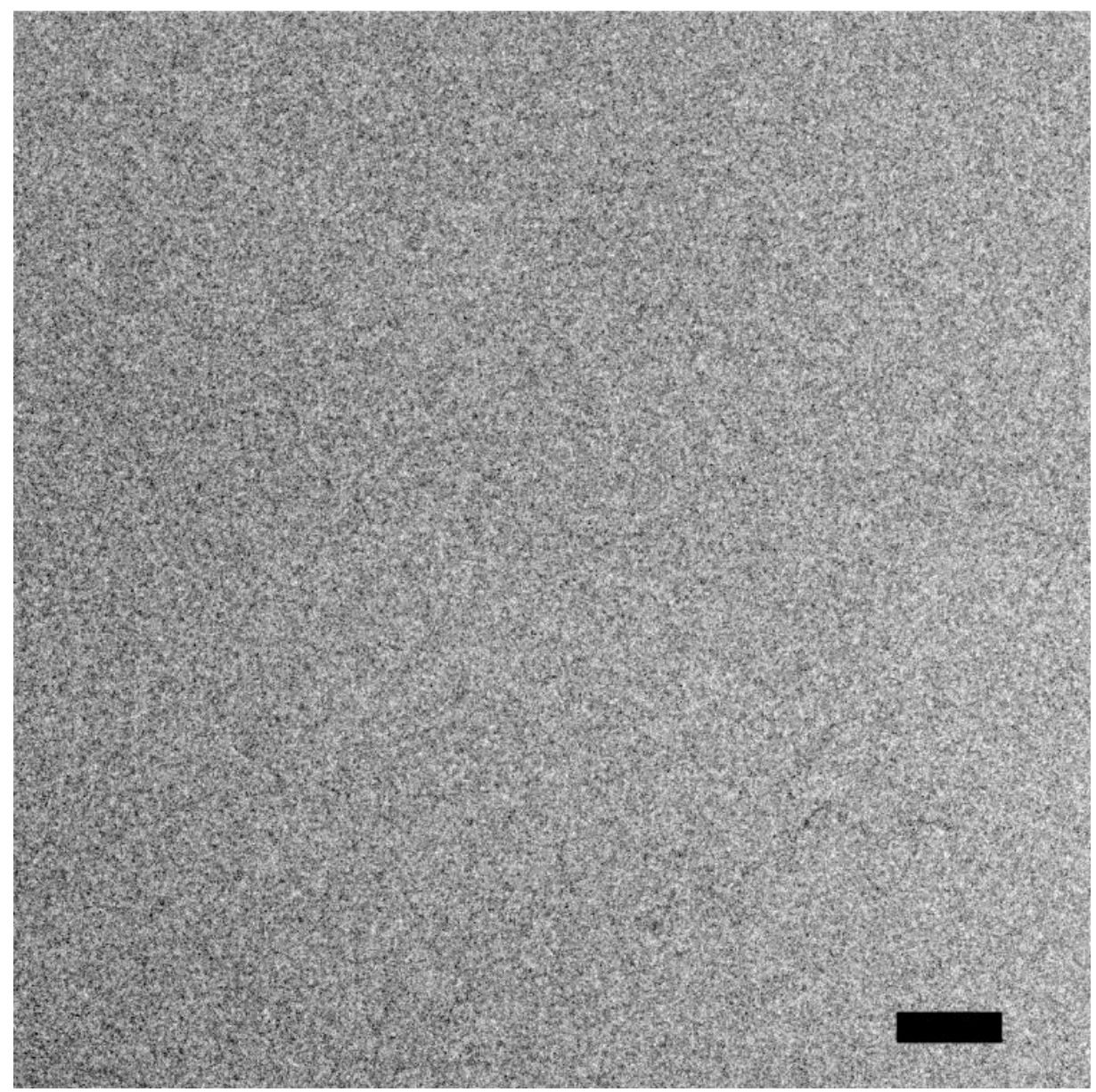

Figure S33. TEM images of unmetallized triangular DNA origami with a bilateral pcDNA blueprint. Scale bar: $200 \mathrm{~nm}$. 


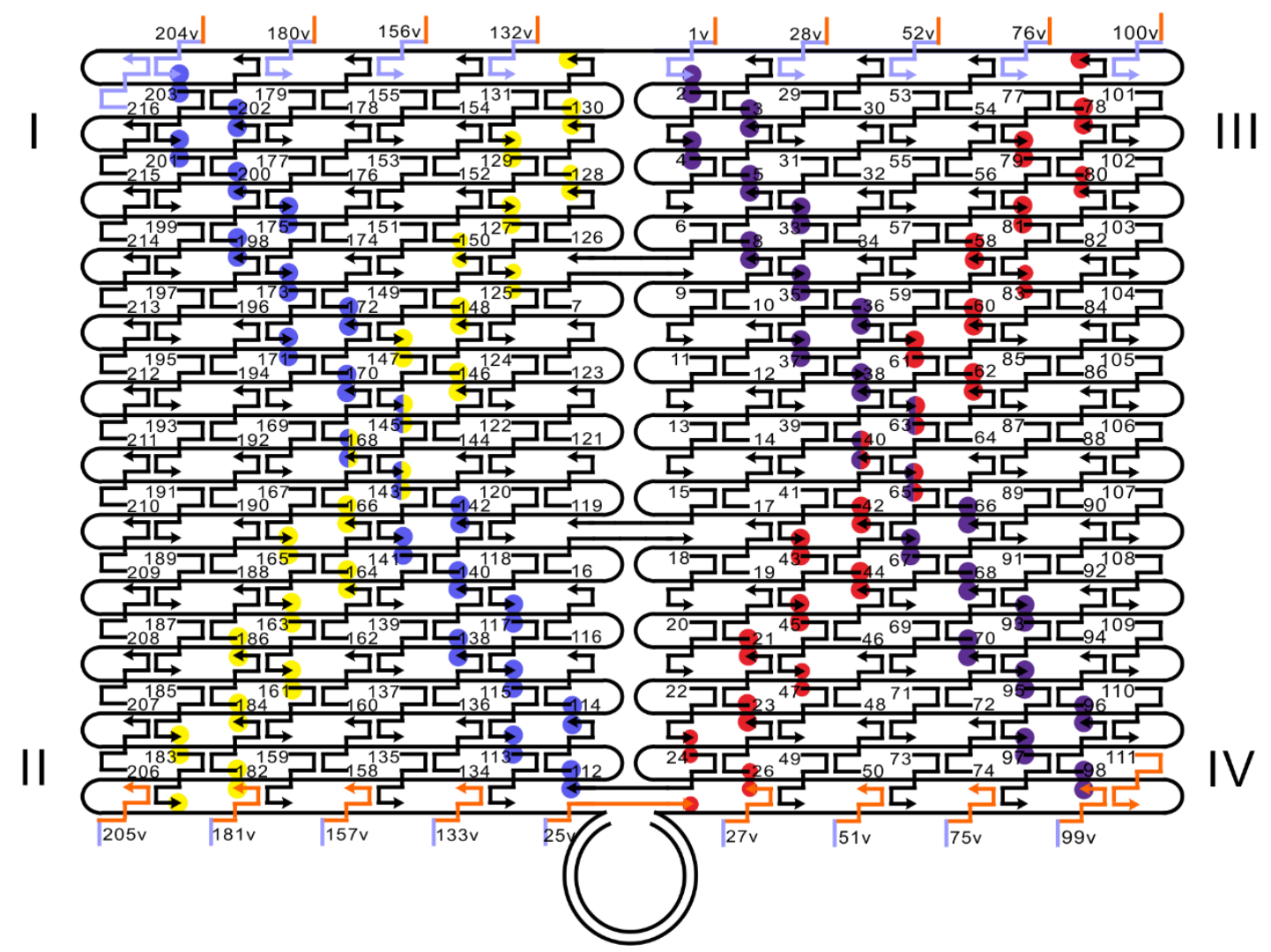

Figure S34. Schematic design of helical pcDNA templates with a pitch of $50 \mathrm{~nm}$ on DNA origami. Four lines of pcDNA strands (Form I to IV) are placed for regulation of the silver chirality. Eight bases were exchanged between the staples at the bottom and top of the DNA origami to roll the rectangle into a tube. 

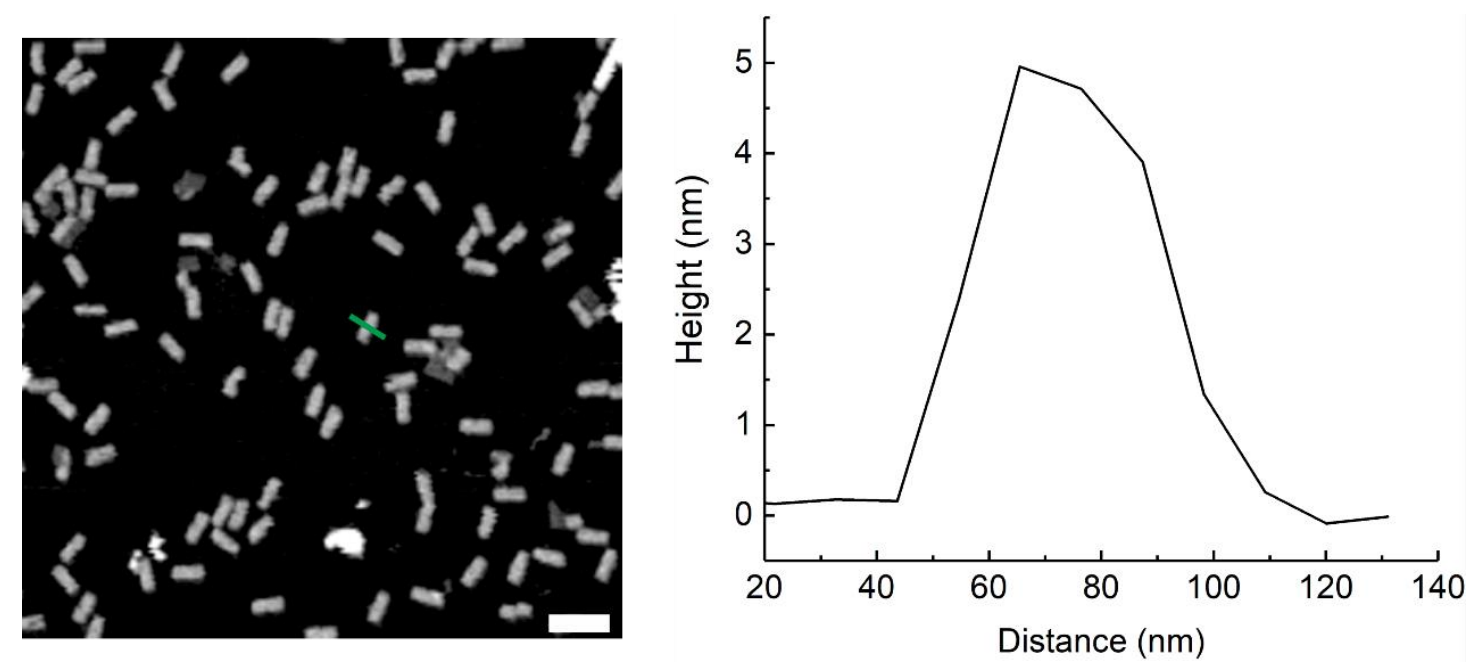

Figure S35. AFM image of tubular DNA origami. The transection height profile along the green line is given in the right panel. Under pressure by the AFM tip, the DNA origami appeared like double-layer rectangle. Scale bar: $200 \mathrm{~nm}$. 


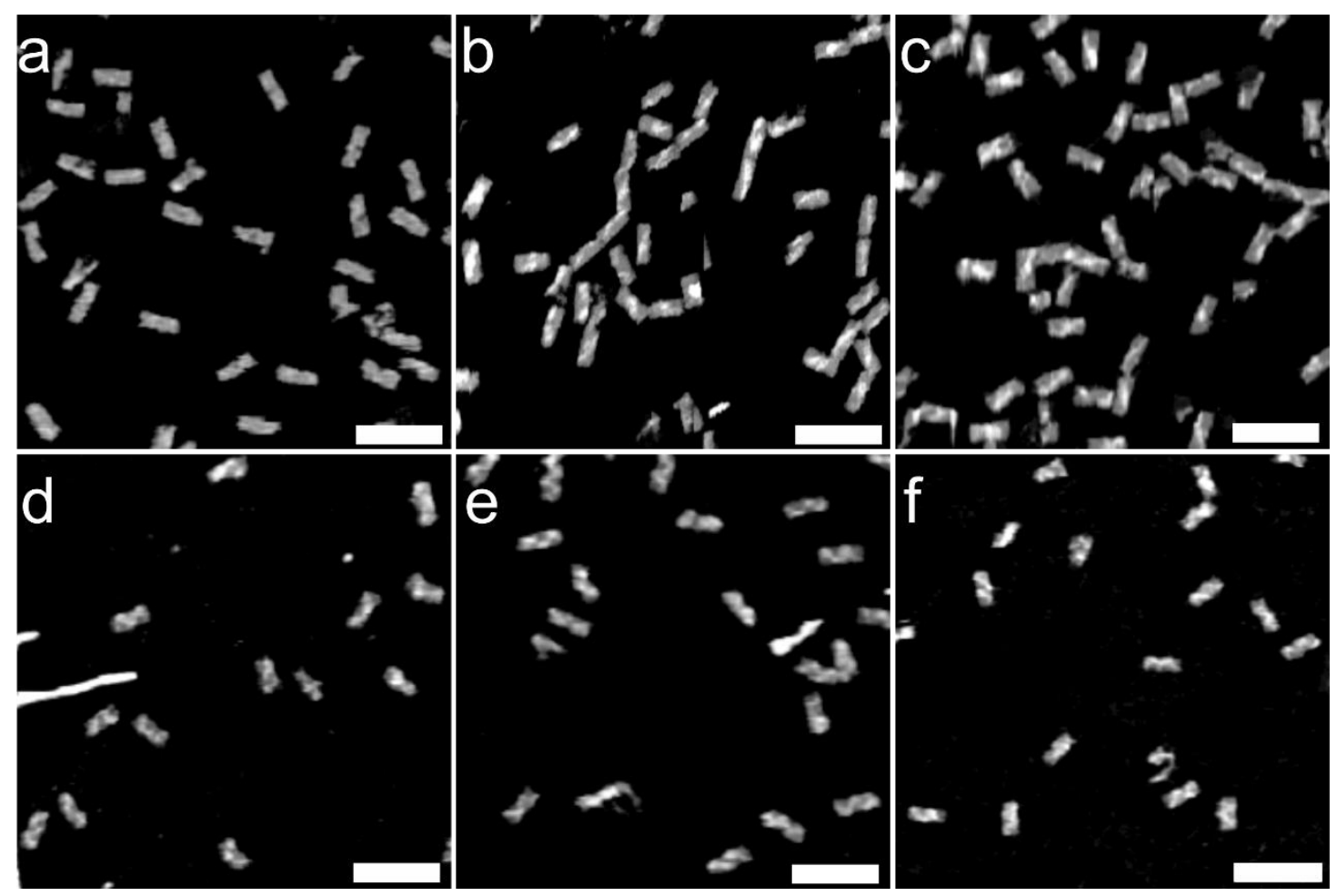

Figure S36. AFM images of helical silver patterns with varied configurations and pitches. (a) Unmetallized DNA origami with pcDNA. (b) Right-handed helices with a pitch of $100 \mathrm{~nm}$. (c) Left-handed helices with a pitch of $100 \mathrm{~nm}$. (d) Achiral patterns. (e) Right-handed helices with a pitch of $50 \mathrm{~nm}$. (f) Left-handed helices with a pitch of $50 \mathrm{~nm}$. Scale bar: $200 \mathrm{~nm}$. 


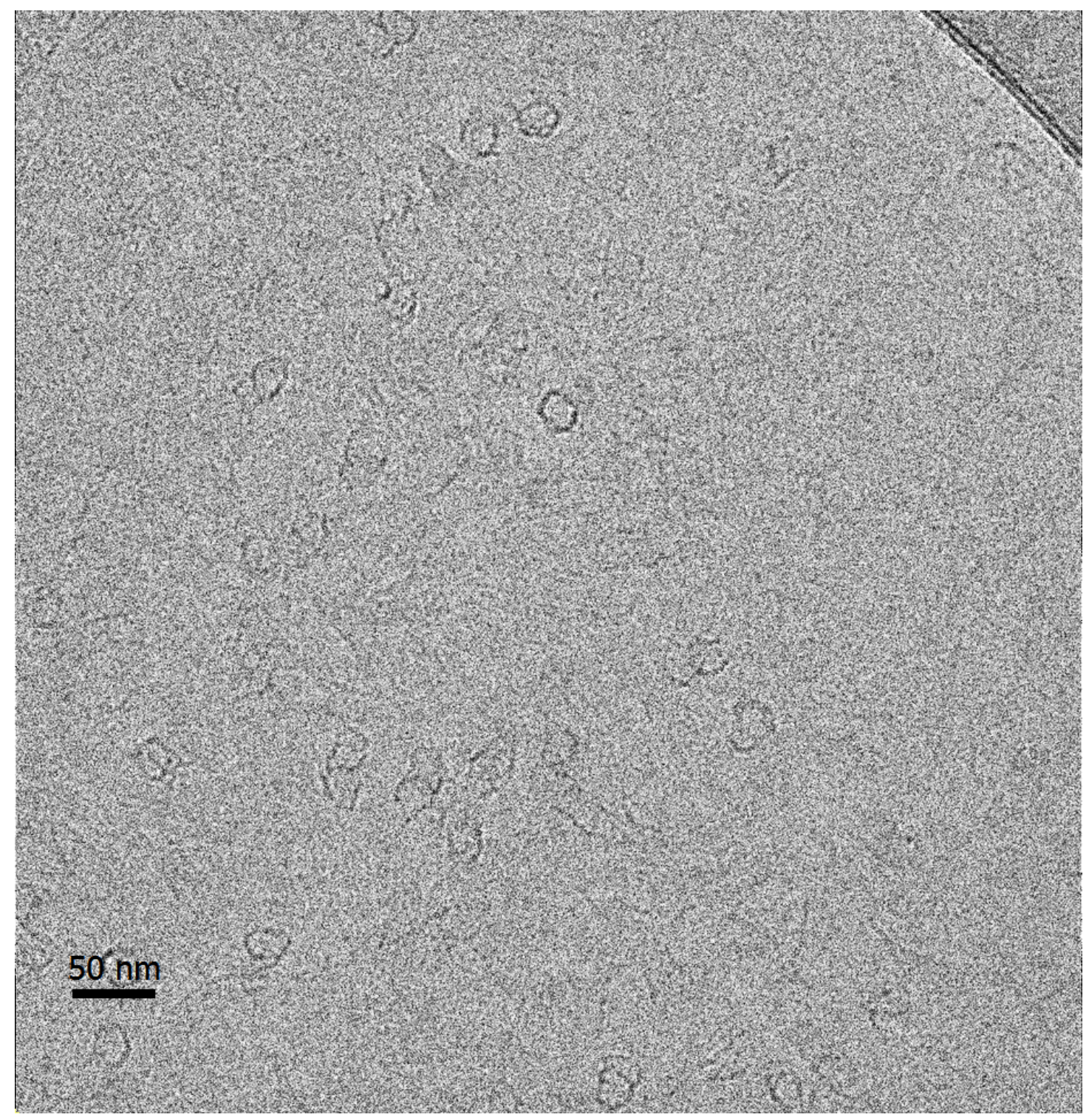

Figure S37. Cryo-EM image of helical silver patterns. The helical pitch is $100 \mathrm{~nm}$. 


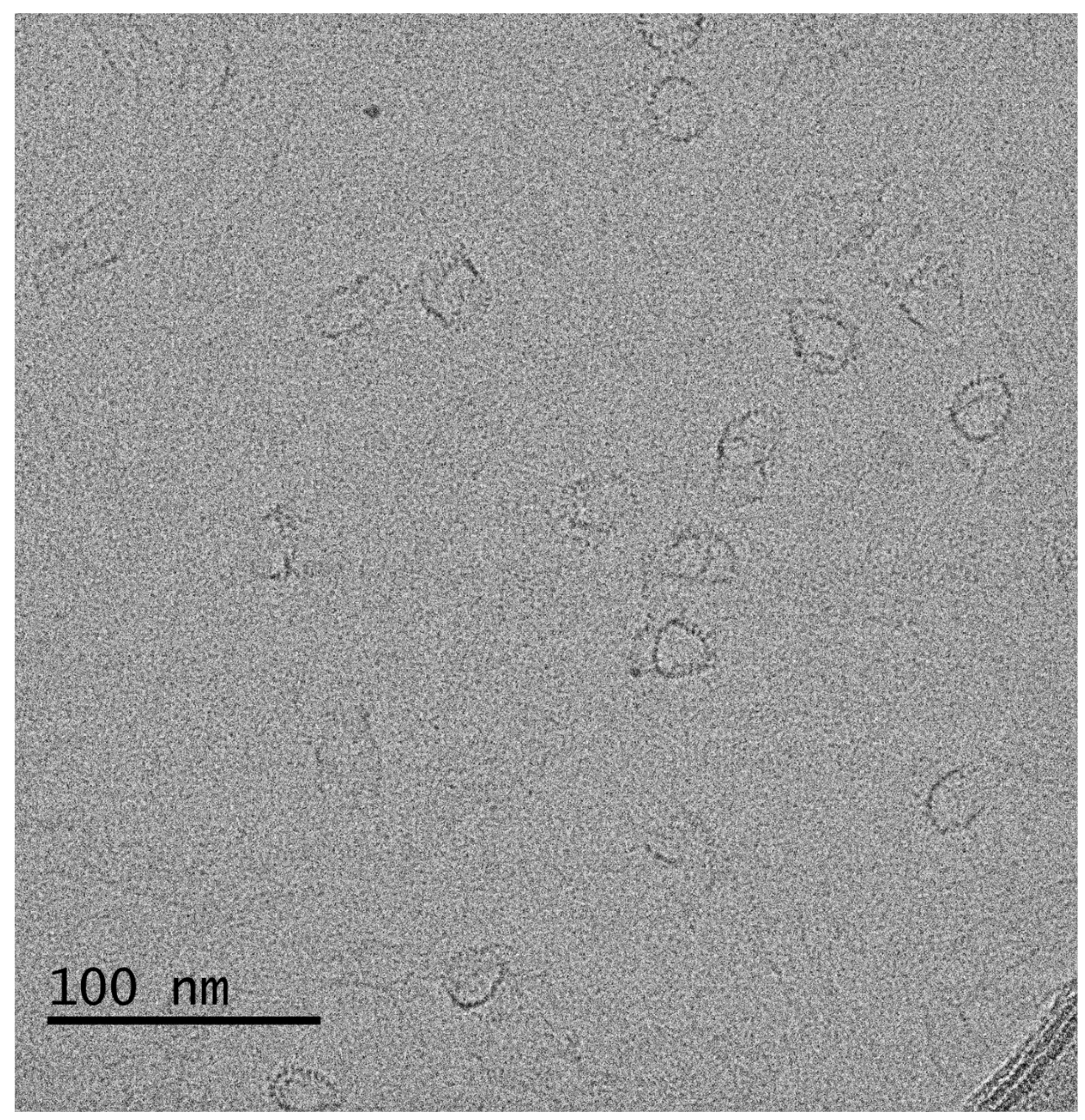

Figure S38. Magnified view of the helical silver patterns in cryo-EM imaging. 


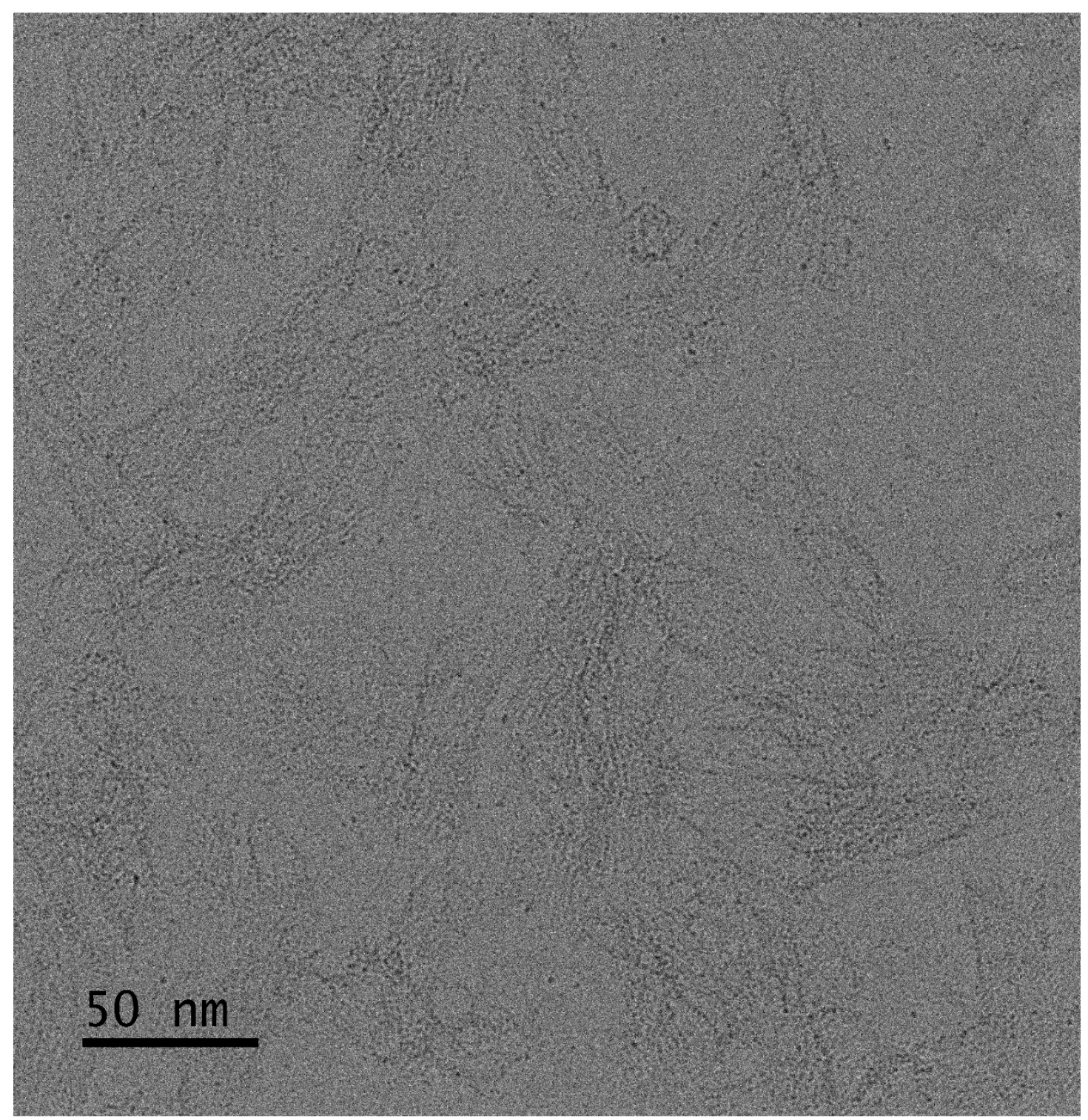

Figure S39. Unmetallized tubular DNA origami carrying pcDNA blueprint in cryo-EM imaging. 


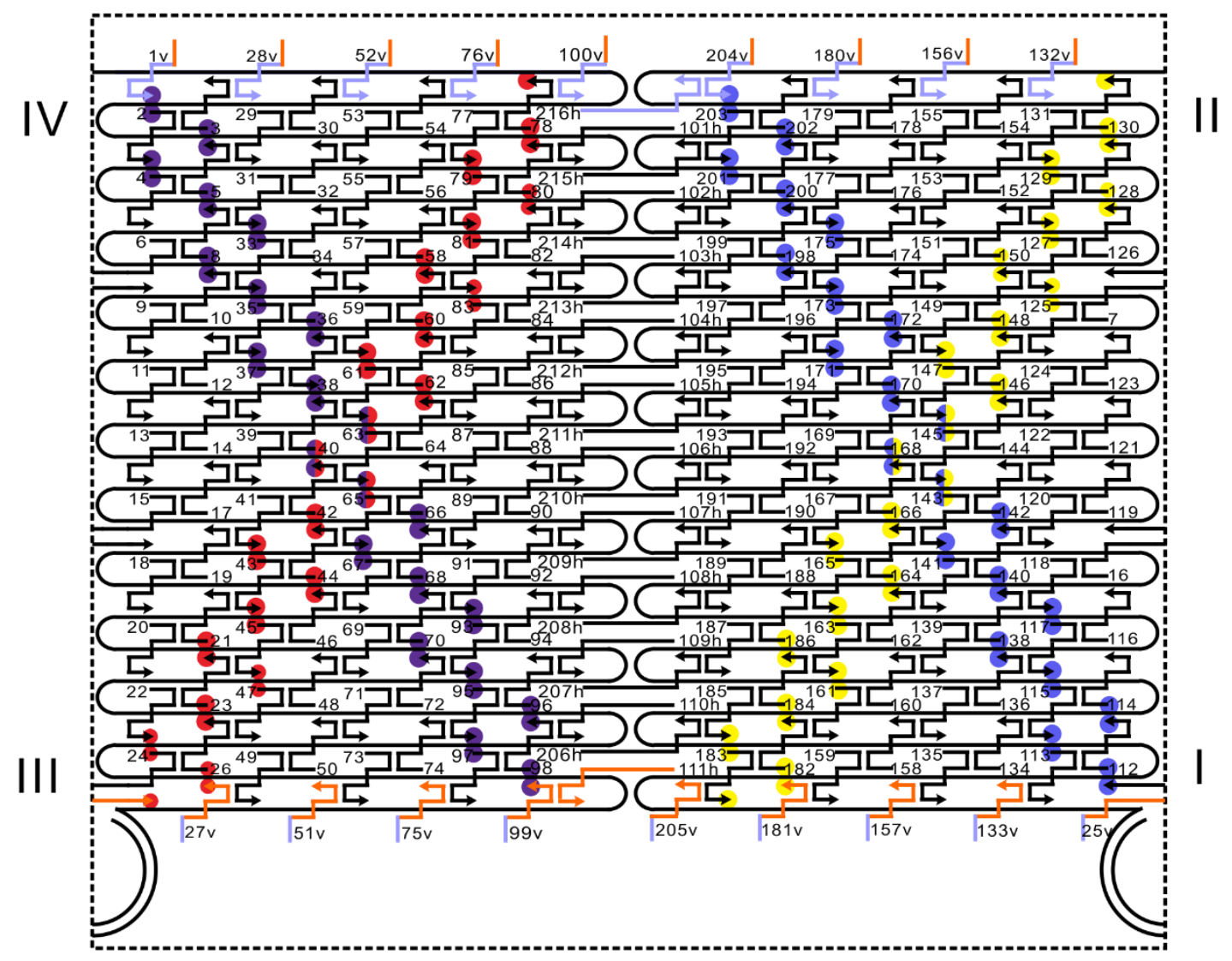

Figure S40. Schematic design of inter-assembly of DNA origami monomers. 


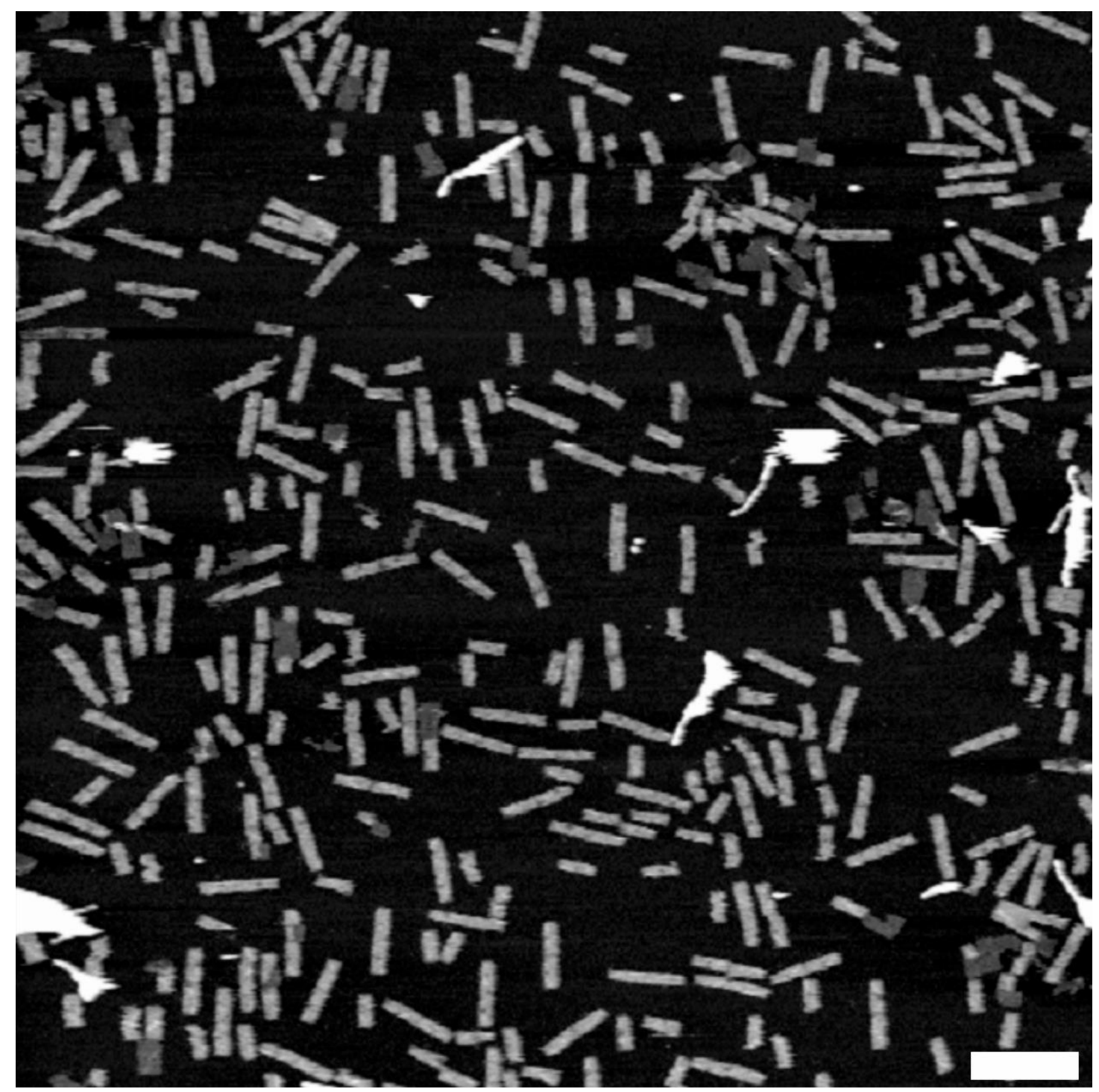

Figure S41. AFM image of tubular DNA origami dimers. Scale bar: $200 \mathrm{~nm}$. 

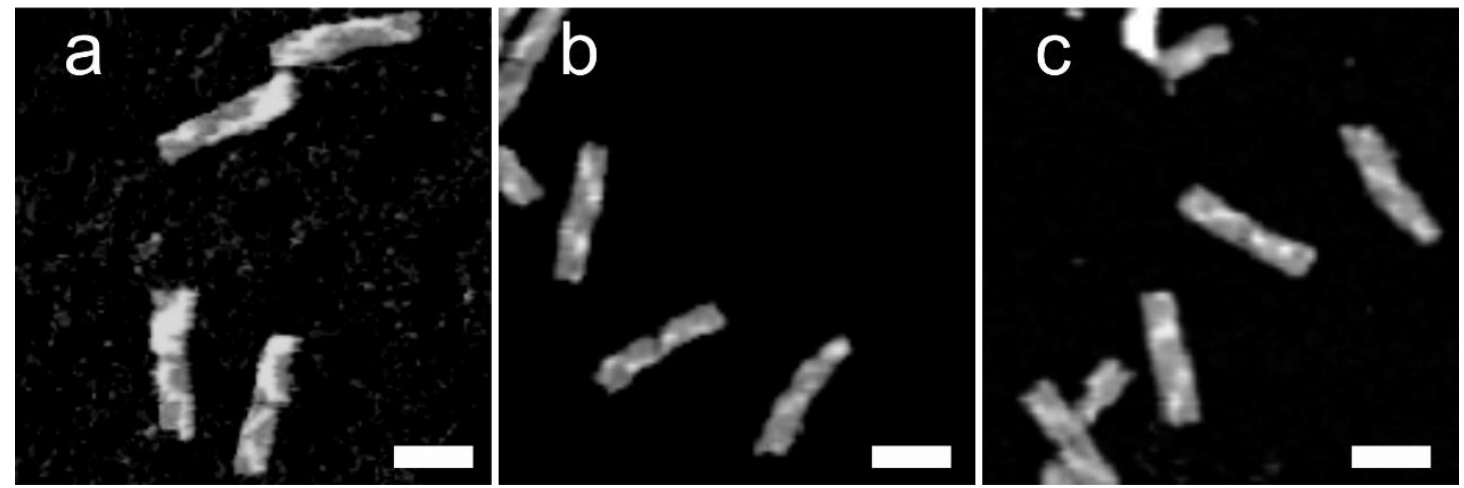

Figure S42. AFM images of helical silver patterns on tubular DNA origami dimers. (a) Achiral patterns. (b) Left-handed helices containing two pitches of $100 \mathrm{~nm}$. (c) Righthanded helices containing two pitches of $100 \mathrm{~nm}$. Scale bar: $100 \mathrm{~nm}$. 


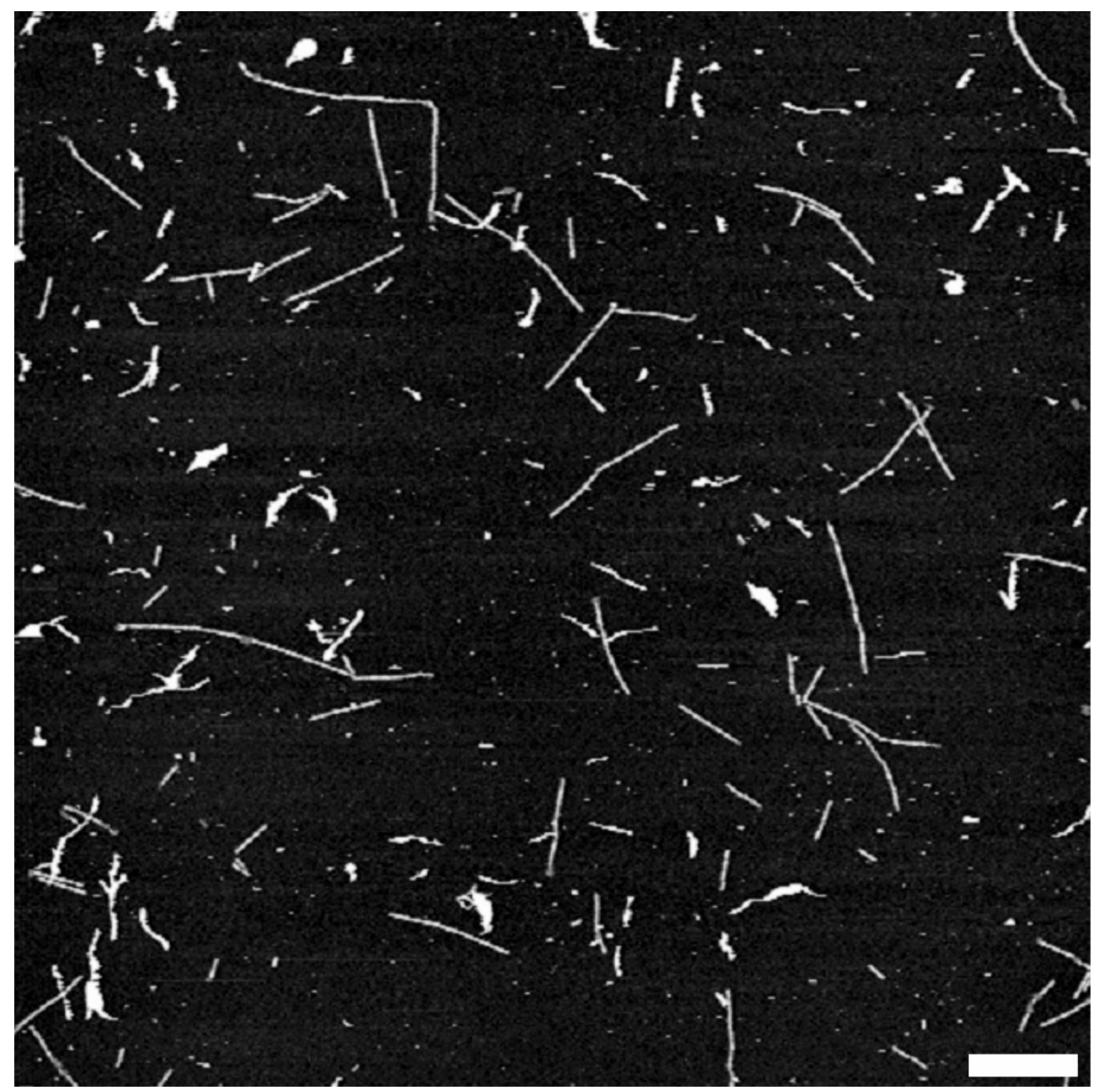

Figure S43. AFM image of DNA filaments with a length of up to $4 \mu \mathrm{m}$. Scale bar: 1 $\mu \mathrm{m}$. 


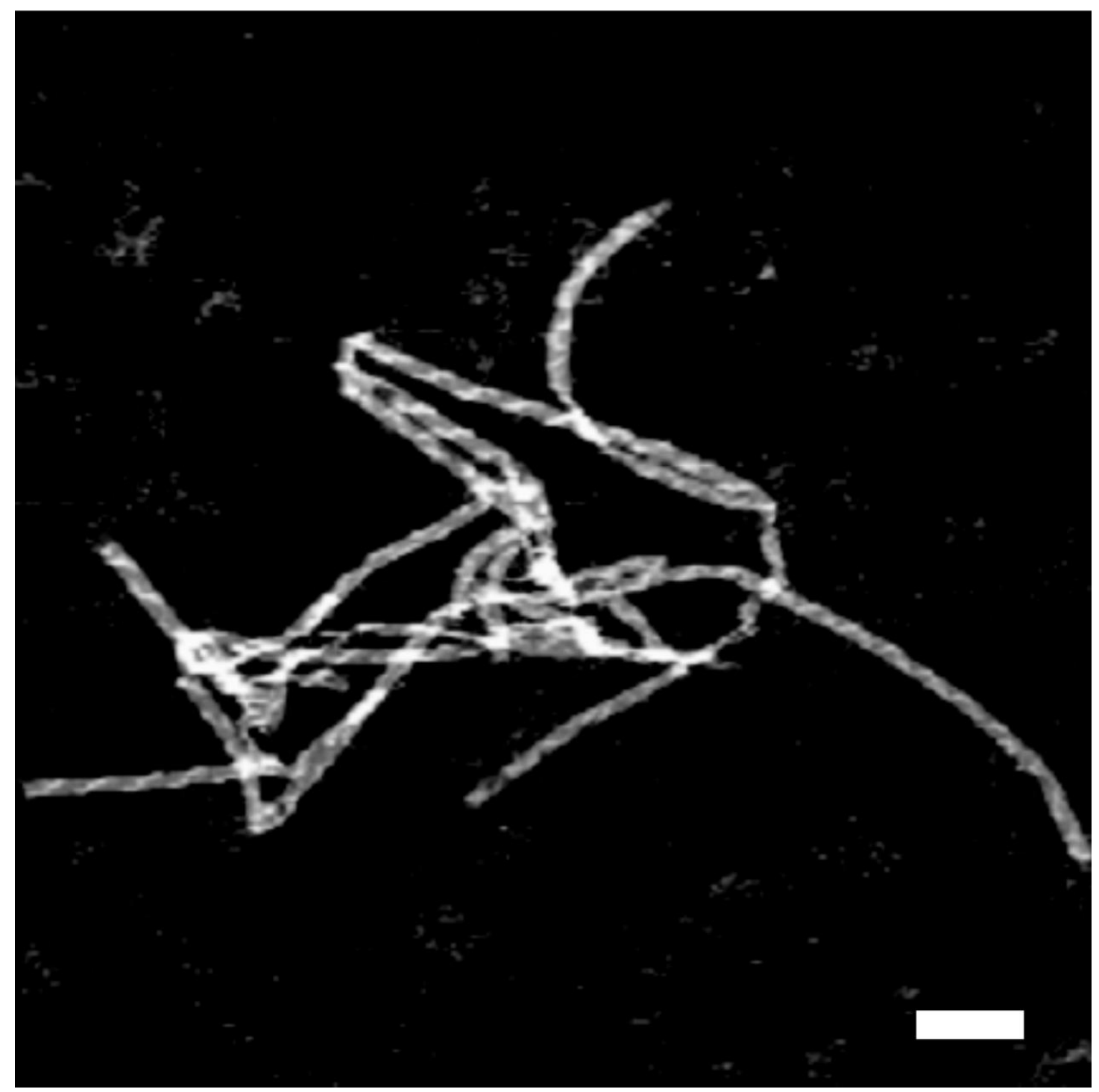

Figure S44. AFM image of left-handed silver helices with a pitch of $100 \mathrm{~nm}$ on DNA origami filaments. Scale bar: $200 \mathrm{~nm}$. 


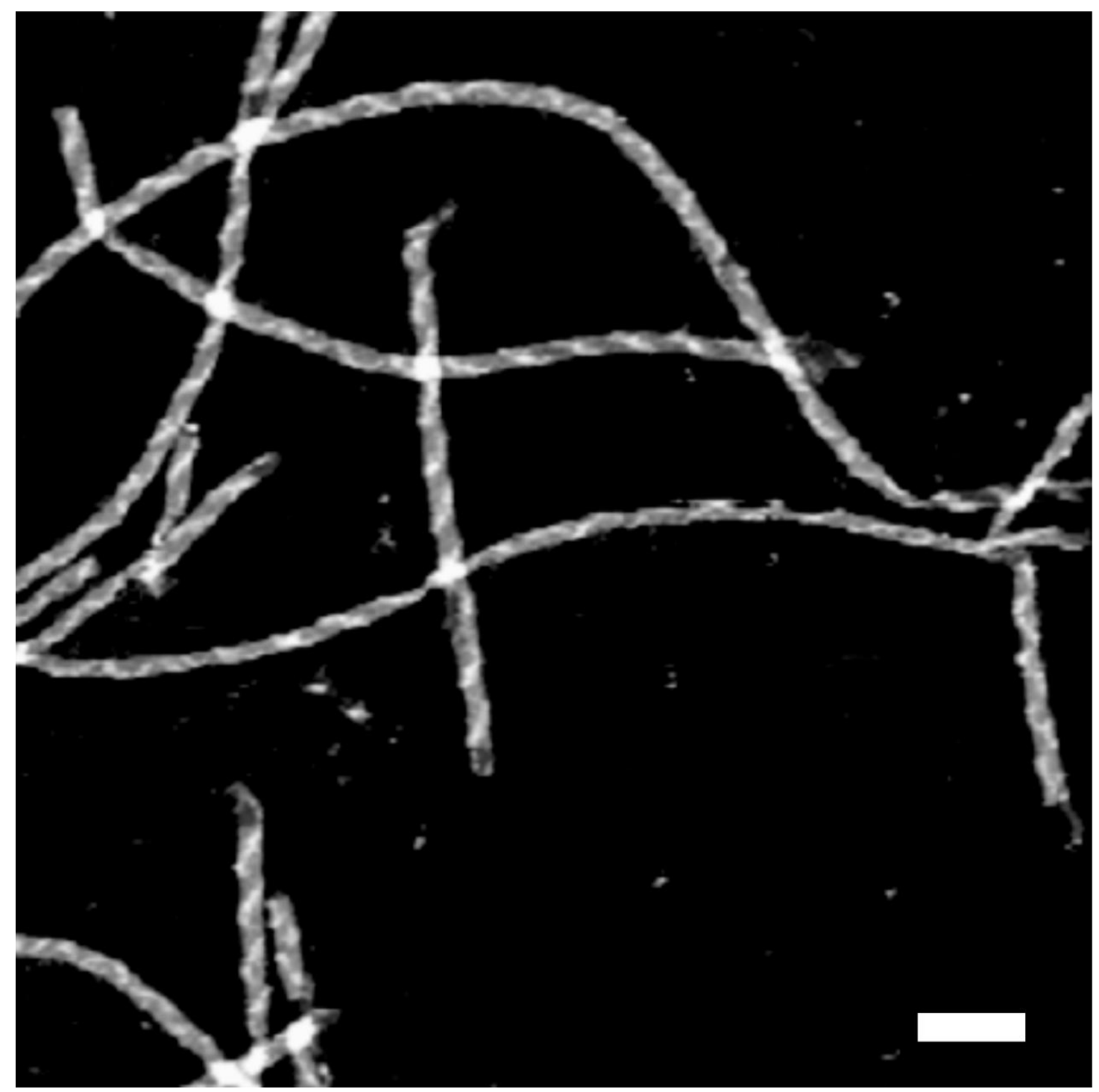

Figure S45. AFM image of right-handed silver helices with a pitch of $100 \mathrm{~nm}$ on DNA origami filaments. Scale bar: $200 \mathrm{~nm}$. 


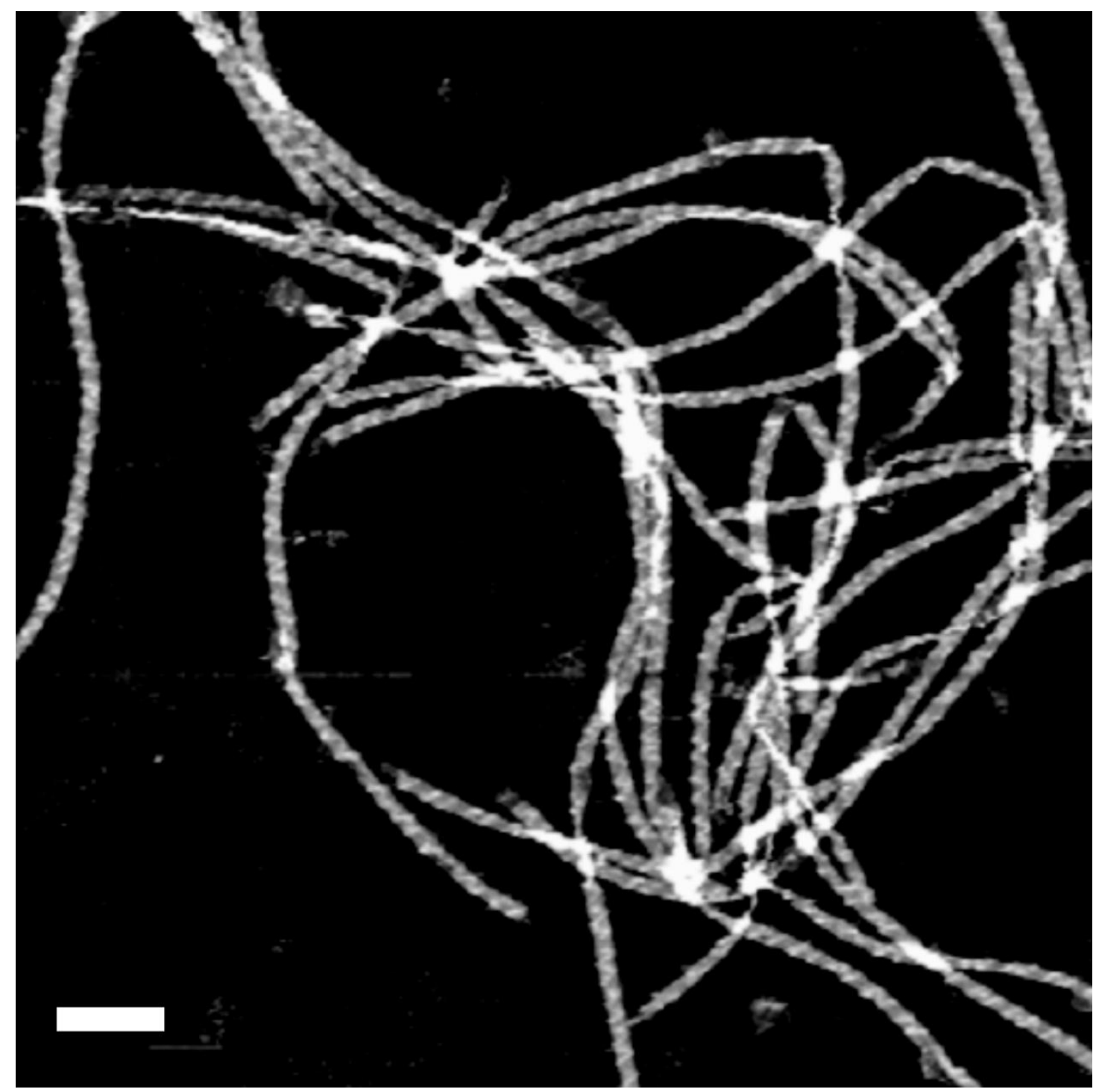

Figure S46. AFM image of left-handed silver helices with a pitch of $50 \mathrm{~nm}$ on DNA origami filaments. Scale bar: $200 \mathrm{~nm}$. 


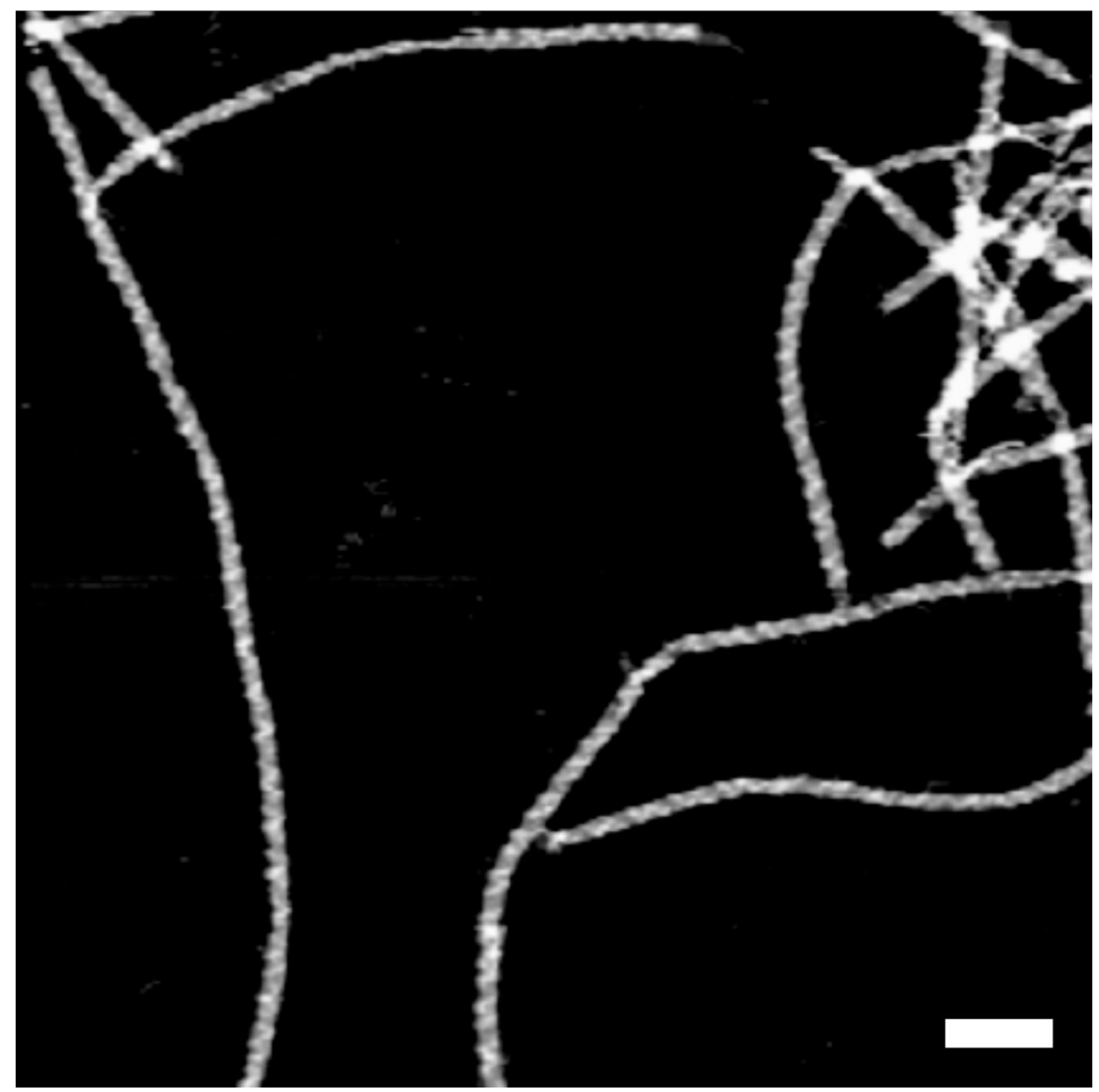

Figure S47. AFM image of right-handed silver helices with a pitch of $50 \mathrm{~nm}$ on DNA origami filaments. Scale bar: $200 \mathrm{~nm}$. 


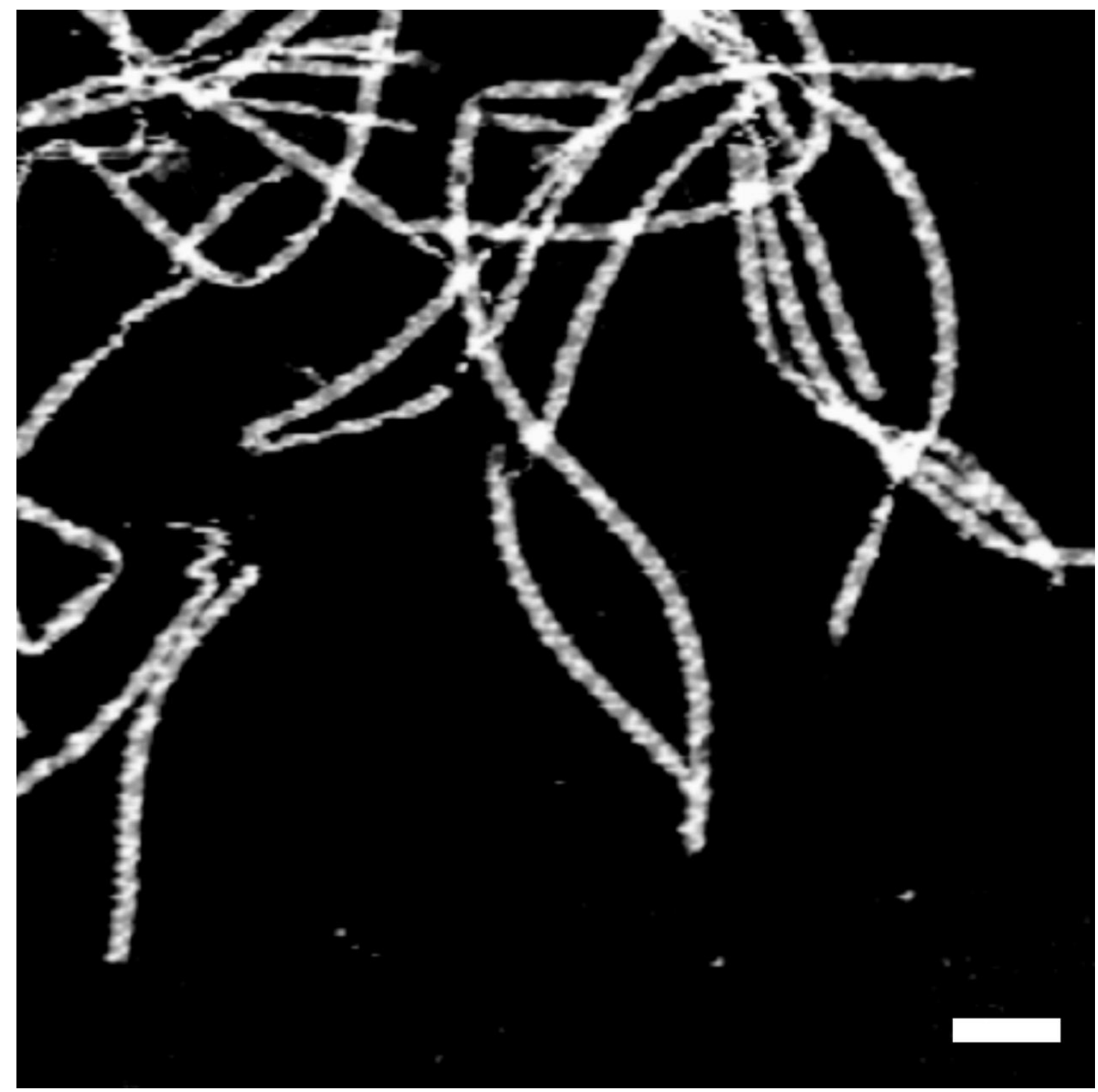

Figure S48. AFM image of achiral silver helices on DNA origami filaments. Scale bar: $200 \mathrm{~nm}$. 

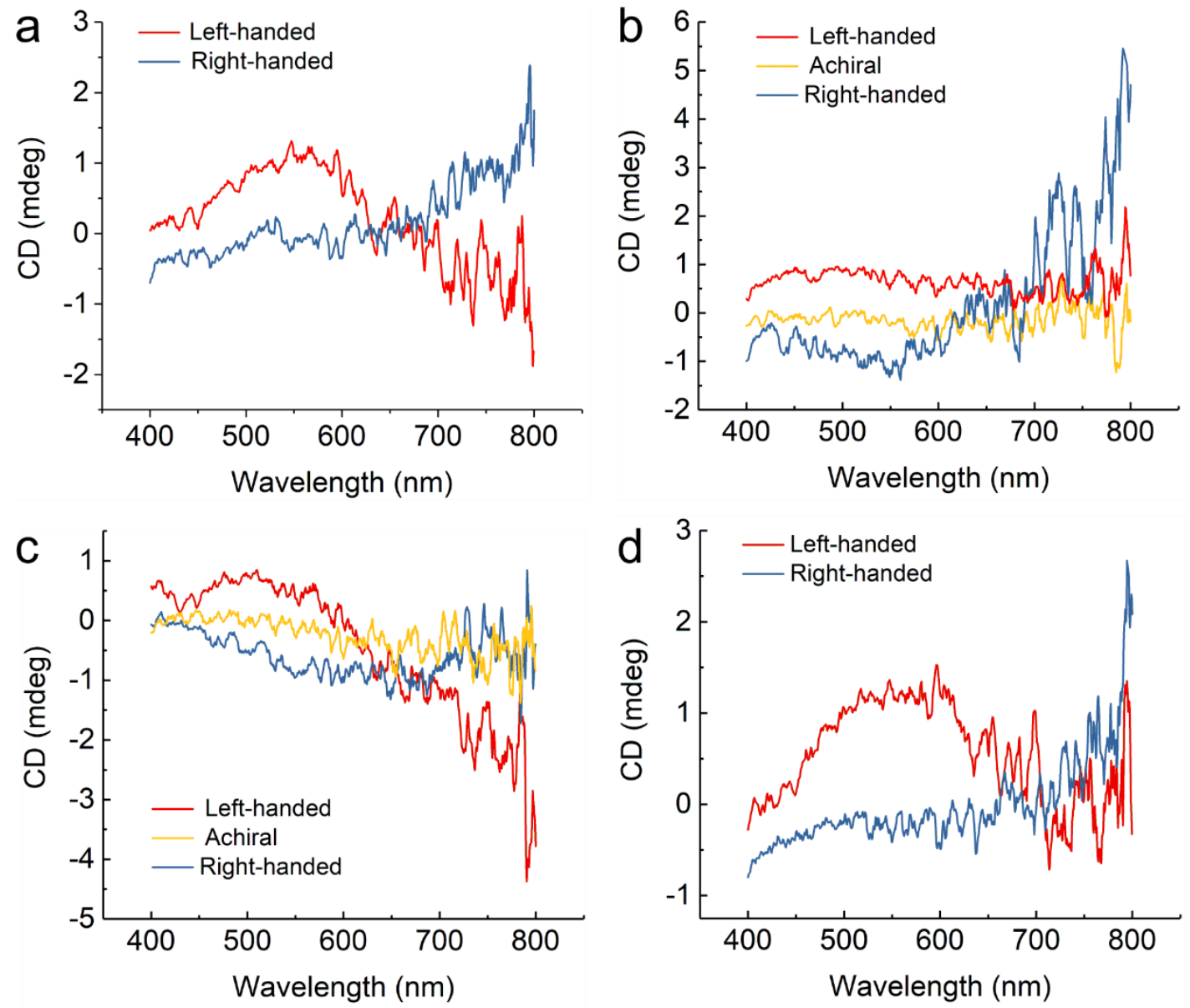

Figure S49. CD spectra of helical silver patterns on tubular osDNA. Two lines of 20nt pcDNA protrusions were used as the blueprint. (a) CD spectra of silver pattens with a helical pitch of $100 \mathrm{~nm}$. (b) The helical pitch is $50 \mathrm{~nm}$. (c) CD spectra of silver patterns on origami dimers. Pitch: $100 \mathrm{~nm}$. (d) CD spectra of silver patterns on 1D filament. Pitch: $100 \mathrm{~nm}$. The achiral pattern was made by joining two helical patterns of adverse chirality. 


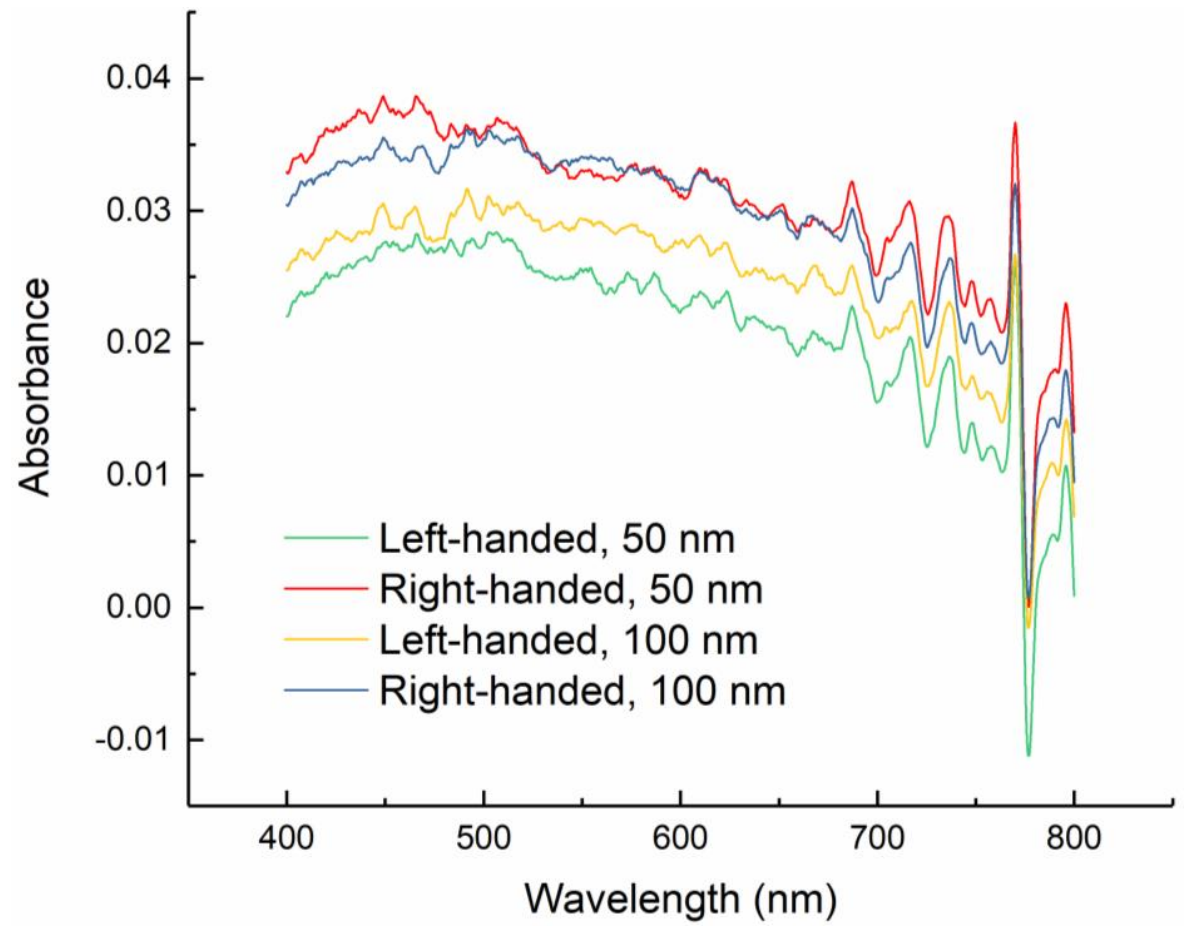

Figure S50. UV-vis absorption spectra of helical silver patterns. 


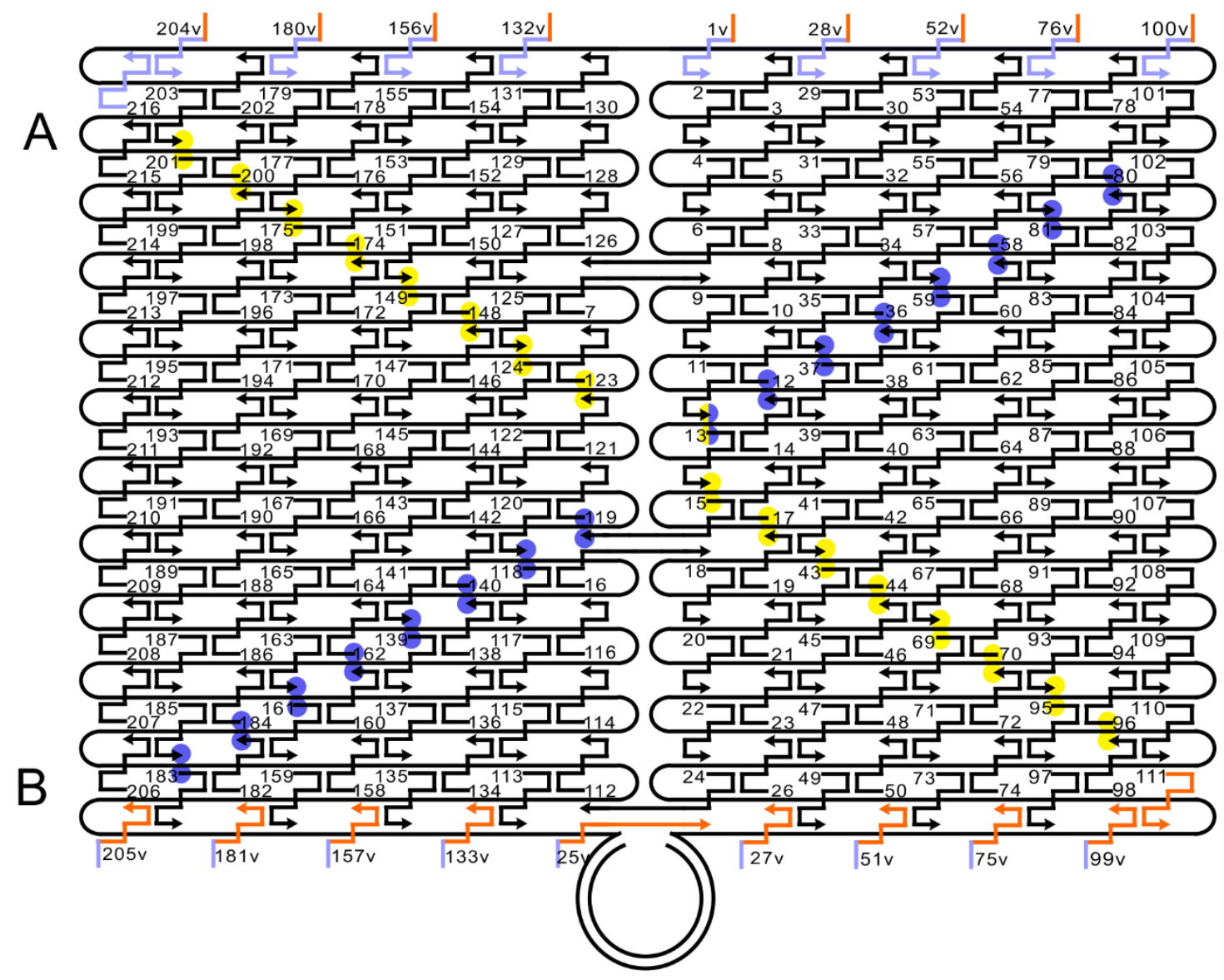

Figure S51. Schematic design of helical pcDNA templates with a pitch of $100 \mathrm{~nm}$ on DNA origami. Only a single line of pcDNA is presented in the protrusion. Eight bases were exchanged between the staples at the bottom and top of the DNA origami to roll the rectangle into a tube. 

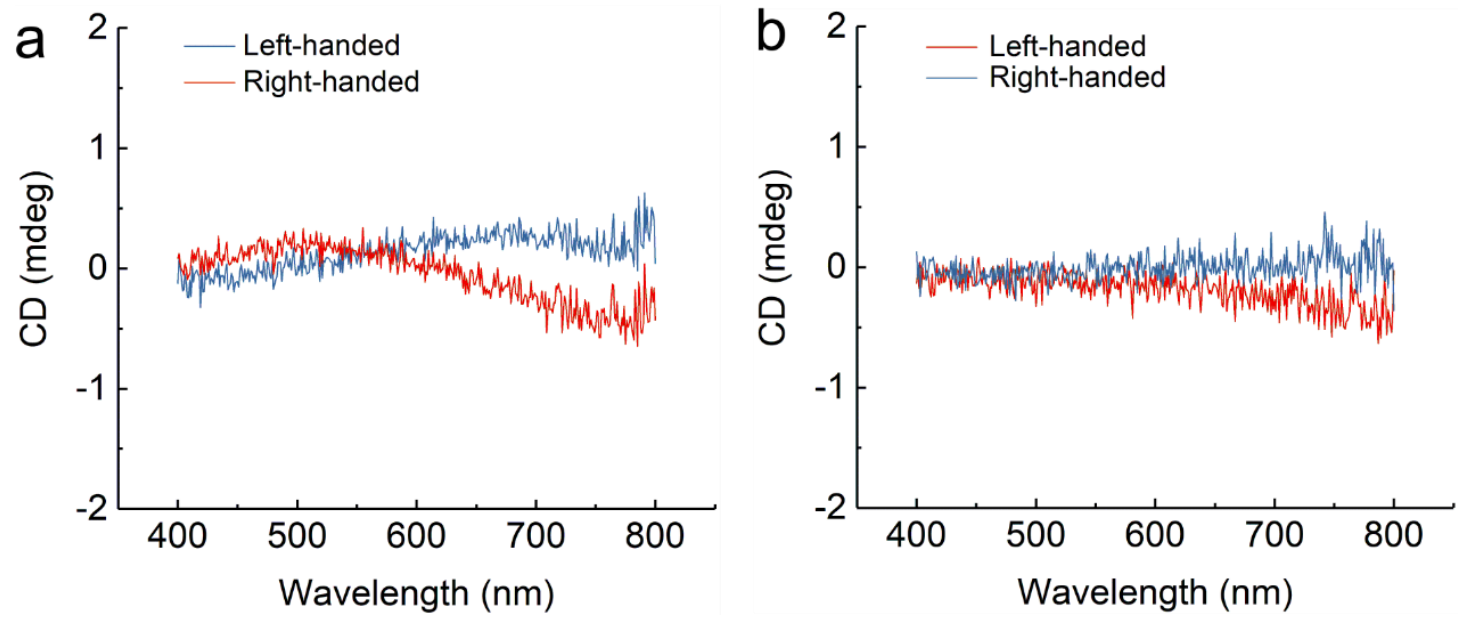

Figure S52. CD spectra of helical silver patterns on tubular osDNA for exploring the effect of thickness and continuity. (a) One line of 20-nt pcDNA protrusions was used. (b) Two lines of 10-nt pcDNA protrusions were used. Helical pitch: $100 \mathrm{~nm}$. 


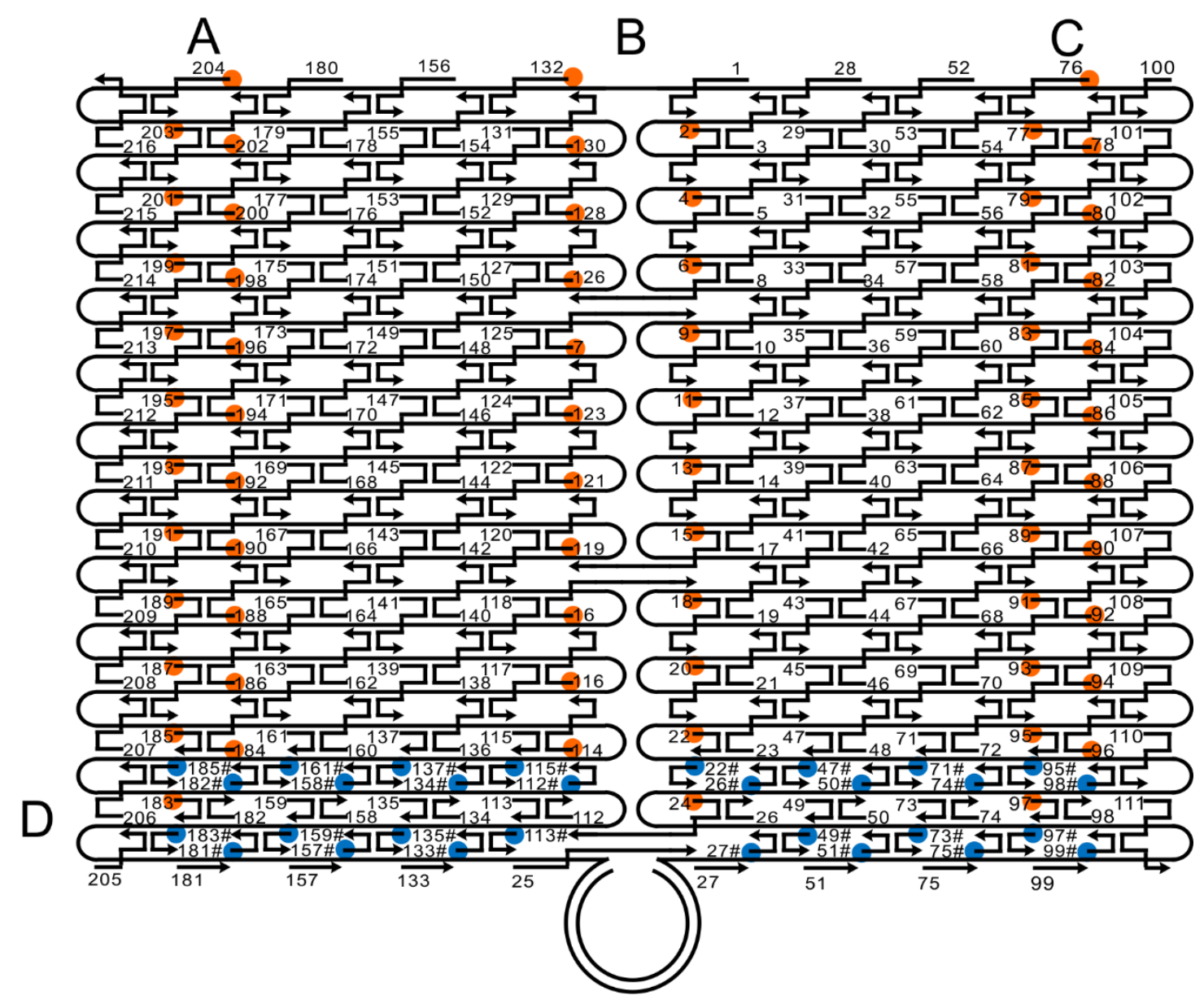

Figure S53. Schematic design of bifacial pcDNA templates on rectangular DNA origami. 


\section{DNA sequence}

Sequences of the other staples in rectangular and triangular DNA origami can be found in Ref. 5 and Ref. 36 in the main text, respectively.

\section{For the "8"-shaped pcDNA template on rectangular DNA origami}

199 GCGACGACCTGACGACATAGCTTTGAAAAGAACTGGCTCATTATTTAATAAA 197 GCGACGACCTGACGACATAGACGAACTAGCGTCCAATACTGCGGAATGCTTT 195 GCGACGACCTGACGACATAGAAACAGTTGATGGCTTAGAGCTTATTTAAATA 193 GCGACGACCTGACGACATAGTGCAACTAAGCAATAAAGCCTCAGTTATGACC 191 GCGACGACCTGACGACATAGCTGTAATATTGCCTGAGAGTCTGGAAAACTAG 189 GCGACGACCTGACGACATAGCATGTCAAGATTCTCCGTGGGAACCGTTGGTG 200 GCGACGACCTGACGACATAGGCTCCATGAGAGGCTTTGAGGACTAGGGAGTT 198 GCGACGACCTGACGACATAGCGATTTTAGAGGACAGATGAACGGCGCGACCT 196 GCGACGACCTGACGACATAGACTGGATAACGGAACAACATTATTACCTTATG 194 GCGACGACCTGACGACATAGTTTTTGCGCAGAAAACGAGAATGAATGTTTAG 192 GCGACGACCTGACGACATAGCAAAATTAAAGTACGGTGTCTGGAAGAGGTCA 190 GCGACGACCTGACGACATAGTCAGGTCACTTTTGCGGGAGAAGCAGAATTAG 188 GCGACGACCTGACGACATAGACCCGTCGTCATATGTACCCCGGTAAAGGCTA 177 GCGACGACCTGACGACATAGTTTCATGAAAATTGTGTCGAAATCTGTACAGA 163 GCGACGACCTGACGACATAGGTTTGAGGGAAAGGGGGATGTGCTAGAGGATC 178 GCGACGACCTGACGACATAGATATATTCTTTTTTCACGTTGAAAATAGTTAG 176 GCGACGACCTGACGACATAGCGCCTGATGGAAGTTTCCATTAAACATAACCG 164 GCGACGACCTGACGACATAGCTTTCATCCCCAAAAACAGGAAGACCGGAGAG 162 GCGACGACCTGACGACATAGCAGCTGGCGGACGACGACAGTATCGTAGCCAG 153 GCGACGACCTGACGACATAGATACGTAAAAGTACAACGGAGATTTCATCAAG 139 GCGACGACCTGACGACATAGGAAGATCGGTGCGGGCCTCTTCGCAATCATGG 154 GCGACGACCTGACGACATAGCAATGACACTCCAAAAGGAGCCTTACAACGCC 152 GCGACGACCTGACGACATAGGCGAAACATGCCACTACGAAGGCATGCGCCGA 140 GCGACGACCTGACGACATAGAAATAATTTTAAATTGTAAACGTTGATATTCA 138 GCGACGACCTGACGACATAGGGCGATCGCACTCCAGCCAGCTTTGCCATCAA 129 GCGACGACCTGACGACATAGAAACGAAATGACCCCCAGCGATTATTCATTAC 117 GCGACGACCTGACGACATAGGCTTCTGGTCAGGCTGCGCAACTGTGTTATCC 130 GCGACGACCTGACGACATAGCTTAAACATCAGCTTGCTTTCGAGAAACAGTT 128 GCGACGACCTGACGACATAGCTCATCTTGAGGCAAAAGAATACACTCCCTCA 126 GCGACGACCTGACGACATAGGAATAAGGACGTAACAAAGCTGCTGACGGAAA 7 GCGACGACCTGACGACATAGCATAACCCGAGGCATAGTAAGAGCTTTTTAAG 123 GCGACGACCTGACGACATAGTTTTAATTGCCCGAAAGACTTCAATTCCAGAG 121 GCGACGACCTGACGACATAGTTTCATTTGGTCAATAACCTGTTTAATCAATA 119 GCGACGACCTGACGACATAGAGACAGTCATTCAAAAGGGTGAGATATCATAT 16 GCGACGACCTGACGACATAGGCTCATTTTCGCATTAAATTTTTGAGCTTAGA 116 GCGACGACCTGACGACATAGTTCGCCATTGCCGGAAACCAGGCAAACAGTAC 4 GCGACGACCTGACGACATAGGAGCCGCCCCACCACCGGAACCGCCTAAAACA 6 GCGACGACCTGACGACATAGTTATTCATAGGGAAGGTAAATATTCATTCAGT 
9 GCGACGACCTGACGACATAGAAAAGTAATATCTTACCGAAGCCCAACACTAT 11 GCGACGACCTGACGACATAGCCTAATTTACGCTAACGAGCGTCTATATCGCG 13 GCGACGACCTGACGACATAGATCGGCTGCGAGCATGTAGAAACCAGCTATAT 15 GCGACGACCTGACGACATAGGCGTTATAGAAAAAGCCTGTTTAGAAGGCCGG 18 GCGACGACCTGACGACATAGTTAAGACGTTGAAAACATAGCGATTTAAATCA 20 GCGACGACCTGACGACATAGCTTTTACACAGATGAATATACAGTAAGCGCCA 3 GCGACGACCTGACGACATAGTGCCTTGACTGCCTATTTCGGAACAGGGATAG 5 GCGACGACCTGACGACATAGAACCAGAGACCCTCAGAACCGCCAGGGGTCAG 19 GCGACGACCTGACGACATAGTAGAATCCCTGAGAAGAGTCAATAGGAATCAT 21 GCGACGACCTGACGACATAGTTTAACGTTCGGGAGAAACAATAATTTTCCCT 31 GCGACGACCTGACGACATAGGCCACCACTCTTTTCATAATCAAACCGTCACC 45 GCGACGACCTGACGACATAGCCTGATTGAAAGAAATTGCGTAGACCCGAACG 30 GCGACGACCTGACGACATAGAGTGTACTTGAAAGTATTAAGAGGCCGCCACC 32 GCGACGACCTGACGACATAGGTTTGCCACCTCAGAGCCGCCACCGATACAGG 44 GCGACGACCTGACGACATAGCTGTAAATCATAGGTCTGAGAGACGATAAATA 46 GCGACGACCTGACGACATAGACAGAAATCTTTGAATACCAAGTTCCTTGCTT32 55 GCGACGACCTGACGACATAGCACCAGAGTTCGGTCATAGCCCCCGCCAGCAA 69 GCGACGACCTGACGACATAGGCGCAGAGATATCAAAATTATTTGACATTATC 54 GCGACGACCTGACGACATAGTAAGCGTCGAAGGATTAGGATTAGTACCGCCA 56 GCGACGACCTGACGACATAGTCGGCATTCCGCCGCCAGCATTGACGTTCCAG 68 GCGACGACCTGACGACATAGAAATCAATGGCTTAGGTTGGGTTACTAAATTT 70 GCGACGACCTGACGACATAGAACCTACCGCGAATTATTCATTTCCAGTACAT 79 GCGACGACCTGACGACATAGTGAGGCAGGCGTCAGACTGTAGCGTAGCAAGG 81 GCGACGACCTGA CGACATAGCCGGAAACACACCACGGAATAAGTAAGACTCC 83 GCGACGACCTGACGACATAGTTATTACGGTCAGAGGGTAATTGAATAGCAGC 85 GCGACGACCTGACGACATAGCTTTACAGTTAGCGAACCTCCCGACGTAGGAA 87 GCGACGACCTGACGACATAGTCATTACCCGACAATAAACAACATATTTAGGC 89 GCGACGACCTGACGACATAGAGAGGCATAATTTCATCTTCTGACTATAACTA 91 GCGACGACCTGACGACATAGTATGTAAACCTTTTTTAATGGAAAAATTACCT 93 GCGACGACCTGACGACATAGGAGCAAAAACTTCTGAATAATGGAAGAAGGAG 80 GCGACGACCTGACGACATAGTGCCTTTAGTCAGACGATTGGCCTGCCAGAAT 82 GCGACGACCTGACGACATAGACGCAAAGGTCACCAATGAAACCAATCAAGTT 84 GCGACGACCTGACGACATAGTGAACAAACAGTATGTTAGCAAACTAAAAGAA 86 GCGACGACCTGACGACATAGGAGGCGTTAGAGAATAACATAAAAGAACACCC 88 GCGACGACCTGACGACATAGCCAGACGAGCGCCCAATAGCAAGCAAGAACGC 90 GCGACGACCTGACGACATAGTTTTAGTTTTTCGAGCCAGTAATAAATTCTGT 92 GCGACGACCTGACGACATAGTTGAATTATGCTGATGCAAATCCACAAATATA

\section{For helical pcDNA templates on tubular DNA origami \\ Staples used in DNA origami tubulation}

1v CGGCCTTGATAGGAACCCATGTACAAACAGTT

$1 \mathrm{v}-\mathrm{IV}$

CGGCCTTGATAGGAACCCATGTACAAACAGTTGCGACGACCTGACGACATAG 25v TGAGTTTCCGAGAAAGGAAGGGAACAAACTAT 
$25 \mathrm{v}-$ III

TGAGTTTCCGAGAAAGGAAGGGAACAAACTATGCGACGACCTGACGACATAG

27v CAAGCCCACTGGTAATATCCAGAACGAACTGA

$27 \mathrm{v}-\mathrm{III}$

CAAGCCCACTGGTAATATCCAGAACGAACTGAGCGACGACCTGACGACATAG

28v CCGCCAGCCACCACCCTCATTTTCCTATTATT

51v CTCAGAGCCATTGCAACAGGAAAAATATTTTT

52v GGAAATACACCGCCACCCTCAGAACTGAGACT

75v CCCTCAGACTACATTTTGACGCTCACCTGAAA

76v GAAATGGATACTCAGGAGGTTTAGCGGGGTTT

99v TATCACCGTTATTTACATTGGCAGACATTCTG

99v-IV TATCACCGTTATTTACATTGGCAGACATTCTGGCGACGACCTGACGACATAG

100v GTCACACGTATAAGTATAGCCCGGCCGTCGAG

132v GAACGTGGGTCACCAGTACAAACTTAATTGTA

133v TGTAGCATTAGAGCTTGACGGGGAAATCAAAA

156v CCCCGATTTCCACAGACAGCCCTCATCTCCAA

157v CGTAACGACTAAATCGGAACCCTAGTTGTTCC

180v GTAAAGCATCTAAAGTTTTGTCGTGAATTGCG

181v ACGTTAGTCAAGTTTTTTGGGGTCAAAGAACG

$181 \mathrm{v}-\mathrm{II}$

ACGTTAGTCAAGTTTTTTGGGGTCAAAGAACGGCGACGACCTGACGACATAG

204v ACCCAAATAAATGAATTTTCTGTAAGCGGAGT

204v-I

ACCCAAATAAATGAATTTTCTGTAAGCGGAGTGCGACGACCTGACGACATAG

205v TGCTAAACCGATGGCCCACTACGTAAACCGTC

$204 \mathrm{v}-\mathrm{I}$ was used instead of 204v when pcDNA was along I line. 181v-II was used instead of $181 \mathrm{v}$ when pcDNA was along II line. $25 \mathrm{v}$-III and $27 \mathrm{v}$-III were used instead of $25 \mathrm{v}$ and $27 \mathrm{v}$ when pcDNA was along III line. $1 \mathrm{v}-\mathrm{IV}$ was used instead of $1 \mathrm{v}$ when pcDNA was along IV line.

\section{Staples used in DNA origami polymerization}

101h CAGCGAAAATAAATCCTCATTAAATGATATTC

102h ACGGTCAAAATCAGTAGCGACAGATCGATAGC

103h GGACGTTGTAAAGGTGGCAACATAGTAGAAAA

104h TAAATATTGACGGGAGAATTAACTACAGGGAA

105h ATATAATGGCTTATCCGGTATTCTAAATCAGA

106h GCTAAATCCGACAAAAGGTAAAGTAGAGAATA

107h AGAGAATCCGCGAGAAAACTTTTTATCGCAAG

108h GATTGACCATTAATTACATTTAACACATCAAG

109h CACGACGTTTCATCAATATAATCCTATCAGAT

110h GGGAGAGGAATCAATATCTGGTCACAAATATC

111h TATCAGGGACCAGTAATAAAAGGGATTCACCA

206h AAACCCTCCGGTTTGCGTATTGGGAACGCGCG 
207h GATGGCAATGTAAAACGACGGCCATTCCCAGT

208h AAAACAAAGTAATGGGATAGGTCAAAACGGCG

209h ACAAAGAAGATGAACGGTAATCGTAGCAAACA

210h TAAAGTACGGTTGTACCAAAAACAAGCATAAA

211h TATAGAAGCTGTAGCTCAACATGTATTGCTGA

212h GCGCATTACATTGAATCCCCCTCAAATCGTCA

213h TACATACAGGAAGAAAAATCTACGACCAGTCA

214h AGCACCGTTCATAAGGGAACCGAAAGGCGCAG

215h ACAAACAAGACAGCATCGGAACGAACCCTCAG

216h AGGGTTGAAACTTTCAACAGTTTCTGGGATTT

\section{Elongated staples (100-nm pitch)}

203 GAGAATAGCTTTTGCGGGATCGTCGGGTAGCAGTCGCTCTCTCAAGTAGAAT 201

GCGACGACCTGACGACATAGACGGCTACTTACTTAGCCGGAACGCTGACCAAGTC GCTCTCTCAAGTAGAAT

199 GCGACGACCTGACGACATAGCTTTGAAAAGAACTGGCTCATTATTTAATAAA 200 GCGACGACCTGACGACATAGGCTCCATGAGAGGCTTTGAGGACTAGGGAGTT 196 ACTGGATAACGGAACAACATTATTACCTTATGGTCGCTCTCTCAAGTAGAAT 198

GCGACGACCTGACGACATAGCGATTTTAGAGGACAGATGAACGGCGCGACCTGTC GCTCTCTCAAGTAGAAT

177 TTTCATGAAAATTGTGTCGAAATCTGTACAGAGTCGCTCTCTCAAGTAGAAT 175

GCGACGACCTGACGACATAGCCAGGCGCTTAATCATTGTGAATTACAGGTAGGTCG CTCTCTCAAGTAGAAT

173 GCGACGACCTGACGACATAGAAAGATTCAGGGGGTAATAGTAAACCATAAAT 174 GCGACGACCTGACGACATAGTTTCAACTATAGGCTGGCTGACCTTGTATCAT 172

GCGACGACCTGACGACATAGTTTGCCAGATCAGTTGAGATTTAGTGGTTTAAGTCG CTCTCTCAAGTAGAAT

170 TACCTTTAAGGTCTTTACCCTGACAAAGAAGTGTCGCTCTCTCAAGTAGAAT 151 AGTAATCTTAAATTGGGCTTGAGAGAATACCAGTCGCTCTCTCAAGTAGAAT 149

GCGACGACCTGACGACATAGCATTCAACGCGAGAGGCTTTTGCATATTATAGGTCG CTCTCTCAAGTAGAAT 147 GCGACGACCTGACGACATAGTCAGAAGCCTCCAACAGGTCAGGATCTGCGAA 148 GCGACGACCTGACGACATAGCCAAAATATAATGCAGATACATAAACACCAGA 146

GCGACGACCTGACGACATAGGAAGCAAAAAAGCGGATTGCATCAGATAAAAAGTC GCTCTCTCAAGTAGAAT

144 TCAATTCTTTTAGTTTGACCATTACCAGACCGGTCGCTCTCTCAAGTAGAAT 125 GGAATTACTCGTTTACCAGACGACAAAAGATTGTCGCTCTCTCAAGTAGAAT 124 
GCGACGACCTGACGACATAGAAGAGGAACGAGCTTCAAAGCGAAGATACATTGTC GCTCTCTCAAGTAGAAT

122A GCGACGACCTGACGACATAGTCGCAAATGGGGCGCGAGCTGAAATAATGTGT 122B TCGCAAATGGGGCGCGAGCTGAAATAATGTGTGTCGCTCTCTCAAGTAGAAT 123 GCGACGACCTGACGACATAGTTTTAATTGCCCGAAAGACTTCAAAACACTAT $121 \mathrm{~A}$

GCGACGACCTGACGACATAGTTTCATTTGGTCAATAACCTGTTTATATCGCGGTCGC TCTCTCAAGTAGAAT

121B GTCGCTCTCTCAAGTAGAATTTTCATTTGGTCAATA ACCTGTTTATATCGCG 119A AGACAGTCATTCAAAAGGGTGAGAAGCTATATGTCGCTCTCTCAAGTAGAAT 119B

GTCGCTCTCTCAAGTAGAATAGACAGTCATTCAAAAGGGTGAGAAGCTATATGCGA CGACCTGACGACATAG

11A CCTAATTTACGCTAACGAGCGTCTAATCAATAGTCGCTCTCTCAAGTAGAAT $11 \mathrm{~B}$

GTCGCTCTCTCAAGTAGAATCCTAATTTACGCTAACGAGCGTCTAATCAATAGCGAC GACCTGACGACATAG

14A GCGACGACCTGACGACATAGCTAATTTATCTTTCCTTATCATTCATCCTGAA 14B CTAATTTATCTTTCCTTATCATTCATCCTGAAGTCGCTCTCTCAAGTAGAAT $13 \mathrm{~A}$

GCGACGACCTGACGACATAGATCGGCTGCGAGCATGTAGAAACCTATCATATGTCG CTCTCTCAAGTAGAAT

13B GTCGCTCTCTCAAGTAGAATATCGGCTGCGAGCATG TAGAAACCTATCATAT 15A GCGACGACCTGACGACATAGGCGTTATAGAAAAAGCCTGTTTAGAAGGCCGG 15B GCGTTATAGAAAAAGC CTGTTTAG AAGGCCGGGCGACGACCTGACGACATAG 17

GCGACGACCTGACGACATAGAATTACTACAAATTCTTACCAGTAATCCCATCGTCG CTCTCTCAAGTAGAAT

19 TAGAATCCCTGAGAAGAGTCAATAGGAATCATGTCGCTCTCTCAAGTAGAAT 39 GGTATTAAGAACAAGAAAAATAATTAAAGCCAGTCGCTCTCTCAAGTAGAAT 41

GCGACGACCTGACGACATAGACGCTCAAAATAAGAATAAACACCGTGAATTTGTC GCTCTCTCAAGTAGAAT

43 GCGACGACCTGACGACATAGATCAAAATCGTCGCTATTAATTAACGGATTCG 42 GCGACGACCTGACGACATAGAGGCGTTACAGTAGGGCTTAATTGACAATAGA 44

GCGACGACCTGACGACATAGCTGTAAATCATAGGTCTGAGAGACGATAAATAGTCG CTCTCTCAAGTAGAAT 46 ACAGAAATCTTTGAATACCAAGTTCCTTGCTTGTCGCTCTCTCAAGTAGAAT 65 CATATTTAGAAATACCGACCGTGTTACCTTTTGTCGCTCTCTCAAGTAGAAT 67

GCGACGACCTGACGACATAGTAACCTCCATATGTGAGTGAATAAACAAAATCGTCG CTCTCTCAAGTAGAAT 69 GCGACGACCTGACGACATAGGCGCAGAGATATCAAAATTATTTGACATTATC 
68 GCGACGACCTGACGACATAGAAATCAATGGCTTAGGTTGGGTTACTAAATTT 70

GCGACGACCTGACGACATAGAACCTACCGCGAATTATTCATTTCCAGTACATGTCG CTCTCTCAAGTAGAAT

72 CTAAAATAGAACAAAGAAACCACCAGGGTTAGGTCGCTCTCTCAAGTAGAAT 91 TATGTAAACCTTTTTTAATGGAAAAATTACCTGTCGCTCTCTCAAGTAGAAT 93

GCGACGACCTGACGACATAGGAGCAAAAACTTCTGAATAATGGAAGAAGGAGGT CGCTCTCTCAAGTAGAAT

95 GCGACGACCTGACGACATAGCGGAATTATTGAAAGGAATTGAGGTGAAAAAT 94 GCGACGACCTGACGACATAGTGGATTATGAAGATGATGAAACAAAATTTCAT 96

GCGACGACCTGACGACATAGATCAACAGTCATCATATTCCTGATTGATTGTTGTCGC TCTCTCAAGTAGAAT

98 GCCAACAGTCACCTTGCTGAACCTGTTGGCAAGTCGCTCTCTCAAGTAGAAT 120

GTCGCTCTCTCAAGTAGAATAGGTAAAGAAATCACCATCAATATAATATTTTGCGAC GACCTGACGACATAG

118 GTCGCTCTCTCAAGTAGAATGTTAAAATTTTAACCAATAGGAACCCGGCACC 142 GTCGCTCTCTCAAGTAGAATACCGTTCTAAATGCAATGCCTGAGAGGTGGCA 140

GTCGCTCTCTCAAGTAGAATAAATAATTTTAAATTGTAAACGTTGATATTCAGCGAC GACCTGACGACATAG

138 GGCGATCGCACTCCAGCCAGCTTTGCCATCAAGCGACGACCTGACGACATAG 143 TATATTTTAGCTGATAAATTAATGTTGTATAAGCGACGACCTGACGACATAG 141

GTCGCTCTCTCAAGTAGAATGCAAATATCGCGTCTGGCCTTCCTGGCCTCAGGCGA CGACCTGACGACATAG

139 GTCGCTCTCTCAAGTAGAATGAAGATCGGTGCGGGCCTCTTCGCAATCATGG

164 GTCGCTCTCTCAAGTAGAATCTTTCATCCCCAAAAACAGGAAGACCGGAGAG 162

GTCGCTCTCTCAAGTAGAATCAGCTGGCGGACGACGACAGTATCGTAGCCAGGCG ACGACCTGACGACATAG

160 ACTGCCCGCCGAGCTCGAATTCGTTATTACGCGCGACGACCTGACGACATAG

165 AGAAAAGCAACATTAAATGTGAGCATCTGCCAGCGACGACCTGACGACATAG 163

GTCGCTCTCTCAAGTAGAATGTTTGAGGGAAAGGGGGATGTGCTAGAGGATCGCG ACGACCTGACGACATAG

161 GTCGCTCTCTCAAGTAGAATCCCGGGTACTTTCCAGTCGGGAAACGGGCAAC

186 GTCGCTCTCTCAAGTAGAATATTAAGTTCGCATCGTAACCGTGCGAGTAACA 184

GTCGCTCTCTCAAGTAGAATGCCAGCTGCCTGCAGGTCGACTCTGCAAGGCGGCG ACGACCTGACGACATAG

182 TGGACTCCCTTTTCACCAGTGAGACCTGTCGTGCGACGACCTGACGACATAG 
187 TAGATGGGGGGTAACGCCAGGGTTGTGCCAAGGCGACGACCTGACGACATAG 185

GTCGCTCTCTCAAGTAGAATCTTGCATGCATTAATGAATCGGCCCGCCAGGGGCGA CGACCTGACGACATAG

183 GTCGCTCTCTCAAGTAGAATTGGTTTTTAACGTCAAAGGGCGAAGAACCATC 9 AAAAGTAATATCTTACCGAAGCCCTTCCAGAGGCGACGACCTGACGACATAG 10 GTCGCTCTCTCAAGTAGAATGCAATAGCGCAGATAGCCGAACAATTCAACCG 12

GTCGCTCTCTCAAGTAGAATTCTTACCAGCCAGTTACAAAATAAATGAAATAGCGA CGACCTGACGACATAG

33 GACTTGAGAGACAAAAGGGCGACAAGTTACCAGCGACGACCTGACGACATAG 35

GTCGCTCTCTCAAGTAGAATGAAGGAAAATAAGAGCAAGAAACAACAGCCATGCG ACGACCTGACGACATAG

37 GTCGCTCTCTCAAGTAGAATATTATTTAACCCAGCTACAATTTTCAAGAACG 34 GTCGCTCTCTCAAGTAGAATAGCGCCAACCATTTGGGAATTAGATTATTAGC 36

GTCGCTCTCTCAAGTAGAATGCCCAATACCGAGGAAACGCAATAGGTTTACCGCGA CGACCTGACGACATAG

38 TATTTTGCTCCCAATCCAAATAAGTGAGTTAAGCGACGACCTGACGACATAG 55 CACCAGAGTTCGGTCATAGCCCCCGCCAGCAAGCGACGACCTGACGACATAG 57

GTCGCTCTCTCAAGTAGAATAATCACCAAATAGAAAATTCATATATAACGGAGCGA CGACCTGACGACATAG

59 GTCGCTCTCTCAAGTAGAATATACCCAAGATAACCCACAAGAATAAACGATT 56 GTCGCTCTCTCAAGTAGAATTCGGCATTCCGCCGCCAGCATTGACGTTCCAG 58

GTCGCTCTCTCAAGTAGAATTCACAATCGTAGCACCATTACCATCGTTTTCAGCGA CGACCTGACGACATAG

60 ATCAGAGAAAGAACTGGCATGATTTTATTTTGGCGACGACCTGACGACATAG 77 TGCTCAGTCAGTCTCTGAATTTACCAGGAGGTGCGACGACCTGACGACATAG 79

GTCGCTCTCTCAAGTAGAATTGAGGCAGGCGTCAGACTGTAGCGTAGCAAGGGCG ACGACCTGACGACATAG

81 GTCGCTCTCTCAAGTAGAATCCGGAAACACACCACGGAATAAGTAAGACTCC 78 GTCGCTCTCTCAAGTAGAATGGAAAGCGACCAGGCGGATAAGTGAATAGGTG 80

GTCGCTCTCTCAAGTAGAATTGCCTTTAGTCAGACGATTGGCCTGCCAGAATGCGA CGACCTGACGACATAG

82 ACGCAAAGGTCACCAATGAAACCAATCAAGTTGCGACGACCTGACGACATAG

\section{Elongated staples (50-nm pitch)}

203

GCGACGACCTGACGACATAGGAGAATAGCTTTTGCGGGATCGTCGGGTAGCAGCG 
ACGACCTGACGACATAG

201 GCGACGACCTGACGACATAGACGGCTACTTACTTAGCCGGAACGCTGACCAA 202 GCGACGACCTGACGACATAGAAAGGCCG AAAGGAACAACTAAAGCTTTCCAG 200

GCGACGACCTGACGACATAGGCTCCATGAGAGGCTTTGAGGACTAGGGAGTTGCG ACGACCTGACGACATAG

198

GCGACGACCTGACGACATAGCGATTTTAGAGGACAGATGAACGGCGCGACCTGCG ACGACCTGACGACATAG

196 ACTGGATA ACGGAACA ACATTATTACCTTATGGCGACGACCTGACGACATAG

177 TTTCATGAAAATTGTGTCGAAATCTGTACAGAGCGACGACCTGACGACATAG

175 GCGACGACCTGACGACATAGCCAGGCGCTTAATCATTGTGAATTACAGGTAG GCGACGACCTGACGACATAG

173 GCGACGACCTGACGACATAGAAAGATTCAGGGGGTAATAGTAAACCATAAAT GCGACGACCTGACGACATAG

171 GCGACGACCTGACGACATAGCAAAAATCATTGCTCCTTTTGATAAGTTTCAT 170

GCGACGACCTGACGACATAGTACCTTTAAGGTCTTTACCCTGACAAAGAAGTGCG ACGACCTGACGACATAG

168-I

GCGACGACCTGACGACATAGCAATAAATACAGTTGATTCCCAATTTAGAGAGGCGA CGACCTGACGACATAG

168-II GCGACGACCTGACGACATAGCAATAAATACAGTTGATTCCCAATTTAGAGAG 166-I GGTAGCTAGGATAAAAATTTTTAGTTAACATCGCGACGACCTGACGACATAG $166-\mathrm{II}$ GCGACGACCTGACGACATAGGGTAGCTAGGATAAAAATTTTTAGTTAACATCGCGA CGACCTGACGACATAG

147-I

TCAGAAGCCTCCAACAGGTCAGGATCTGCGAAGCGACGACCTGACGACATAG 147-II

GCGACGACCTGACGACATAGTCAGAAGCCTCCAACAGGTCAGGATCTGCGAA GCGACGACCTGACGACATAG

145

GCGACGACCTGACGACATAGCGAGTAGAACTAATAGTAGTAGCAAACCCTCAGCG ACGACCTGACGACATAG

143-I

GCGACGACCTGACGACATAGTATATTTTAGCTGATAAATTAATGTTGTATAAGCGAC GACCTGACGACATAG

143-II GCGACGACCTGACGACATAGTATATTTTAGCTGATAAATTAATGTTGTATAA 141 GCGACGACCTGACGACATAGGCAAATATCGCGTCTGGCCTTCCTGGCCTCAG 142 GCGACGACCTGACGACATAGACCGTTCTAAATGCAATGCCTGAGAGGTGGCA 140 GCGACGACCTGACGACATAGAAATAATTTTAAATTGTAAACGTTGATATTCA GCGACGACCTGACGACATAG 138 
GCGACGACCTGACGACATAGGGCGATCGCACTCCAGCCAGCTTTGCCATCAAGCG ACGACCTGACGACATAG

136 GTGAGCTAGTTTCCTGTGTGAAATTTGGGAAGGCGACGACCTGACGACATAG 118 GTTAAAATTTTAACCAATAGGAACCCGGCACCGCGACGACCTGACGACATAG 117 GCGACGACCTGACGACATAGGCTTCTGGTCAGGCTGCGCAACTGTGTTATCCGCG ACGACCTGACGACATAG

115

GCGACGACCTGACGACATAGGCTCACAATGTAAAGCCTGGGGTGGGTTTGCCGCG ACGACCTGACGACATAG

113 GCGACGACCTGACGACATAGCCAGCAGGGGCAAAATCCCTTATAAAGCCGGC 114

GCGACGACCTGACGACATAGGCATAAAGTTCCACACAACATACGAAGCGCCAGCG ACGACCTGACGACATAG

112

GCGACGACCTGACGACATAGCCGAAATCCGAAAATCCTGTTTGAAGCCGGAAGCG ACGACCTGACGACATAG

24-I ACGAACCAAAACATCGCCATTAAATGGTGGTTGCGACGACCTGACGACATAG

24-III GCGACGACCTGACGACATAGACGAACCAAAACATCGCCATTAAATGGTGGTT 22 CGACAACTAAGTATTAGACTTTACAATACCGAGCGACGACCTGACGACATAG 23

GCGACGACCTGACGACATAGGGATTTAGCGTATTAAATCCTTTGTTTTCAGGGCGA CGACCTGACGACATAG

26

GCGACGACCTGACGACATAGTAGCCCTACCAGCAGAAGATAAAAACATTTGAGCG ACGACCTGACGACATAG

41 ACGCTCAAAATAAGAATAAACACCGTGAATTTGCGACGACCTGACGACATAG

43

GCGACGACCTGACGACATAGATCAAAATCGTCGCTATTAATTAACGGATTCGGCGA CGACCTGACGACATAG

45

GCGACGACCTGACGACATAGCCTGATTGAAAGAAATTGCGTAGACCCGAACGGCG ACGACCTGACGACATAG

47 GCGACGACCTGACGACATAGTTATTAATGCCGTCAATAGATAATCAGAGGTG

40-III GCGACGACCTGACGACATAGTAAGTCCTACCAAGTACCGCACTCTTAGTTGC 40-IV

GCGACGACCTGACGACATAGTAAGTCCTACCAAGTACCGCACTCTTAGTTGCGCG ACGACCTGACGACATAG

42-III

GCGACGACCTGACGACATAGAGGCGTTACAGTAGGGCTTAATTGACAATAGAGCG ACGACCTGACGACATAG

42-IV AGGCGTTACAGTAGGGCTTAATTGACAATAGAGCGACGACCTGACGACATAG 44

GCGACGACCTGACGACATAGCTGTAAATCATAGGTCTGAGAGACGATAAATAGCG 
ACGACCTGACGACATAG

46 ACAGAAATCTTTGAATACCAAGTTCCTTGCTTGCGACGACCTGACGACATAG

59 ATACCCAAGATAACCC ACAAGAATAAACGATTGCGACGACCTGACGACATAG

61-III

GCGACGACCTGACGACATAGTTTTGTTTAAGCCTTAAATCAAGAATCGAGAAGCG ACGACCTGACGACATAG

61-IV TTTTGTTTAAGCCTTAAATCAAGAATCGAGAAGCGACGACCTGACGACATAG

63

GCGACGACCTGACGACATAGCAAGCAAGACGCGCCTGTTTATCAAGAATCGCGCG ACGACCTGACGACATAG

65-III GCGACGACCTGACGACATAGCATATTTAGAAATACCGACCGTGTTACCTTTT

65-IV GCGACGACCTGACGACATAGCATATTTAGAAATACCGACCGTGTTACCTTTT GCGACGACCTGACGACATAG

58 GCGACGACCTGACGACATAGTCACAATCGTAGCACCATTACCATCGTTTTCA

60

GCGACGACCTGACGACATAGATCAGAGAAAGAACTGGCATGATTTTATTTTGGCGA CGACCTGACGACATAG

62

GCGACGACCTGACGACATAGAGGTTTTGAACGTCAAAAATGAAAGCGCTAATGCG ACGACCTGACGACATAG

64 AATGCAGACCGTTTTTATTTTCATCTTGCGGGGCGACGACCTGACGACATAG

77 TGCTCAGTCAGTCTCT GAATTTACCAGGAGGTGCGACGACCTGACGACATAG

79

GCGACGACCTGACGACATAGTGAGGCAGGCGTCAGACTGTAGCGTAGCAAGGGC

GACGACCTGACGACATAG

81

GCGACGACCTGACGACATAGCCGGAAACACACCACGGAATAAGTAAGACTCCGC GACGACCTGACGACATAG

83 GCGACGACCTGACGACATAGTTATTACGGTCAGAGGGTAATTGAATAGCAGC 78

GCGACGACCTGACGACATAGGGAAAGCGACCAGGCGGATAAGTGAATAGGTGGC GACGACCTGACGACATAG

80

GCGACGACCTGACGACATAGTGCCTTTAGTCAGACGATTGGCCTGCCAGAATGCG ACGACCTGACGACATAG

82

ACGCAAAGGTCACCAATGAAACCAATCAAGTTGCGACGACCTGACGACATAG

2

GCGACGACCTGACGACATAGAATGCCCCGTAACAGTGCCCGTATCTCCCTCAGCG

ACGACCTGACGACATAG

3 GCGACGACCTGACGACATAGTGCCTTGACTGCCTAT TTCGGAACAGGGATAG

4 GCGACGACCTGACGACATAGGAGCCGCCCCACCACCGGAACCGCGACGGAAA

5

GCGACGACCTGACGACATAGAACCAGAGACCCTCAGAACCGCCAGGGGTCAGGC 
GACGACCTGACGACATAG

8

GCGACGACCTGACGACATAGATTGAGGGTAAAGGTGAATTATCAATCACCGGGCG ACGACCTGACGACATAG

10 GCAATAGCGCAGATAG CCGAACAATTCAACCGGCGACGACCTGACGACATAG

31 GCCACCACTCTTTTCA TAATCAAACCGTCACCGCGACGACCTGACGACATAG

33

GCGACGACCTGACGACATAGGACTTGAGAGACAAAAGGGCGACAAGTTACCAGC GACGACCTGACGACATAG

35

GCGACGACCTGACGACATAGGAAGGAAAATAAGAGCAAGAAACAACAGCCATGC GACGACCTGACGACATAG

37 GCGACGACCTGACGACATAGATTATTTA ACCCAGCTACAATTTTCAAGAACG 36 GCGACGACCTGACGACATAGGCCCAATA CCGAGGAA ACGCAATA GGTTTACC 38

GCGACGACCTGACGACATAGTATTTTGCTCCCAATCCAAATAAGTGAGTTAAGCGA CGACCTGACGACATAG

67 GCGACGACCTGACGACATAGTAACCTCCATATGTGAGTGAATAAACAAAATC 66 GCGACGACCTGACGACATAGAATGGTTTACAACGCCAACATGTAGTTCAGCT 68

GCGACGACCTGACGACATAGAAATCAATGGCTTAGGTTGGGTTACTAAATTTGCGA CGACCTGACGACATAG

70

GCGACGACCTGACGACATAGAACCTACCGCGAATTATTCATTTCCAGTACATGCGA CGACCTGACGACATAG

72 CTAAAATAGAACAAAGAAACCACCAGGGTTAGGCGACGACCTGACGACATAG

91 TATGTAAACCTTTTTTAATGGAAAAATTACCTGCGACGACCTGACGACATAG

93

GCGACGACCTGACGACATAGGAGCAAAAACTTCTGAATAATGGAAGAAGGAGGC GACGACCTGACGACATAG

95

GCGACGACCTGACGACATAGCGGAATTATTGAAAGGAATTGAGGTGAAAAATGCG ACGACCTGACGACATAG

97 GCGACGACCTGACGACATAGCTAAAGCAAGATAGAACCCTTCTGAATCGTCT 96 GCGACGACCTGACGACATAGATCAACAGTCATCATATTCCTGATTGATTGTT 98

GCGACGACCTGACGACATAGGCCAACAGTCACCTTGCTGAACCTGTTGGCAAGCG ACGACCTGACGACATAG

130

GCGACGACCTGACGACATAGCTTAAACATCAGCTTGCTTTCGAGCGTAACACGCG ACGACCTGACGACATAG

128

GCGACGACCTGACGACATAGCTCATCTTGAGGCAAAAGAATACAGTGAATTTGCG ACGACCTGACGACATAG 
126 GAATAAGGACGTAACAAAGCTGCTCTAAAACAGCGACGACCTGACGACATAG

131 TCGGTTTAGCTTGATACCGATAGTCCAACCTAGCGACGACCTGACGACATAG 129

GCGACGACCTGACGACATAGAAACGAAATGACCCCCAGCGATTATTCATTACGCG ACGACCTGACGACATAG

127

GCGACGACCTGACGACATAGCCAAATCACTTGCCCTGACGAGAACGCCAAAAGC GACGACCTGACGACATAG

125 GCGACGACCTGACGACATAGGGAATTACTCGTTTACCAGACGACAAAAGATT

150 GCGACGACCTGACGACATAGACGAGTAGTGACAAGAACCGGATATACCAAGC 148 GCGACGACCTGACGACATAGCCAAAATATAATGCAGATACATAAACACCAGA GCGACGACCTGACGACATAG

146 GCGACGACCTGACGACATAGGAAGCAAAAAAGCGGATTGCATCAGATAAAAA GCGACGACCTGACGACATAG

144 TCAATTCTTTTAGTTTGACCATTACCAGACCGGCGACGACCTGACGACATAG 149 CATTCAACGCGAGAGGCTTTTGCATATTATAGGCGACGACCTGACGACATAG 164

GCGACGACCTGACGACATAGCTTTCATCCCCAAAAACAGGAAGACCGGAGAGGC GACGACCTGACGACATAG

162 CAGCTGGCGGACGACGACAGTATCGTAGCCAGGCGACGACCTGACGACATAG 167 CAACGCAATTTTTGAGAGATCTACTGATAATCGCGACGACCTGACGACATAG 165

GCGACGACCTGACGACATAGAGAAAAGCAACATTAAATGTGAGCATCTGCCAGCG ACGACCTGACGACATAG

163

GCGACGACCTGACGACATAGGTTTGAGGGAAAGGGGGATGTGCTAGAGGATCGCG ACGACCTGACGACATAG

161 GCGACGACCTGACGACATAGCCCGGGTACTTTCCAGTCGGGAAACGGGCAAC 186 GCGACGACCTGACGACATAGATTAAGTTCGCATCGTAACCGTGCGAGTAACA 184

GCGACGACCTGACGACATAGGCCAGCTGCCTGCAGGTCGACTCTGCAAGGCGGC GACGACCTGACGACATAG

182

GCGACGACCTGACGACATAGTGGACTCCCTTTTCACCAGTGAGACCTGTCGTGCG ACGACCTGACGACATAG 185 CTTGCATGCATTAATGAATCGGCCCGCCAGGGGCGACGACCTGACGACATAG 183

GCGACGACCTGACGACATAGTGGTTTTTAACGTCAAAGGGCGAAGAACCATCGCG ACGACCTGACGACATAG

For bifacial pcDNA templates on rectangular DNA origami

184 GCGACGACCTGACGACATAGGCCAGCTGCCTGCAGGTCGACTCTGCAAGGCG 185 GCGACGACCTGACGACATAGCTTGCATGCATTAATG 185\# GCGACGACCTGACGACATAGAATCGGCCCGCCAGGG 
186 GCGACGACCTGACGACATAGATTAAGTTCGCATCGTAACCGTGCGAGTAACA 187 GCGACGACCTGACGACATAGTAGATGGGGGGTAACGCCAGGGTTGTGCCAAG 188 GCGACGACCTGACGACATAGACCCGTCGTCATATGTACCCCGGTAAAGGCTA 189 GCGACGACCTGACGACATAGCATGTCAAGATTCTCCGTGGGAACCGTTGGTG 190 GCGACGACCTGACGACATAGTCAGGTCACTTTTGCGGGAGAAGCAGAATTAG 191 GCGACGACCTGACGACATAGCTGTAATATTGCCTGAGAGTCTGGAAAACTAG 192 GCGACGACCTGACGACATAGCAAAATTAAAGTACGGTGTCTGGAAGAGGTCA 193 GCGACGACCTGACGACATAGTGCAACTAAGCAATAAAGCCTCAGTTATGACC 194 GCGACGACCTGACGACATAGTTTTTGCGCAGAAAACGAGAATGAATGTTTAG 195 GCGACGACCTGACGACATAGAAACAGTTGATGGCTTAGAGCTTATTTAAATA 196 GCGACGACCTGACGACATAGACTGGATAACGGAACAACATTATTACCTTATG 197 GCGACGACCTGACGACATAGACGAACTAGCGTCCAATACTGCGGAATGCTTT 198 GCGACGACCTGACGACATAGCGATTTTAGAGGACAGATGAACGGCGCGACCT 199 GCGACGACCTGACGACATAGCTTTGAAAAGAACTGGCTCATTATTTAATAAA 200 GCGACGACCTGACGACATAGGCTCCATGAGAGGCTTTGAGGACTAGGGAGTT 201 GCGACGACCTGACGACATAGACGGCTACTTACTTAGCCGGAACGCTGACCAA 202 GCGACGACCTGACGACATAGAAAGGCCGAAAGGAACAACTAAAGCTTTCCAG 203 GCGACGACCTGACGACATAGGAGAATAGCTTTTGCGGGATCGTCGGGTAGCA 204 GCGACGACCTGACGACATAGACGTTAGTAAATGAATTTTCTGTAAGCGGAGT 181 ACCCAAATCAAGTTTT

181\# GCGACGACCTGACGACATAGTTGGGGTCAAAGAACG 182 TGGACTCCCTTTTCAC 182\# GCGACGACCTGACGACATAGCAGTGAGACCTGTCGT 183 GCGACGACCTGACGACATAGTGGTTTTTAACGTCAA 183\# GCGACGACCTGACGACATAGAGGGCGAAGAACCATC 76 GCGACGACCTGACGACATAGTATCACCGTACTCAGGAGGTTTAGCGGGGTTT 77 GCGACGACCTGACGACATAGTGCTCAGTCAGTCTCTGAATTTACCAGGAGGT 78 GCGACGACCTGACGACATAGGGAAAGCGACCAGGCGGATAAGTGAATAGGTG 79 GCGACGACCTGACGACATAGTGAGGCAGGCGTCAGACTGTAGCGTAGCAAGG 80 GCGACGACCTGACGACATAGTGCCTTTAGTCAGACGATTGGCCTGCCAGAAT 81 GCGACGACCTGACGACATAGCCGGAAACACACCACGGAATAAGTAAGACTCC 82 GCGACGACCTGACGACATAGACGCAAAGGTCACCAATGAAACCAATCAAGTT 83 GCGACGACCTGACGACATAGTTATTACGGTCAGAGGGTAATTGAATAGCAGC 84 GCGACGACCTGACGACATAGTGAACAAACAGTATGTTAGCAAACTAAAAGAA 85 GCGACGACCTGACGACATAGCTTTACAGTTAGCGAACCTCCCGACGTAGGAA 86 GCGACGACCTGACGACATAGGAGGCGTTAGAGAATAACATAAAAGAACACCC 87 GCGACGACCTGACGACATAGTCATTACCCGACAATAAACAACATATTTAGGC 88 GCGACGACCTGACGACATAGCCAGACGAGCGCCCAATAGCAAGCAAGAACGC 89 GCGACGACCTGACGACATAGAGAGGCATAATTTCATCTTCTGACTATAACTA 90 GCGACGACCTGACGACATAGTTTTAGTTTTTCGAGCCAGTAATAAATTCTGT 91 GCGACGACCTGACGACATAGTATGTAAACCTTTTTTAATGGAAAAATTACCT 92 GCGACGACCTGACGACATAGTTGAATTATGCTGATGCAAATCCACAAATATA 93 GCGACGACCTGACGACATAGGAGCAAAAACTTCTGAATAATGGAAGAAGGAG 94 GCGACGACCTGACGACATAGTGGATTATGAAGATGATGAAACAAAATTTCAT 
95 GCGACGACCTGACGACATAGCGGAATTATTGAAAGG

95\# GCGACGACCTGACGACATAGAATTGAGGTGAAAAAT

96 GCGACGACCTGACGACATAGATCAACAGTCATCATATTCCTGATTGATTGTT

97 GCGACGACCTGACGACATAGCTAAAGCAAGATAGAA

97\# GCGACGACCTGACGACATAGCCCTTCTGAATCGTCT

98 GCCAACAGTCACCTTG

98\# GCGACGACCTGACGACATAGCTGAACCTGTTGGCAA

99 GAAATGGATTATTTAC

99\# GCGACGACCTGACGACATAGATTGGCAGACATTCTG

2 GCGACGACCTGACGACATAGAATGCCCCGTAACAGTGCCCGTATCTCCCTCA

4 GCGACGACCTGACGACATAGGAGCCGCCCCACCACCGGAACCGCGACGGAAA

6 GCGACGACCTGACGACATAGTTATTCATAGGGAAGGTAAATATTCATTCAGT

9 GCGACGACCTGACGACATAGAAAAGTAATATCTTACCGAAGCCCTTCCAGAG

11 GCGACGACCTGACGACATAGCCTAATTTACGCTAACGAGCGTCTAATCAATA

13 GCGACGACCTGACGACATAGATCGGCTGCGAGCATGTAGAAACCTATCATAT

15 GCGACGACCTGACGACATAGGCGTTATAGAAAAAGCCTGTTTAGAAGGCCGG

16 GCGACGACCTGACGACATAGGCTCATTTTCGCATTAAATTTTTGAGCTTAGA

18 GCGACGACCTGACGACATAGTTAAGACGTTGAAAACATAGCGATAACAGTAC 116 GCGACGACCTGACGACATAGTTCGCCATTGCCGGAAACCAGGCATTAAATCA 20 GCGACGACCTGACGACATAGCTTTTACACAGATGAATATACAGTAAACAATT

114 GCGACGACCTGACGACATAGGCATAAAGTTCCACACAACATACGAAGCGCCA 71 ATTTTGCGTCTTTAGG

71\# GCGACGACCTGACGACATAGAGCACTAAGCAACAGT

74 GCGTAAGAGAGAGCCA

74\# GCGACGACCTGACGACATAGGCAGCAAAAAGGTTAT

73 GCCACGCTATACGTGG

73\# GCGACGACCTGACGACATAGCACAGACAACGCTCAT 75 GGAAATACCTACATTT

75\# GCGACGACCTGACGACATAGTGACGCTCACCTGAAA 47 TTATTAATGCCGTCAA

47\# GCGACGACCTGACGACATAGTAGATAATCAGAGGTG 50 GAATGGCTAGTATTAA

50\# GCGACGACCTGACGACATAGCACCGCCTCAACTAAT

49 AGGCGGTCATTAGTCT

49\# GCGACGACCTGACGACATAGTTAATGCGCAATATTA

51 CCGCCAGCCATTGCAA

51\# GCGACGACCTGACGACATAGCAGGAAAAATATTTTT

22 CGACAACTAAGTATTA

22\# GCGACGACCTGACGACATAGGACTTTACAATACCGA

26 TAGCCCTACCAGCAGA

26\# GCGACGACCTGACGACATAGAGATAAAAACATTTGA

27 CGGCCTTGCTGGTAAT

27\# GCGACGACCTGACGACATAGATCCAGAACGAACTGA

157 GTAAAGCACTAAATCG 
157\# GCGACGACCTGACGACATAGGAACCCTAGTTGTTCC

159 AGCTGATTACAAGAGT

159\# GCGACGACCTGACGACATAGCCACTATTGAGGTGCC

158 AGTTTGGAGCCCTTCA

158\# GCGACGACCTGACGACATAGCCGCCTGGTTGCGCTC

161 CCCGGGTACTTTCCAG

161\# GCGACGACCTGACGACATAGTCGGGAAACGGGCAAC

133 CCCCGATTTAGAGCTT

133\# GCGACGACCTGACGACATAGGACGGGGAAATCAAAA

135 GAGTTGCACGAGATAG

135\# GCGACGACCTGACGACATAGGGTTGAGTAAGGGAGC

134 GAATAGCCGCAAGCGG

134\# GCGACGACCTGACGACATAGTCCACGCTCCTAATGA

137 TCATAGCTACTCACAT

137\# GCGACGACCTGACGACATAGTAATTGCGCCCTGAGA

113 CCAGCAGGGGCAAAAT

113\# GCGACGACCTGACGACATAGCCCTTATAAAGCCGGC

112 CCGAAATCCGAAAATC

112\# GCGACGACCTGACGACATAGCTGTTTGAAGCCGGAA

115 GCTCACAATGTAAAGC

115\# GCGACGACCTGACGACATAGCTGGGGTG GGTTTGCC

\# staples are used to create non-coplanar protrusion

For the bilateral pcDNA template on triangular DNA origami

A01 CGGGGTTTCCTCAAGAGAAGGATTTTGAATTAGCGACGACCTGACGACATAG

A02 AGCGTCATGTCTCTGAATTTACCGACTACCTTGCGACGACCTGACGACATAG

A03 TTCATAATCCCCTTATTAGCGTTTTTCTTACCGCGACGACCTGACGACATAG

A04 ATGGTTTATGTCACAATCAATAGATATTAAACGCGACGACCTGACGACATAG

A05

TTTGATGATTAAGAGGCTGAGACTTGCTCAGTACCAGGCGGCGACGACCTGACGA CATAG

A06 CCGGAACCCAGAATGGAAAGCGCAACATGGCTGCGACGACCTGACGACATAG A07 AAAGACAACATTTTCGGTCATAGCCAAAATCAGCGACGACCTGACGACATAG A08

GACGGGAGAATTAACTCGGAATAAGTTTATTTCCAGCGCCGCGACGACCTGACGA CATAG

A09

GATAAGTGCCGTCGAGCTGAAACATGAAAGTATACAGGAGGCGACGACCTGACGA CATAG

A10 TGTACTGGAAATCCTCATTAAAGCAGAGCCACGCGACGACCTGACGACATAG

A11 CACCGGAAAGCGCGTTTTCATCGGAAGGGCGAGCGACGACCTGACGACATAG

A12

CATTCAACAAACGCAAAGACACCAGAACACCCTGAACAAAGCGACGACCTGACG ACATAG 
A13

TTTAACGGTTCGGAACCTATTATTAGGGTTGATATAAGTAGCGACGACCTGACGAC ATAG

A14 CTCAGAGCATATTCACAAACAAATTAATAAGTGCGACGACCTGACGACATAG

A15 GGAGGGAATTTAGCGTCAGACTGTCCGCCTCCGCGACGACCTGACGACATAG A16

GTCAGAGGGTAATTGATGGCAACATATAAAAGCGATTGAGGCGACGACCTGACGA CATAG

A17

TAGCCCGGAATAGGTGAATGCCCCCTGCCTATGGTCAGTGGCGACGACCTGACGA CATAG

A18 CCTTGAGTCAGACGATTGGCCTTGCGCCACCCGCGACGACCTGACGACATAG

A19 TCAGAACCCAGAATCAAGTTTGCCGGTAAATAGCGACGACCTGACGACATAG

A20

TTGACGGAAATACATACATAAAGGGCGCTAATATCAGAGAGCGACGACCTGACGA CATAG

A21

CAGAGCCAGGAGGTTGAGGCAGGTAACAGTGCCCGGCGACGACCTGACGACATA $\mathrm{G}$

A22 ATTAAAGGCCGTAATCAGTAGCGAGCCACCCTGCGACGACCTGACGACATAG

A23

GATAACCCACAAGAATGTTAGCAAACGTAGAAAATTATTCGCGACGACCTGACGA CATAG

A24 GCCGCCAGCATTGACACCACCCTCGCGACGACCTGACGACATAG

A25 AGAGCCGCACCATCGATAGCAGCATGAATTATGCGACGACCTGACGACATAG

A26

CACCGTCACCTTATTACGCAGTATTGAGTTAAGCCCAATAGCGACGACCTGACGAC ATAG

A27

AGCCATTTAAACGTCACCAATGAACACCAGAACCAGCGACGACCTGACGACATAG A28

ATAAGAGCAAGAAACATGGCATGATTAAGACTCCGACTTGGCGACGACCTGACGA CATAG

A29 CCATTAGCAAGGCCGGGGGAATTAGCGACGACCTGACGACATAG

A30

GAGCCAGCGAATACCCAAAAGAACATGAAATAGCAATAGCGCGACGACCTGACGA CATAG

A31

TATCTTACCGAAGCCCAAACGCAATAATAACGAAAATCACCAGGCGACGACCTGA CGACATAG

A32

CAGAAGGAAACCGAGGTTTTTAAGAAAAGTAAGCAGATAGCCGGCGACGACCTG ACGACATAG

A33 CCTTTTTTCATTTAACAATTTCATAGGATTAGGCGACGACCTGACGACATAG 
A34 TTTAACCTATCATAGGTCTGAGAGTTCCAGTAGCGACGACCTGACGACATAG A35 AGTATAAAATATGCGTTATACAAAGCCATCTTGCGACGACCTGACGACATAG A36 CAAGTACCTCATTCCAAGAACGGGAAATTCATGCGACGACCTGACGACATAG A37 AGAGAATAACATAAAAACAGGGAAGCGCATTAGCGACGACCTGACGACATAG A38

AAAACAAAATTAATTAAATGGAAACAGTACATTAGTGAATGCGACGACCTGACGA CATAG

A39 TTATCAAACCGGCTTAGGTTGGGTAAGCCTGTGCGACGACCTGACGACATAG A40 TTAGTATCGCCAACGCTCAACAGTCGGCTGTCGCGACGACCTGACGACATAG A41

TTTCCTTAGCACTCATCGAGAACAATAGCAGCCTTTACAGGCGACGACCTGACGAC ATAG

A42

AGAGTCAAAAATCAATATATGTGATGAAACAAACATCAAGGCGACGACCTGACGA CATAG

A43 ACTAGAAATATATAACTATATGTACGCTGAGAGCGACGACCTGACGACATAG A44 TCAATAATAGGGCTTAATTGAGAATCATAATTGCGACGACCTGACGACATAG A45

AACGTCAAAAATGAAAAGCAAGCCGTTTTTATGAAACCAAGCGACGACCTGACGA CATAG

A46

GAGCAAAAGAAGATGAGTGAATAACCTTGCTTATAGCTTAGCGACGACCTGACGA CATAG

A47 GATTAAGAAATGCTGATGCAAATCAGAATAAAGCGACGACCTGACGACATAG A48 CACCGGAATCGCCATATTTAACAAAATTTACGGCGACGACCTGACGACATAG A49 AGCATGTATTTCATCGTAGGAATCAAACGATTTTTTGTTTGCGACGACCTGACGAC ATAG

A50

ACATAGCGCTGTAAATCGTCGCTATTCATTTCAATTACCTGCGACGACCTGACGACA TAG

A51 GTTAAATACAATCGCAAGACAAAGCCTTGAAAGCGACGACCTGACGACATAG A52 CCCATCCTCGCCAACATGTAATTTAATAAGGCGCGACGACCTGACGACATAG A53

TCCCAATCCAAATAAGATTACCGCGCCCAATAAATAATATGCGACGACCTGACGAC ATAG

A54

TCCCTTAGAATAACGCGAGAAAACTTTTACCGACCGCGACGACCTGACGACATAG A55 GTGTGATAAGGCAGAGGCATTTTCAGTCCTGAGCGACGACCTGACGACATAG A56

ACAAGAAAGCAAGCAAATCAGATAACAGCCATATTATTTAGCGACGACCTGACGA CATAG

A57 GTTTGAAATTCAAATATATTTTAGGCGACGACCTGACGACATAG A58 AATAGATAGAGCCAGTAATAAGAGATTTAATGGCGACGACCTGACGACATAG 
A59

GCCAGTTACAAAATAATAGAAGGCTTATCCGGTTATCAACGCGACGACCTGACGAC ATAG

A60

TTCTGACCTAAAATATAAAGTACCGACTGCAGAACGCGACGACCTGACGACATAG A61

GCGCCTGTTATTCTAAGAACGCGATTCCAGAGCCTAATTTGCGACGACCTGACGAC ATAG

A62 TCAGCTAAAAAAGGTAAAGTAATTGCGACGACCTGACGACATAG

A63

ACGCTAACGAGCGTCTGGCGTTTTAGCGAACCCAACATGTGCGACGACCTGACGA CATAG

A64

ACGACAATAAATCCCGACTTGCGGGAGATCCTGAATCTTACCAGCGACGACCTGA CGACATAG

A65

TGCTATTTTGCACCCAGCTACAATTTTGTTTTGAAGCCTTAAAGCGACGACCTGAC GACATAG

C01 TCGGGAGATATACAGTAACAGTACAAATAATTGCGACGACCTGACGACATAG C02 CCTGATTAAAGGAGCGGAATTATCTCGGCCTCGCGACGACCTGACGACATAG C03 GCAAATCACCTCAATCAATATCTGCAGGTCGAGCGACGACCTGACGACATAG C04 CGACCAGTACATTGGCAGATTCACCTGATTGCGCGACGACCTGACGACATAG $\mathrm{C} 05$

TGGCAATTTTTAACGTCAGATGAAAACAATAACGGATTCGGCGACGACCTGACGA CATAG

C06 AAGGAATTACAAAGAAACCACCAGTCAGATGAGCGACGACCTGACGACATAG C07 GGACATTCACCTCAAATATCAAACACAGTTGAGCGACGACCTGACGACATAG $\mathrm{C} 08$

TTGACGAGCACGTATACTGAAATGGATTATTTAATAAAAGGCGACGACCTGACGAC ATAG

C09

CCTGATTGCTTTGAATTGCGTAGATTTTCAGGCATCAATAGCGACGACCTGACGAC ATAG

C10 TAATCCTGATTATCATTTTGCGGAGAGGAAGGGCGACGACCTGACGACATAG

C11 TTATCTAAAGCATCACCTTGCTGATGGCCAACGCGACGACCTGACGACATAG

$\mathrm{C} 12$

AGAGATAGTTTGACGCTCAATCGTACGTGCTTTCCTCGTTGCGACGACCTGACGAC ATAG

C13

GATTATACACAGAAATAAAGAAATACCAAGTTACAAAATCGCGACGACCTGACGA CATAG

C14 TAGGAGCATAAAAGTTTGAGTAACATTGTTTGGCGACGACCTGACGACATAG

C15 TGACCTGACAAATGAAAAATCTAAAATATCTTGCGACGACCTGACGACATAG $\mathrm{C} 16$ 
AGAATCAGAGCGGGAGATGGAAATACCTACATAACCCTTCGCGACGACCTGACGA CATAG

$\mathrm{C} 17$

GCGCAGAGGCGAATTAATTATTTGCACGTAAATTCTGAATGCGACGACCTGACGAC ATAG

C18 AATGGAAGCGAACGTTATTAATTTCTAACAACGCGACGACCTGACGACATAG

C19 TAATAGATCGCTGAGAGCCAGCAGAAGCGTAAGCGACGACCTGACGACATAG

C20

GAATACGTAACAGGAAAAACGCTCCTAAACAGGAGGCCGAGCGACGACCTGACG ACATAG

C21

TCAATAGATATTAAATCCTTTGCCGGTTAGAACCTGCGACGACCTGACGACATAG

C22 CAATATTTGCCTGCAACAGTGCCATAGAGCCGGCGACGACCTGACGACATAG

$\mathrm{C} 23$

TTAAAGGGATTTTAGATACCGCCAGCCATTGCGGCACAGAGCGACGACCTGACGA

CATAG

C24 ACAATTCGACAACTCGTAATACATGCGACGACCTGACGACATAG

C25 TTGAGGATGGTCAGTATTAACACCTTGAATGGGCGACGACCTGACGACATAG

$\mathrm{C} 26$

CTATTAGTATATCCAGAACAATATCAGGAACGGTACGCCAGCGACGACCTGACGAC ATAG

$\mathrm{C} 27$

CGCGAACTAAAACAGAGGTGAGGCTTAGAAGTATTGCGACGACCTGACGACATAG $\mathrm{C} 28$

GAATCCTGAGAAGTGTATCGGCCTTGCTGGTACTTTAATGGCGACGACCTGACGAC ATAG

C29 ACCACCAGCAGAAGATGATAGCCCGCGACGACCTGACGACATAG

C30

TAAAACATTAGAAGAACTCAAACTTTTTATAATCAGTGAGGCGACGACCTGACGAC ATAG

C31

GCCACCGAGTAAAAGAACATCACTTGCCTGAGCGCCATTAAAAGCGACGACCTGA CGACATAG

$\mathrm{C} 32$

TCTTTGATTAGTAATAGTCTGTCCATCACGCAAATTAACCGTTGCGACGACCTGACG ACATAG

C33 CGCGTCTGATAGGAACGCCATCAACTTTTACAGCGACGACCTGACGACATAG

C34 AGGAAGATGGGGACGACGACAGTAATCATATTGCGACGACCTGACGACATAG

C35 CTCTAGAGCAAGCTTGCATGCCTGGTCAGTTGGCGACGACCTGACGACATAG

C36 CCTTCACCGTGAGACGGGCAACAGCAGTCACAGCGACGACCTGACGACATAG

C37 CGAGAAAGGAAGGGAAGCGTACTATGGTTGCTGCGACGACCTGACGACATAG

$\mathrm{C} 38$

GCTCATTTTTTAACCAGCCTTCCTGTAGCCAGGCATCTGCGCGACGACCTGACGAC ATAG 
C39 CAGTTTGACGCACTCCAGCCAGCTAAACGACGGCGACGACCTGACGACATAG C40 GCCAGTGCGATCCCCGGGTACCGAGTTTTTCTGCGACGACCTGACGACATAG $\mathrm{C} 41$ TTTCACCAGCCTGGCCCTGAGAGAAAGCCGGCGAACGTGGGCGACGACCTGACG ACATAG

C42

GTAACCGTCTTTCATCAACATTAAAATTTTTGTTAAATCAGCGACGACCTGACGAC ATAG

C43 ACGTTGTATTCCGGCACCGCTTCTGGCGCATCGCGACGACCTGACGACATAG C44 CCAGGGTGGCTCGAATTCGTAATCCAGTCACGGCGACGACCTGACGACATAG $\mathrm{C} 45$

TAGAGCTTGACGGGGAGTTGCAGCAAGCGGTCATTGGGCGGCGACGACCTGACG ACATAG

C46

GTTAAAATTCGCATTAATGTGAGCGAGTAACACACGTTGGGCGACGACCTGACGA CATAG

C47 TGTAGATGGGTGCCGGAAACCAGGAACGCCAGGCGACGACCTGACGACATAG C48 GGTTTTCCATGGTCATAGCTGTTTGAGAGGCGGCGACGACCTGACGACATAG

C49

GTTTGCGTCACGCTGGTTTGCCCCAAGGGAGCCCCCGATTGCGACGACCTGACGA CATAG

C50

GGATAGGTACCCGTCGGATTCTCCTAAACGTTAATATTTTGCGACGACCTGACGAC ATAG

C51 AGTTGGGTCAAAGCGCCATTCGCCCCGTAATGGCGACGACCTGACGACATAG C52 CGCGCGGGCCTGTGTGAAATTGTTGGCGATTAGCGACGACCTGACGACATAG C53

CTAAATCGGAACCCTAAGCAGGCGAAAATCCTTCGGCCAAGCGACGACCTGACGA CATAG

$\mathrm{C} 54$

CGGCGGATTGAATTCAGGCTGCGCAACGGGGGATGGCGACGACCTGACGACATAG C55 TGCTGCAAATCCGCTCACAATTCCCAGCTGCAGCGACGACCTGACGACATAG C56

TTAATGAAGTTTGATGGTGGTTCCGAGGTGCCGTAAAGCAGCGACGACCTGACGA CATAG

C57 TGGCGAAATGTTGGGAAGGGCGATGCGACGACCTGACGACATAG

C58 TGTCGTGCACACAACATACGAGCCACGCCAGCGCGACGACCTGACGACATAG C59

CAAGTTTTTTGGGGTCGAAATCGGCAAAATCCGGGAAACCGCGACGACCTGACGA CATAG

C60

TCTTCGCTATTGGAAGCATAAAGTGTATGCCCGCTGCGACGACCTGACGACATAG C61

TTCCAGTCCTTATAAATCAAAAGAGAACCATCACCCAAATGCGACGACCTGACGA 
CATAG

C62 GCGCTCACAAGCCTGGGGTGCCTAGCGACGACCTGACGACATAG

C63

CGATGGCCCACTACGTATAGCCCGAGATAGGGATTGCGTTGCGACGACCTGACGAC ATAG

C64

AACTCACATTATTGAGTGTTGTTCCAGAAACCGTCTATCAGGGGCGACGACCTGAC

GACATAG

C65

ACGTGGACTCCAACGTCAAAGGGCGAATTTGGAACAAGAGTCCGCGACGACCTG ACGACATAG

10-nt protrusion: GCGACGACCT

15-nt protrusion: GCGACGACCTGACGA

30-nt protrusion: GCGACGACCTGACGACATAGCCTATGTCAC

\section{REFERENCES}

1. Barone, V.; Cossi, M., Quantum Calculation of Molecular Energies and Energy Gradients in Solution by a Conductor Solvent Model. J. Phys. Chem. A 1998, 102 (11), 1995-2001.

2. Check, C. E.; Faust, T. O.; Bailey, J. M.; Wright, B. J.; Gilbert, T. M.; Sunderlin, L. S., Addition of Polarization and Diffuse Functions to the LANL2DZ Basis Set for P-Block Elements. J. Phys. Chem. A 2001, 105 (34), 8111-8116.

3. Weigend, F.; Ahlrichs, R., Balanced Basis Sets of Split Valence, Triple Zeta Valence and Quadruple Zeta Valence Quality for H to Rn: Design and Assessment of Accuracy. Phys. Chem. Chem. Phys. 2005, 7 (18), 3297-3305. 